\title{
Standard Model results from ATLAS
}

Toshi SUMIDA (CERN) on behalf of the ATLAS collaboration

27th June - I st July 20 | |

Hadron Structure 'I I

Tatranská Štrba

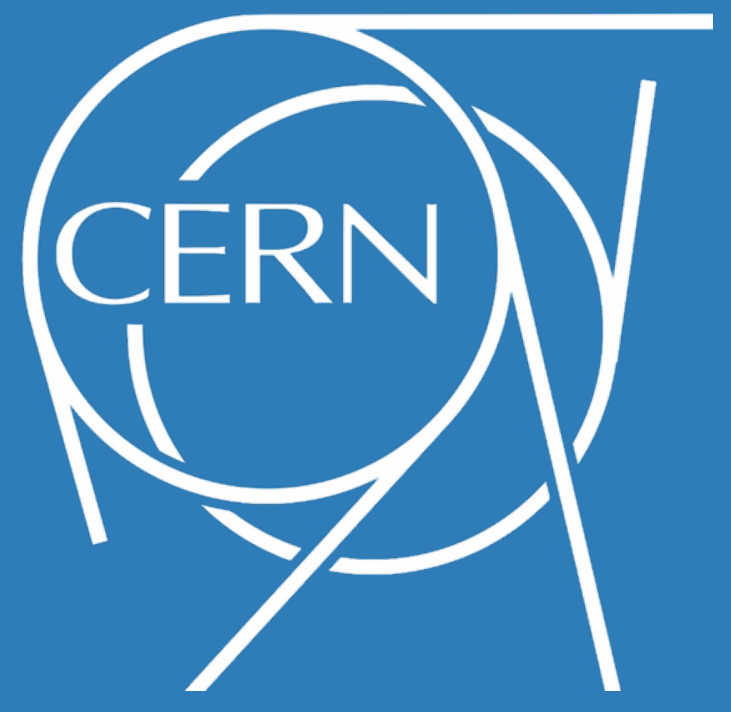




\section{Why SM?}

- "Re-discovery" of known particles

- good exercise of analysis

with increasing integrated luminosity

- Precise measurement in the standard model

- check of detector performance

- validation of the SM prediction in the energy frontier

- perturbative QCD at NLO

- background estimation for searches

- direct/indirect search for beyond SM

proton - (anti)proton cross sections

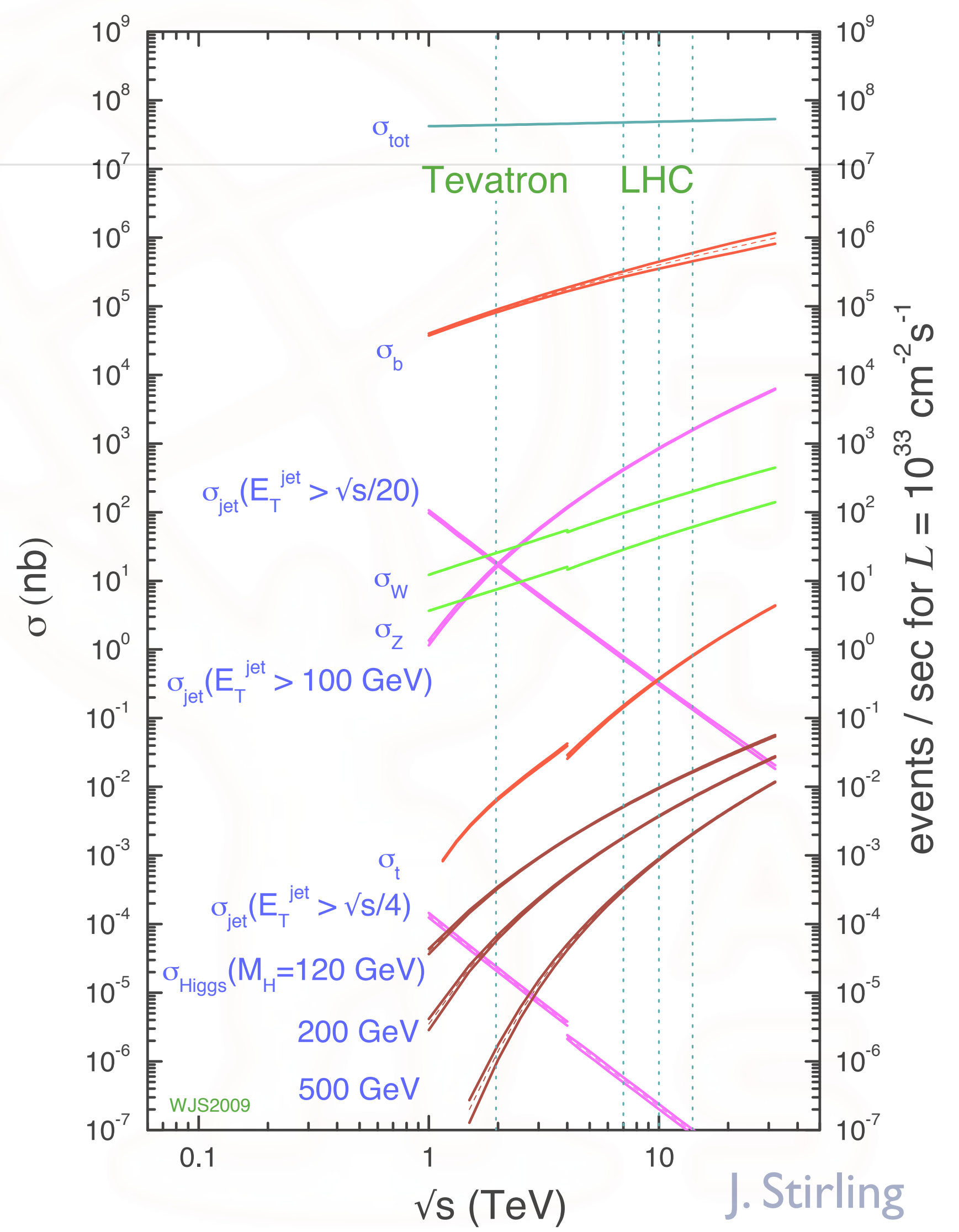




\section{Why SM?}

- "Re-discovery" of known particles

- good exercise of analysis

with increasing integrated luminosity

- Precise measurement in the standard model

- check of detector performance

- validation of the SM prediction in the energy frontier

- perturbative QCD at NLO

- background estimation for searches

- direct/indirect search for beyond SM

- Physics menus : measurements,

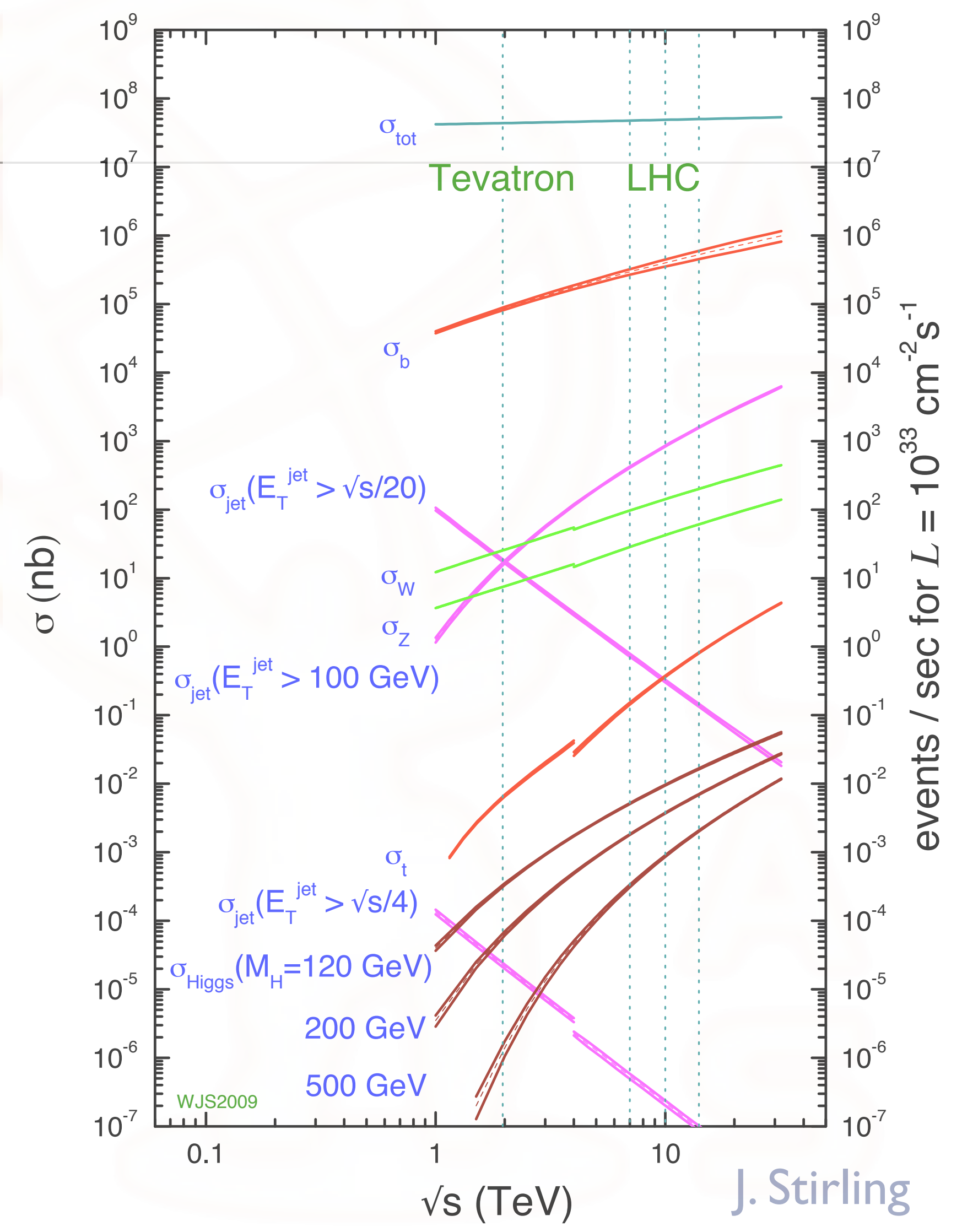




\section{Why SM?}

- "Re-discovery" of known particles

- good exercise of analysis

with increasing integrated luminosity

- Precise measurement in the standard model

- check of detector performance

- validation of the SM prediction in the energy frontier

- perturbative QCD at NLO

- background estimation for searches

- direct/indirect search for beyond SM

- Physics menus : measurements,

- Hard QCD physics $\star$

$\checkmark$ measurements of jets

- inclusive jet production

- dijet / multi-jet production

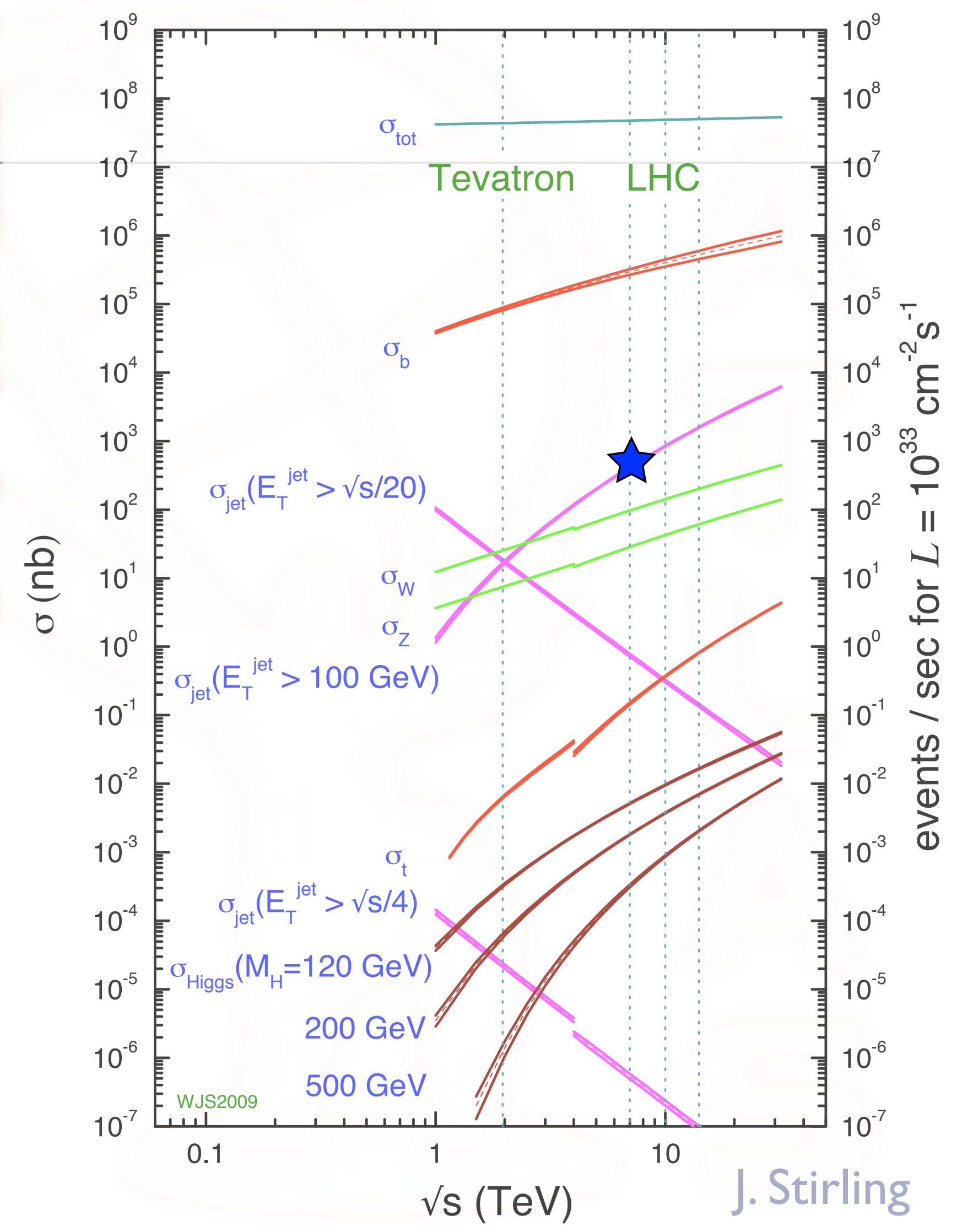




\section{Why SM?}

- "Re-discovery" of known particles

- good exercise of analysis

with increasing integrated luminosity

- Precise measurement in the standard model

- check of detector performance

- validation of the SM prediction in the energy frontier

- perturbative QCD at NLO

- background estimation for searches

- direct/indirect search for beyond SM

- Physics menus : measurements,

- Hard QCD physics $\star$

$\checkmark$ measurements of jets

- inclusive jet production

- dijet / multi-jet production

- Electroweak $\star$

- inclusive $W / Z$ cross section measurements

- $W / Z+$ jets

- diboson production

proton - (anti)proton cross sections

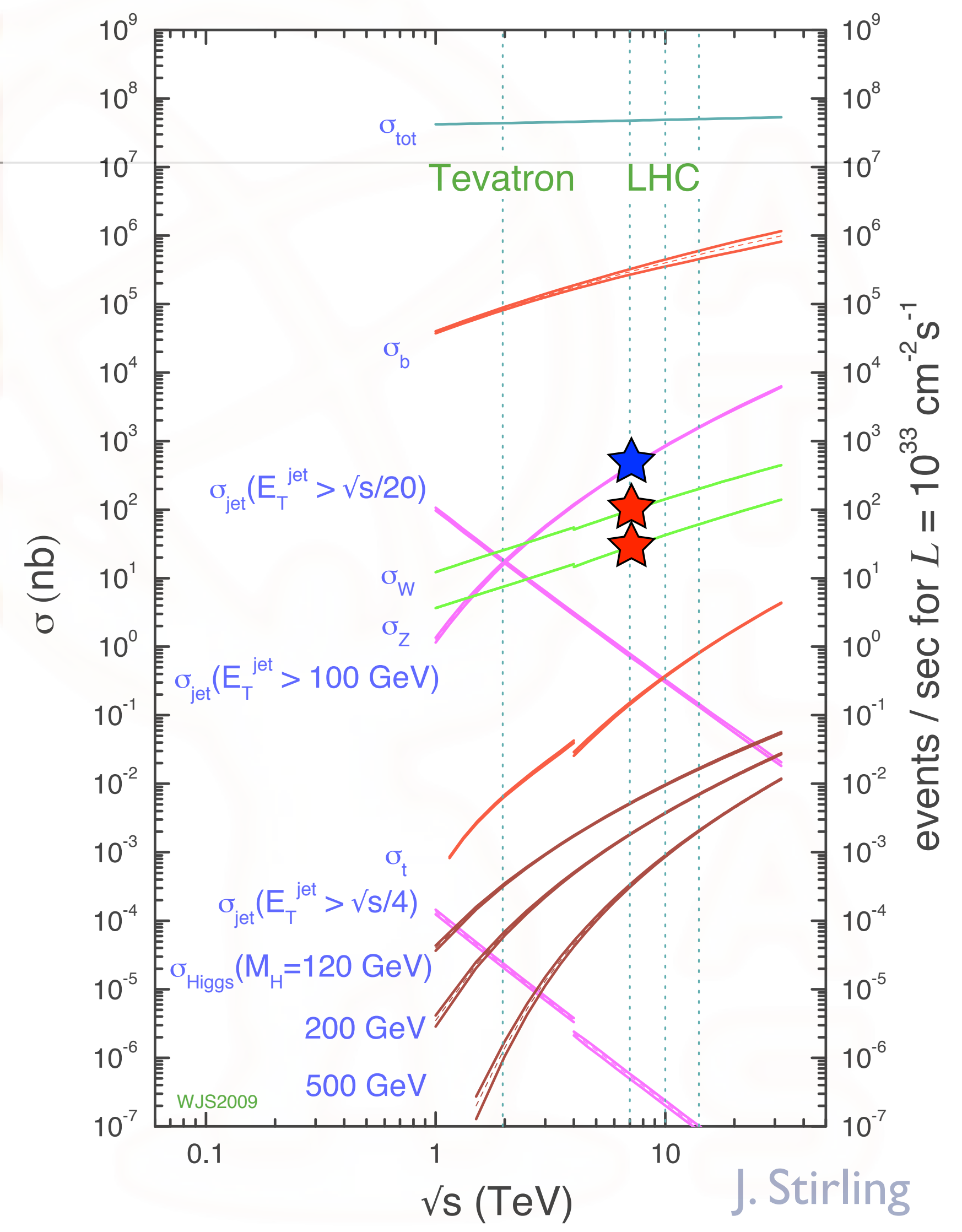




\section{Why SM?}

proton - (anti)proton cross sections

- "Re-discovery" of known particles

- good exercise of analysis

with increasing integrated luminosity

- Precise measurement in the standard model

- check of detector performance

- validation of the SM prediction in the energy frontier

- perturbative QCD at NLO

- background estimation for searches

- direct/indirect search for beyond SM

- Physics menus : measurements,

- Hard QCD physics $\star$

$\checkmark$ measurements of jets

- inclusive jet production

- dijet / multi-jet production

- Electroweak $\star$

- inclusive $W / Z$ cross section measurements

- $W / Z+$ jets

- diboson production

- Top

- pair production cross section, mass measurement

- single top quark production

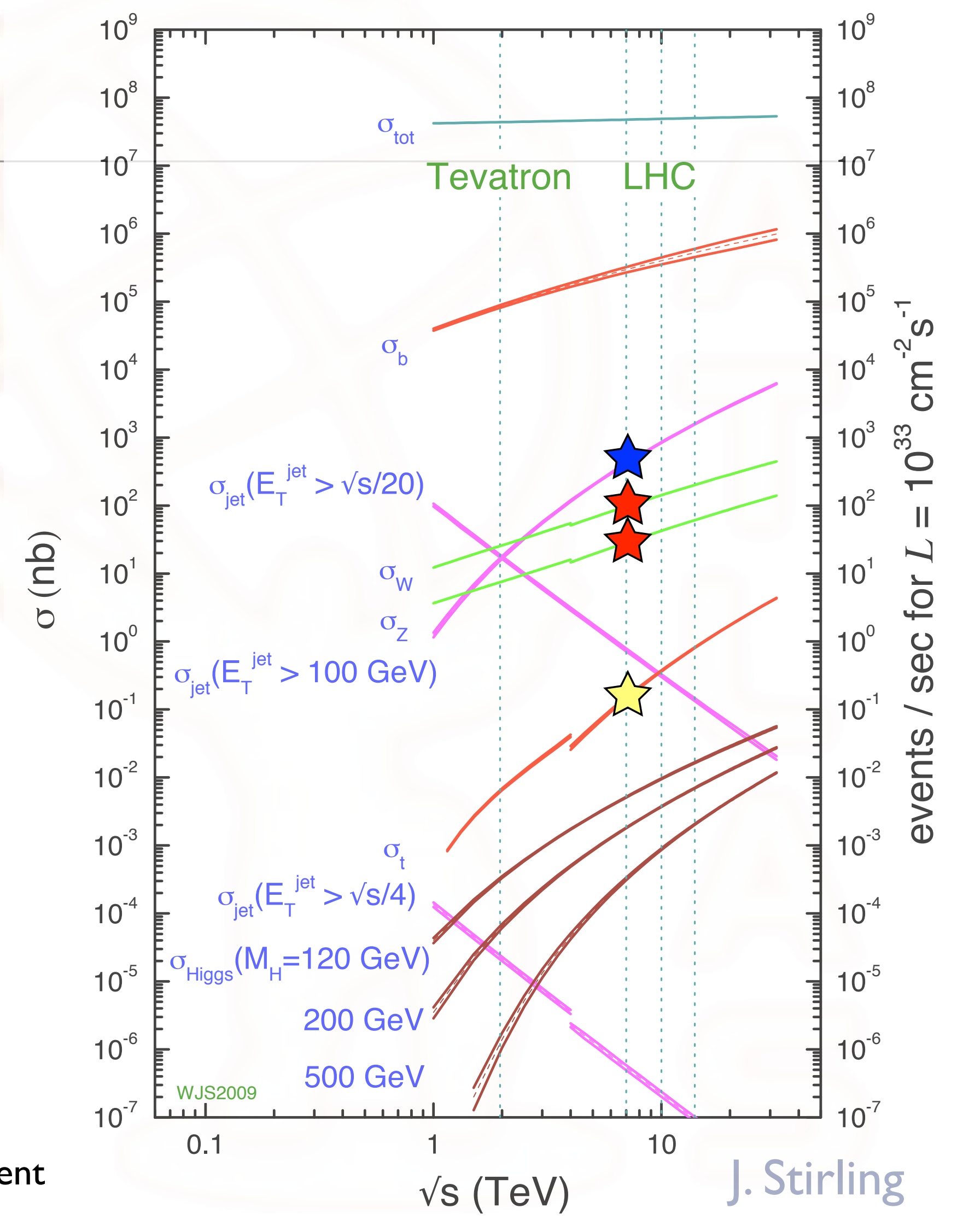




\section{LHC and ATLAS}


- Large Hadron Collider

- The world's largest and highest energy proton-proton $(\mathrm{Pb}-\mathrm{Pb})$ collider at CERN ( Geneva, Switzerland )

- circumference: $27 \mathrm{~km}$

- max. energy: 7+7 TeV (p-p)

- 4+2 experiments

- ATLAS, CMS

- general purpose

- LHC-B

- B physics

- ALICE

- Heavy lon collision

- LHCf,TOTEM

- forward particles

- total cross section

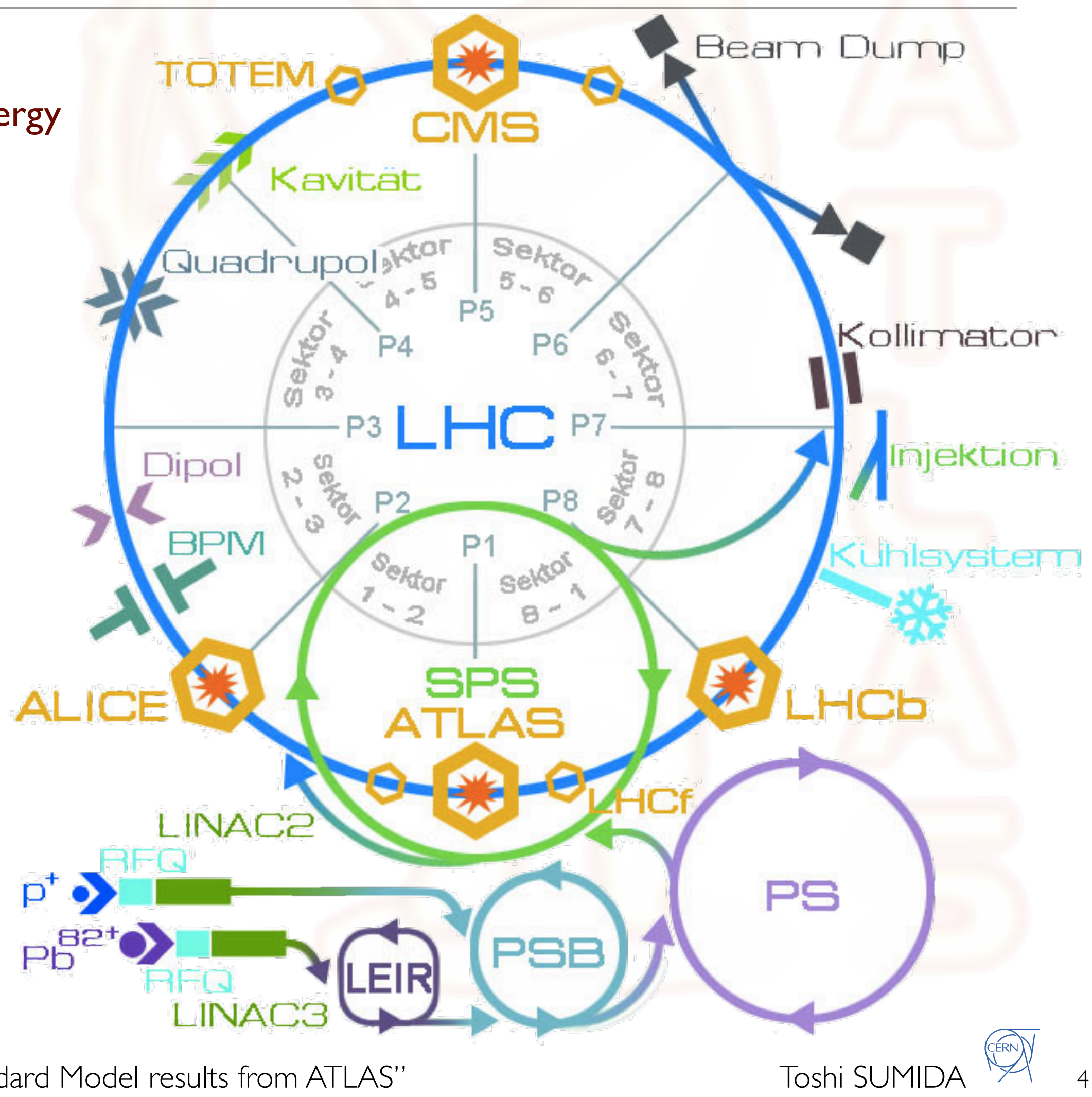




\section{LHC status}

- 2010/20II run

- $\sqrt{ } \mathrm{s}: 3.5+3.5=7 \mathrm{TeV}$

- peak luminosity

- $2.1 \times 10^{32} / 1.3 \times 10^{33} \mathrm{~cm}^{-2} \mathrm{~s}^{-1}$

- integrated luminosity

- 45 / 1 100 $\mathrm{pb}^{-1}$

\section{$\checkmark$ reached $\mathrm{Ifb}^{-1} ! !$}

\begin{tabular}{|l|c|c|c|}
\hline \multicolumn{1}{|c|}{ Parameter } & 2010 & 2011 & Nominal \\
\hline Beam energy & $3.5 \mathrm{TeV}$ & $3.5 \mathrm{TeV}$ & $7 \mathrm{TeV}$ \\
\hline Beam squeeze & $3.5 \mathrm{~m}$ & $<1.5 \mathrm{~m}$ & $0.55 \mathrm{~m}$ \\
\hline Transverse emittance & $2-3 \mu \mathrm{m} \mathrm{rad}$ & $2.5 \mu \mathrm{m} \mathrm{rad}$ & $3.75 \mu \mathrm{m} \mathrm{rad}$ \\
\hline Protons per bunch & up to $1.2 \times 10^{11}$ & $1.2 \times 10^{11}$ & $1.15 \times 10^{11}$ \\
\hline Bunch separation & $150 \mathrm{~ns}$ & $50 \mathrm{~ns}$ & $25 \mathrm{~ns}$ \\
\hline Number of bunches & 368 & $>1000$ & 2808 \\
\hline max peak luminosity $\left(\mathrm{cm}^{-2} \mathrm{~s}^{-1}\right)$ & $2.1 \times 10^{32}$ & $1.3 \times 10^{33}$ & $>10^{34}$ \\
\hline
\end{tabular}
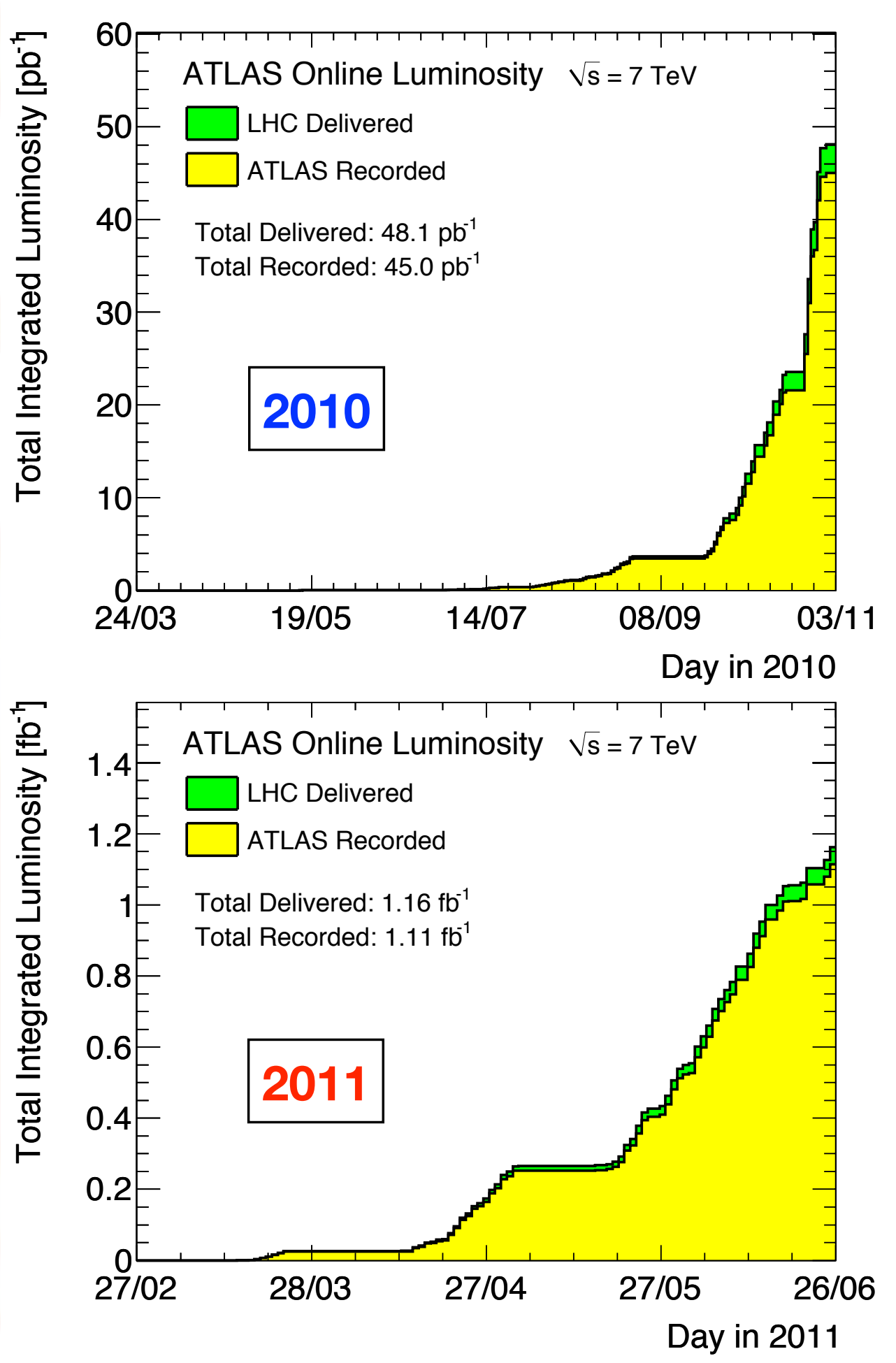

Toshi SUMIDA 


\section{ATLAS}

- A Toroidal LHC ApparatuS

- multipurpose detector designed to cover all the expected physics channels at the $\mathrm{TeV}$ energy scale

- Collaboration

- 3000 scientists

- more than 1000 $\mathrm{PhD}$ students

- 174 institutions

- 38 countries

- The ATLAS detector

- weight: 7000 tons

- height: $25 \mathrm{~m}$

- length: $45 \mathrm{~m}$

- $\sim 10^{8}$ channels

- $(90 \%$ in the tracker)

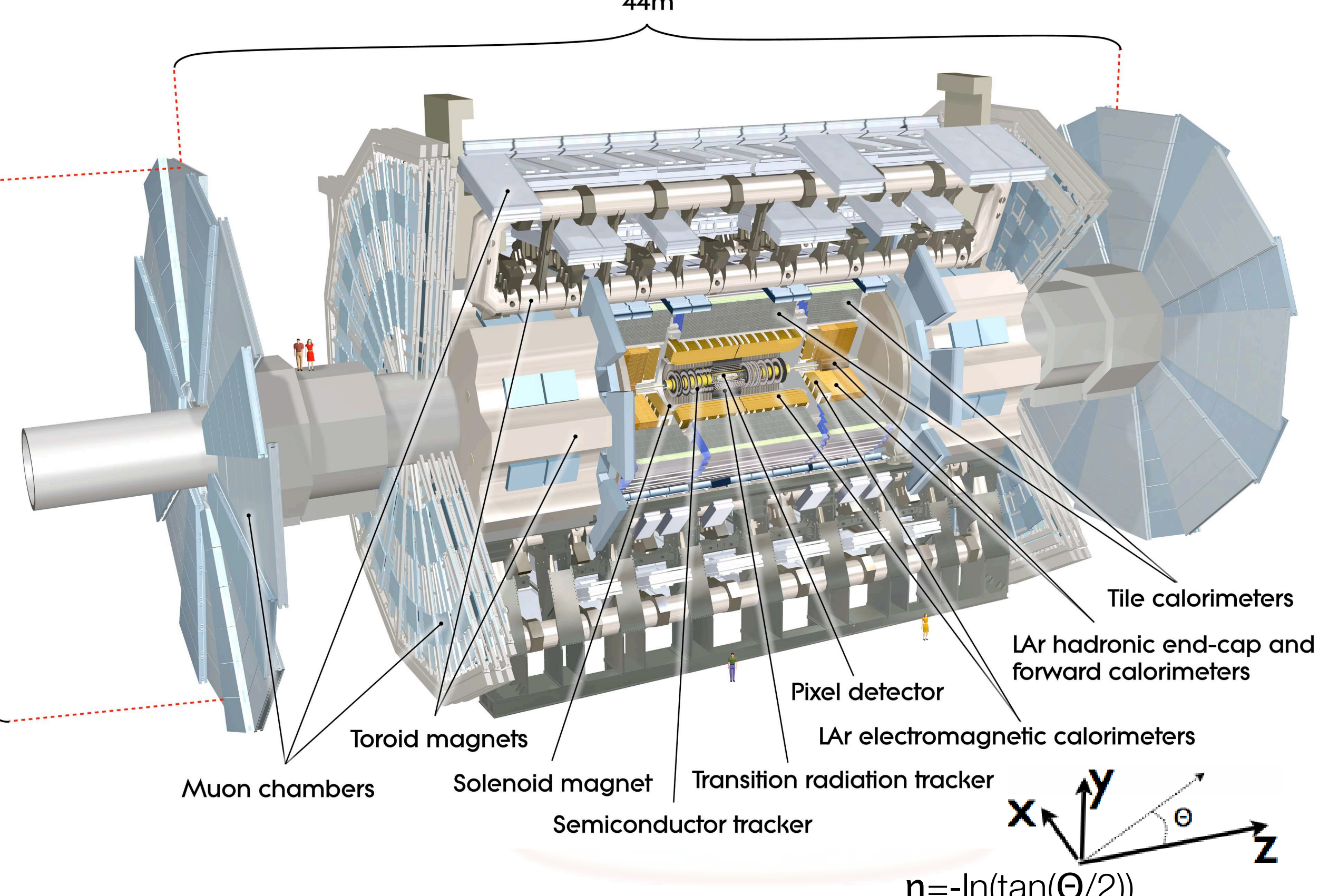

$$
\eta=-\ln (\tan (\Theta / 2))
$$




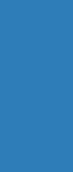

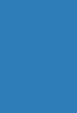

\section{Jet production}

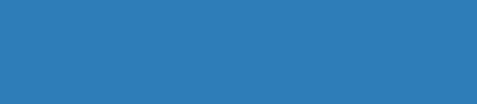

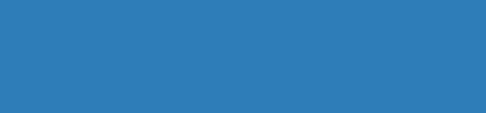

(2) 


\section{What are Jets?}

- Collimated bunches of stable hadrons

- originating from partons (quarks \& gluons) after fragmentation/hadronization

- Difficulty in the jet measurement

- need to understand every stage

- Prediction by theory

- parton distribution

$\checkmark$ quark/gluon

- hadronization

- Jet Finding

- approximate attempts to reverse-engineer the quantum mechanical processes of hadronization

- Calorimeter response

- in the EM scale

- to hadrons

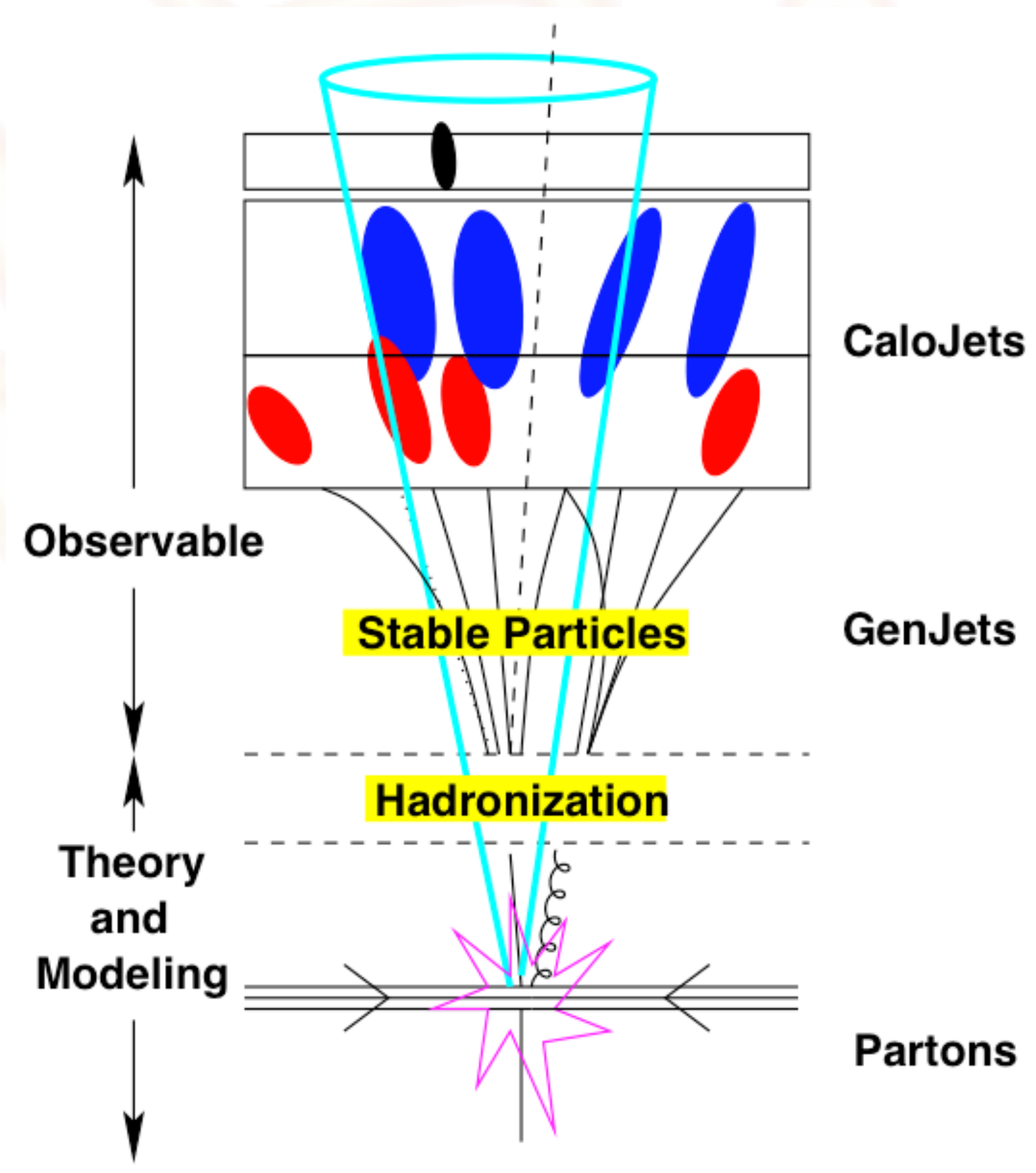




\section{Jet reconstruction}

- Constituent : input for reconstruction

- TopoCluster : 3D, default

- 4-2-0 noise suppression using pedestal $\sigma$

- TopoTower :2D

- w/ noise suppression

- (Tower)

- w/o noise suppression

- Anti-kt algorithm

- infrared-safe and Collinear-safe
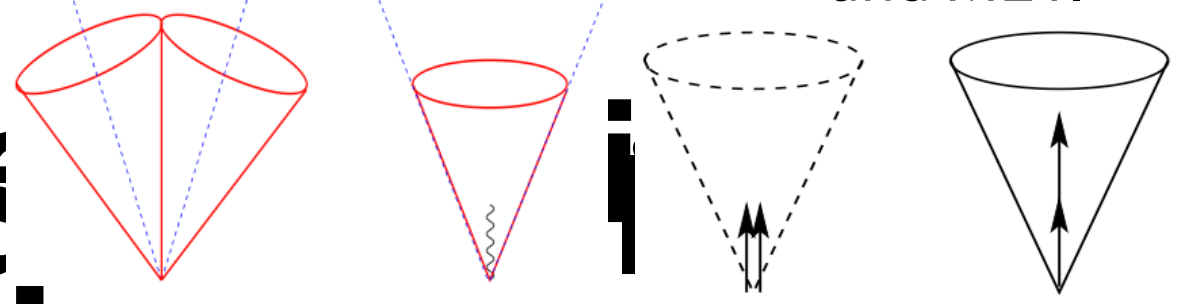

- based on the distance measures:

- $d_{i j}=\min \left(k_{T i}^{2 p}, k_{T j}^{2 p}\right) \frac{\Delta_{i j}}{R}$

$p=-1:$ anti- $k_{T}$

$\checkmark$ between two constituents

$\Delta_{i j}^{2}=\left(y_{i}-y_{j}\right)^{2}+\left(\phi_{i}-\phi_{j}\right)^{2}$

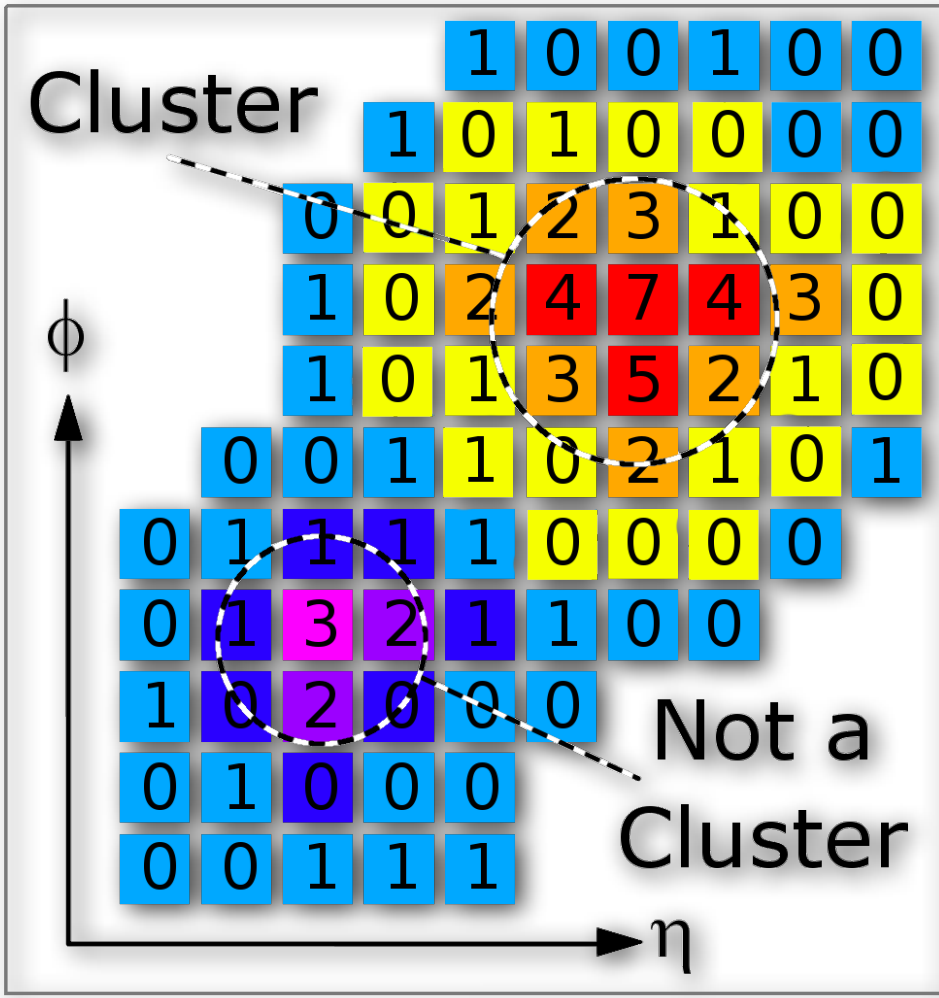

$d_{i B}=k_{\mathrm{T} i}^{2 p}$

$\checkmark$ between any constituents and the beam

- ATLAS: Anti-kT TopoCluster jet with $\mathrm{R}=0.4,0.6$

- larger size jet study ongoing

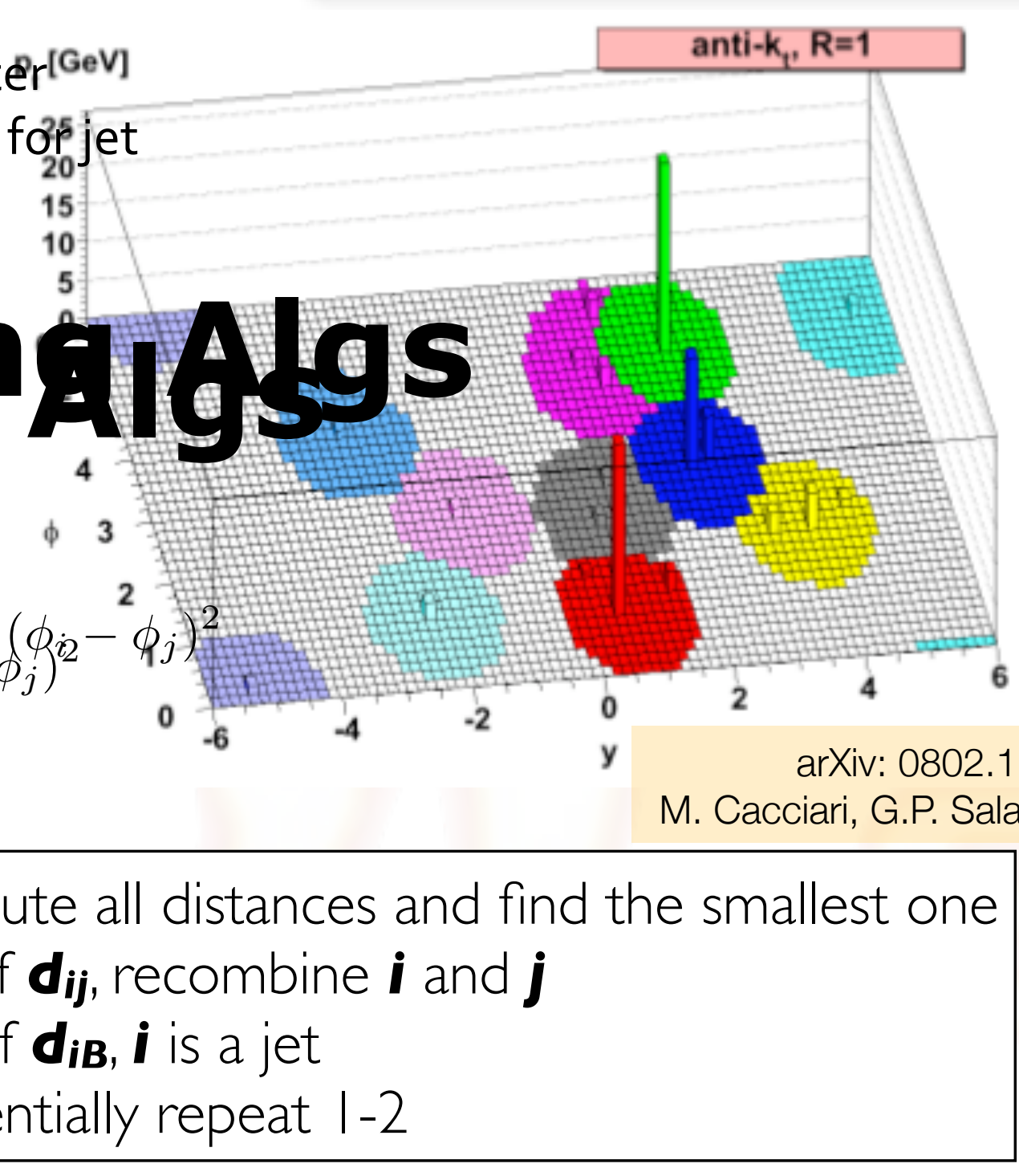




\section{Event display}

- a dijet event in 2010 with the highest invariant mass

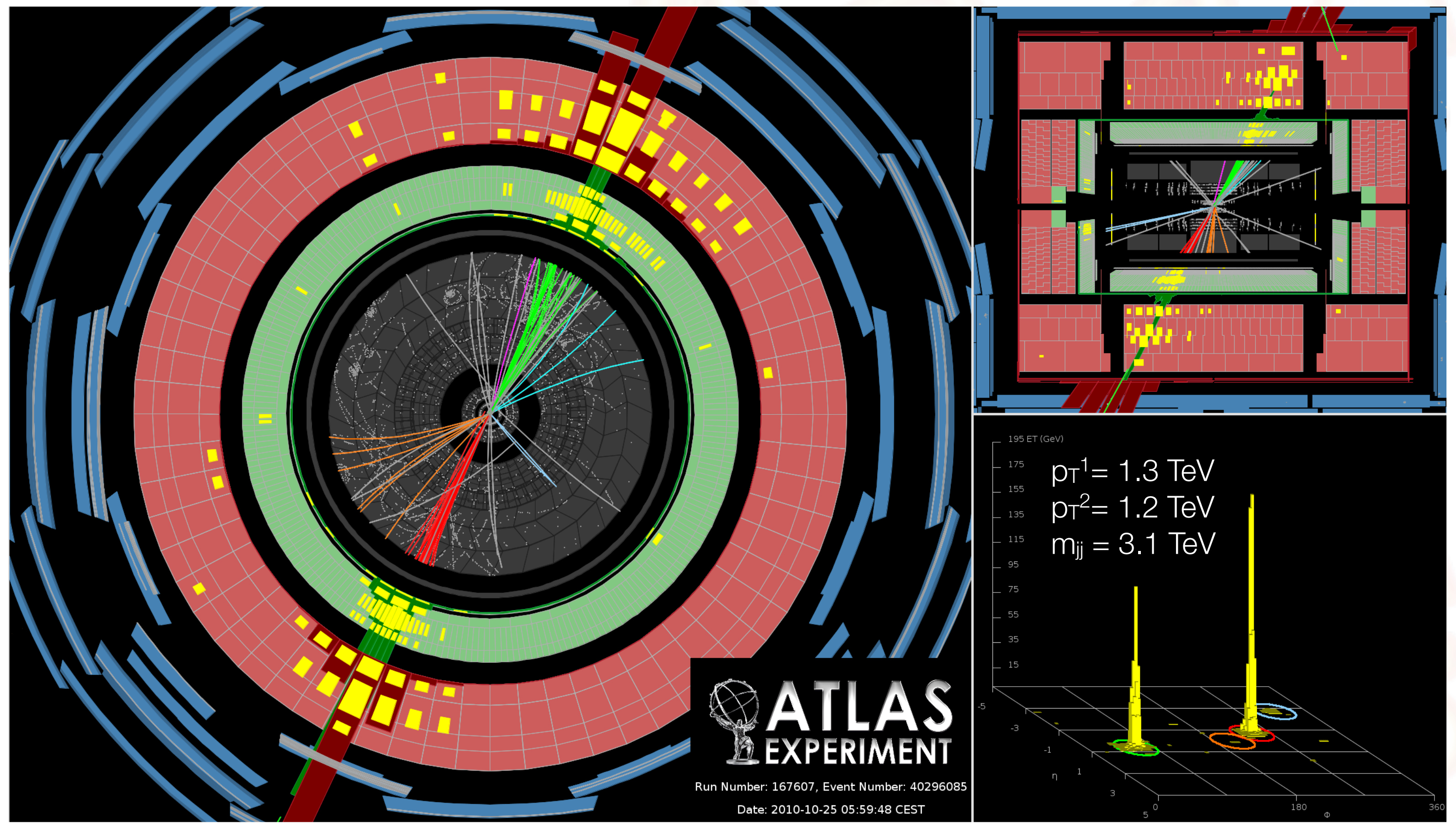




\section{Jet calibration}

- Calibration constant :"jet energy scale" (JES)

- derived from Monte Carlo (MC) truth information

- (

\section{- EM+JES}

- default calibration

- well understood uncertainty

- JES uncertainty (for 2010 data)

- based on single hadron response method

- MC model dependence

- extension to forward region

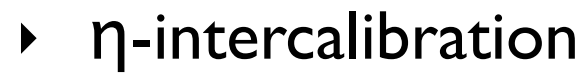

- Pile-up

- energy from other primary vertices
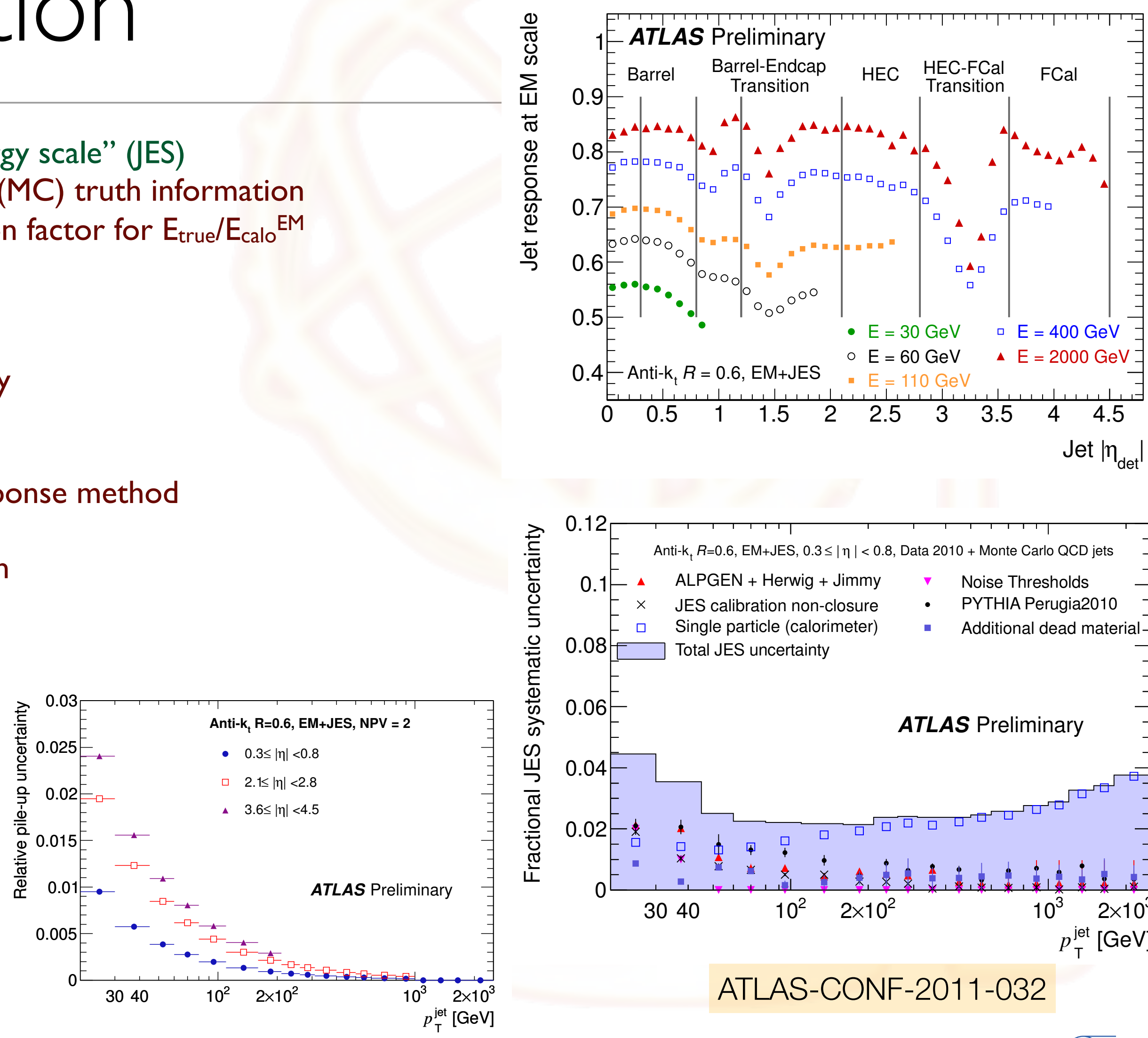

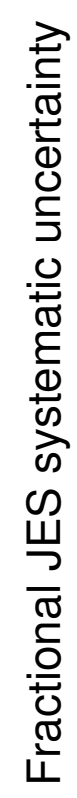

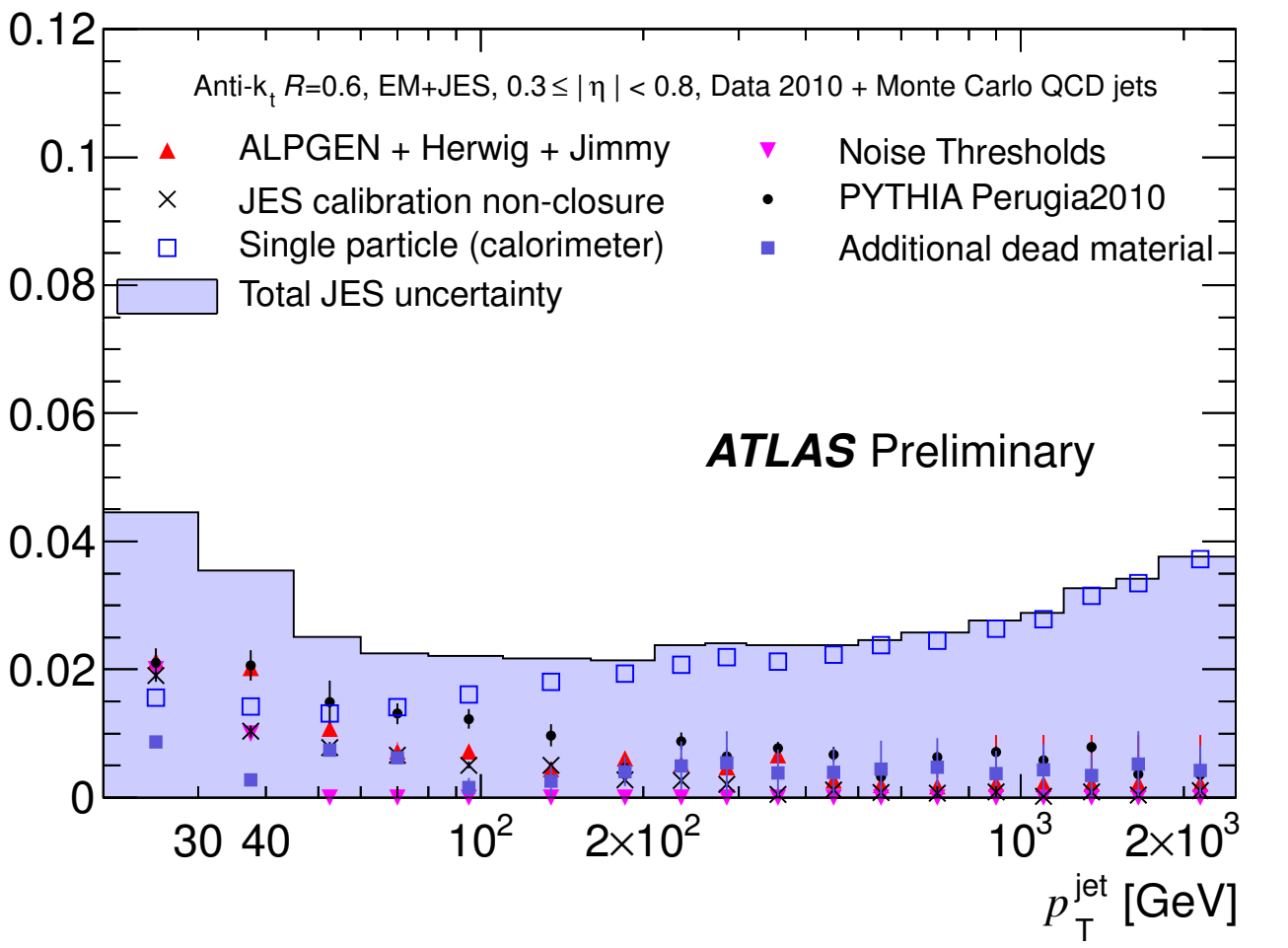

ATLAS-CONF-2011-032 


\section{Jet production measurements}

- Results in 2010 data (36-37 $\left.\mathrm{pb}^{-1}\right)$

- Inclusive jet cross section

- Dijet production

- B-jet production

- Dijet azimuthal decorrelation

- Dijet production with a jet veto

- Multi-jet production (2.43 $\left.\mathrm{pb}^{-1}\right)$

- Jet mass and substructure 


\section{Jet production measurements}

- Results in 2010 data $\left(36-37 \mathrm{pb}^{-1}\right)$

- Inclusive jet cross section

- Dijet production

- Dijet azimuthal decorrelation

- Multi-jet production $\left(2.43 \mathrm{pb}^{-1}\right)$ 


\section{Jet production measurements}

- Results in 2010 data $\left(36-37 \mathrm{pb}^{-1}\right)$

- Inclusive jet cross section

- Dijet production

- Dijet azimuthal decorrelation

- Multi-jet production $\left(2.43 \mathrm{pb}^{-1}\right)$

- Skipped results and details

- https://atlas.web.cern.ch/Atlas/GROUPS/PHYSICS/CONFNOTES/ 


\section{Inclusive jet cross section}

- Event selection

- at least I primary vertex

- single jet trigger

- jet quality selection criteria

- to remove non-collision jets

- Theoretical predictions

- NLO calculations (NLOJET++ 4.I.2 + Applgrid) with non-perturbative corrections (Pythia, AMBTI)

- NLO parton shower MC using POWHEG

- uncertainties in predictions

- PDF

- factorisation (hadronization)

- renormalisation scale

- $\alpha s$

- Unfolding

- to recover true distribution of jet PT calculating migration of jets

- bin-by-bin using $M C$

- Results in 2010 data $\left(37 \mathrm{pb}^{-1}\right)$

- e.g. ) anti-kT $R=0.6$

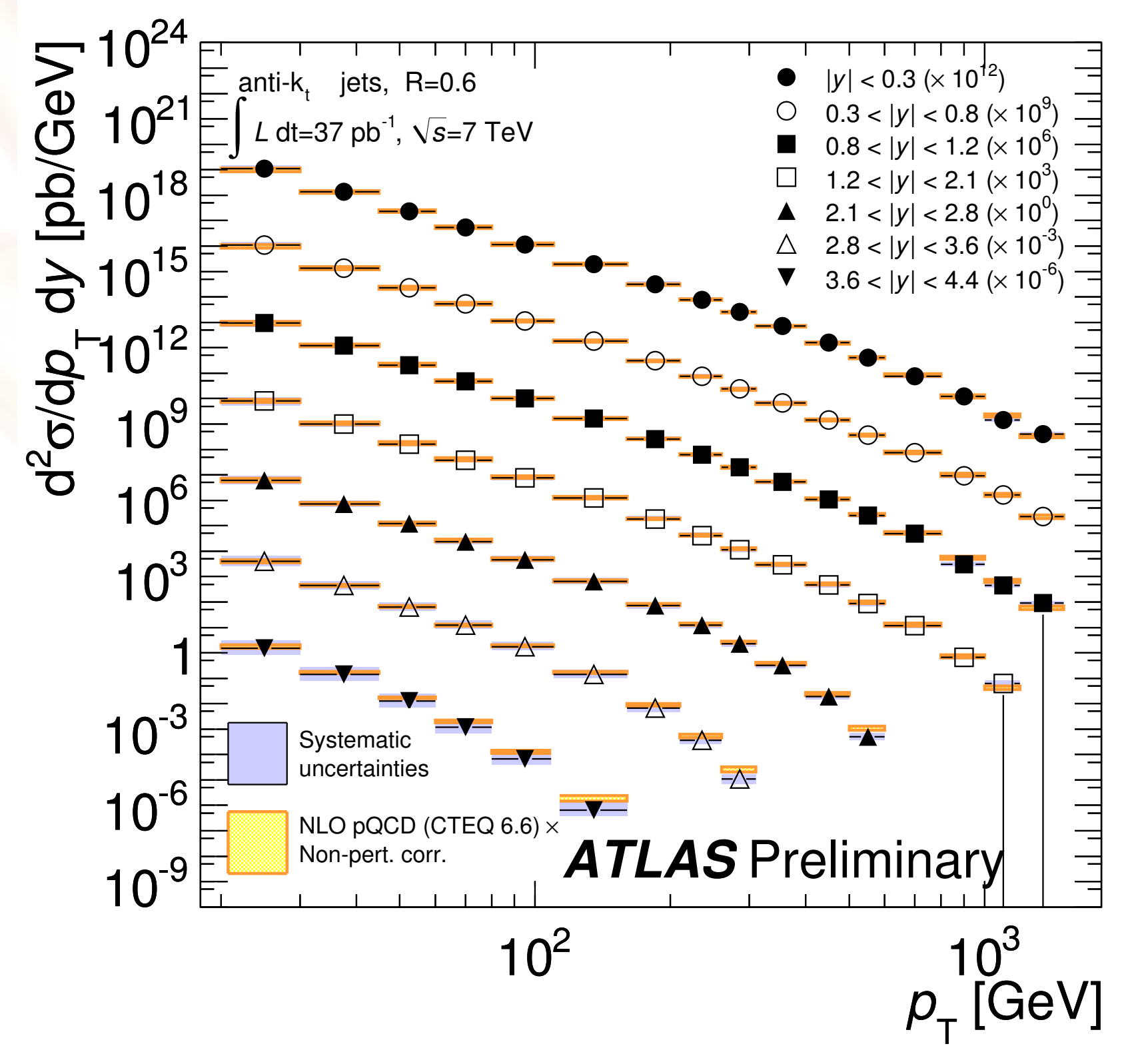

- $\mathrm{PT}>20 \mathrm{GeV},|y|<4.4$

- good agreement over 9 orders of magnitude!! 


\section{Dijet cross section}

- Double-differential dijet cross section

- $\mathrm{PT}^{1}>30 \mathrm{GeV}, \mathrm{PT}^{2}>20 \mathrm{GeV}$

- $|y|<2.8$

- Systematic uncertainty

- $20 \%$
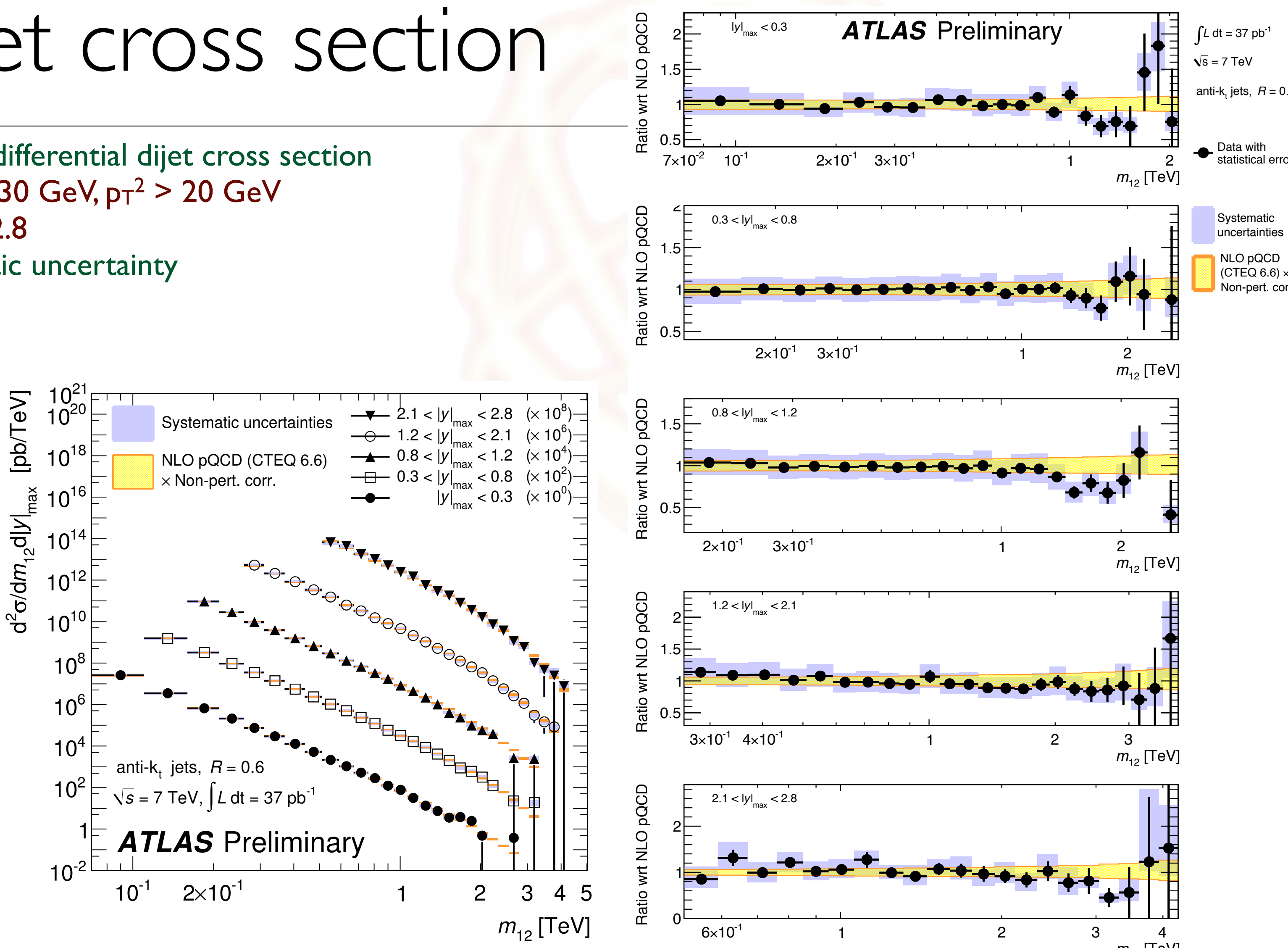

Systematic uncertainties

NLO pQCD (CTEQ 6.6) $\times$
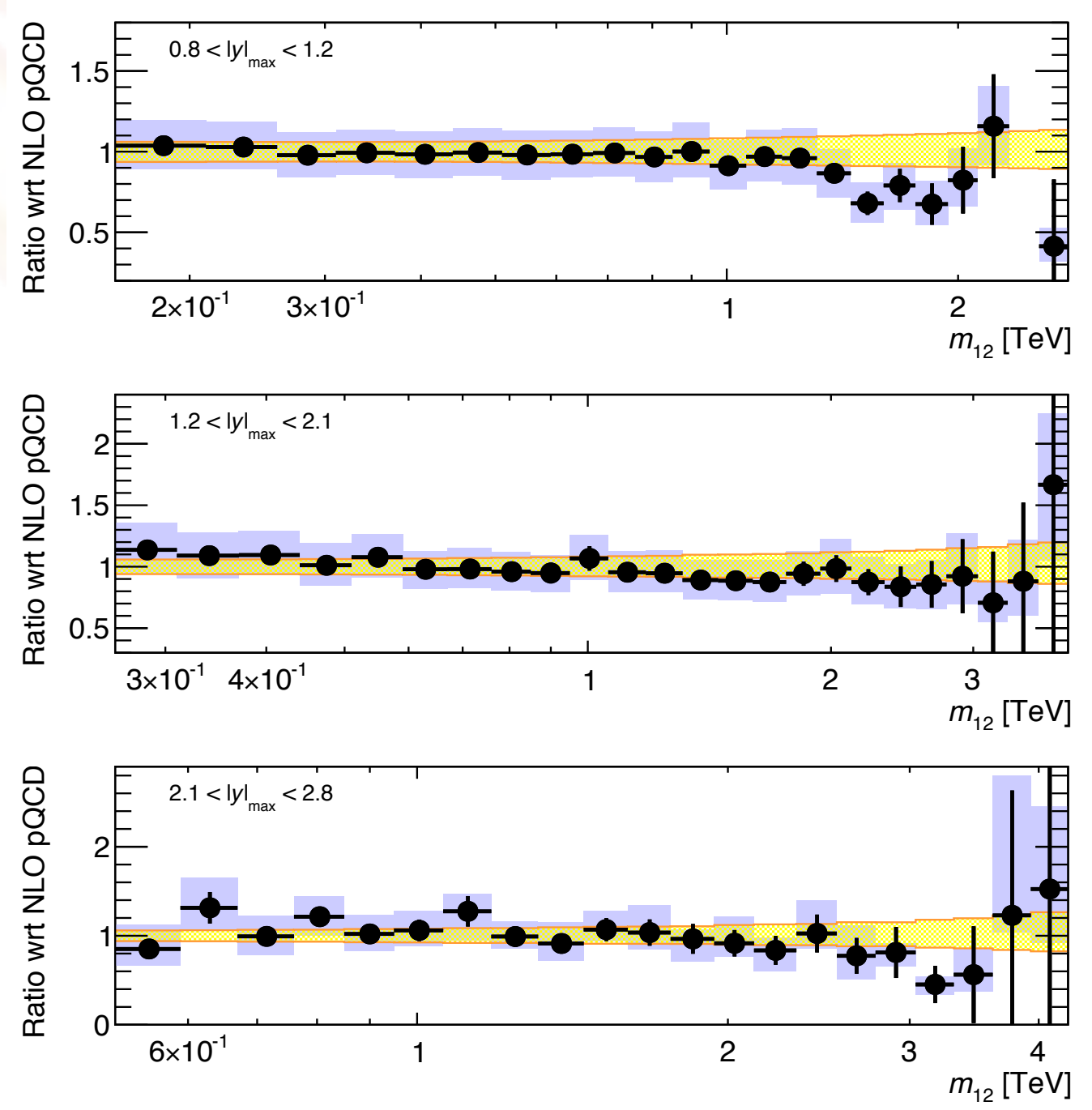


\section{Dijet azimuthal decorrelation}

- $\Delta \varphi$ of leading jets

- depends on number of partons

\section{$\checkmark$ sensitive test of QCD predictions}

- Uncertainties

- JES: $2-17 \%$

- unfolding: $1-19 \%$

- jet energy and position resolution: $0.5-5 \%$

- Results

- relatively good agreement with similar size of uncertainties in data and theory
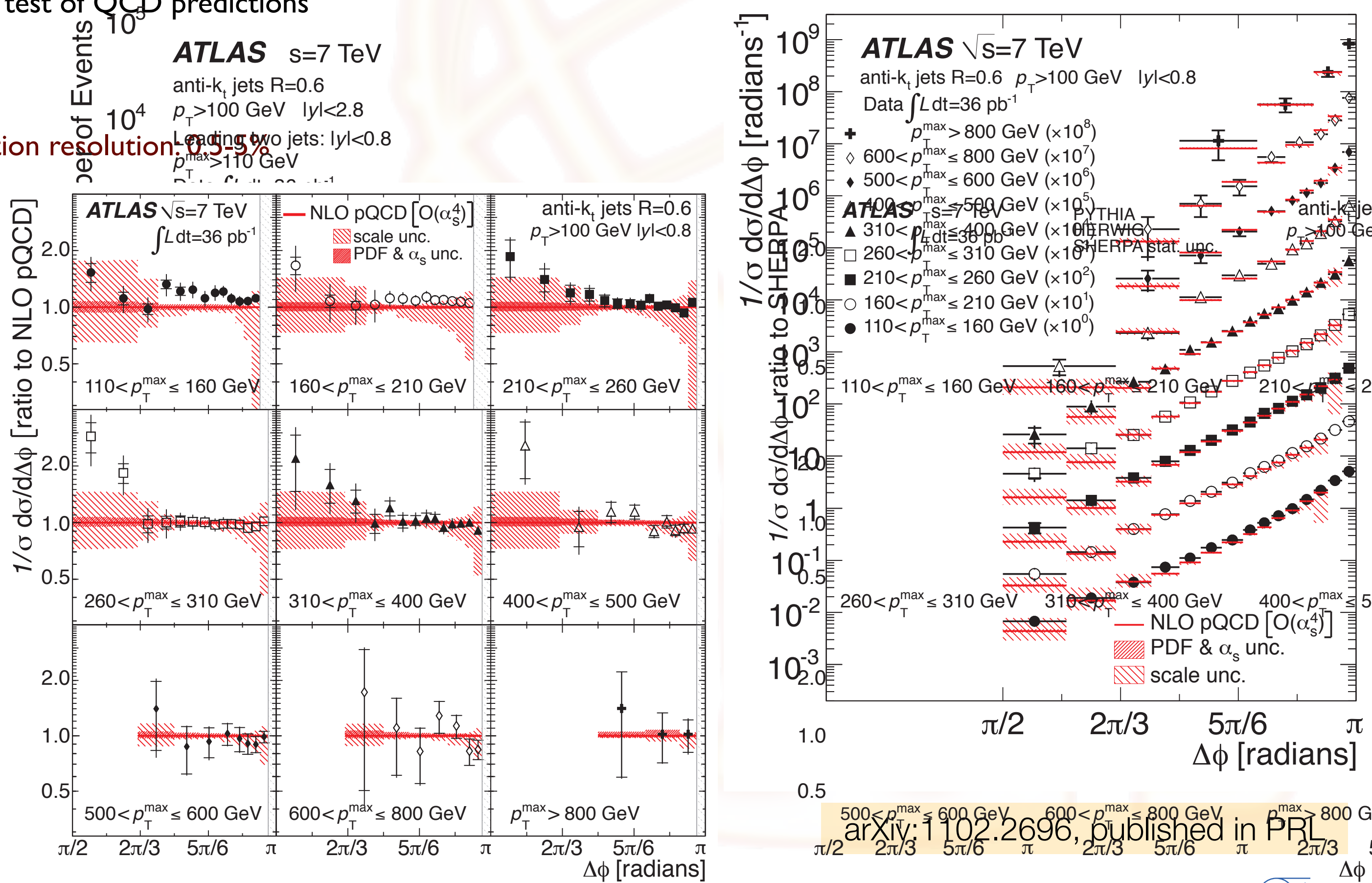

arXiv:1102.2696, published in PRL 


\section{Multi-jet production}

- Motivation

- multi-jet probe higher-order perturbative QCD

- cross section ratios are calculated at NLO and can be used to extract $\alpha$ s

- understand as a background source to searches

- Inclusive jet multiplicity

- JES is the dominant systematic uncertainty

- Ratio of the $n$-jet cross section to the (n-I) jet cross section

- systematics reduced

- Alpgen describes better than Pythia

- Differential cross section vs. $H_{T}$

- scalar sum of the PT of the selected jets

- e.g. $(\geq 3$ jets $) /(\geq 2$ jets $)$

- Pythia

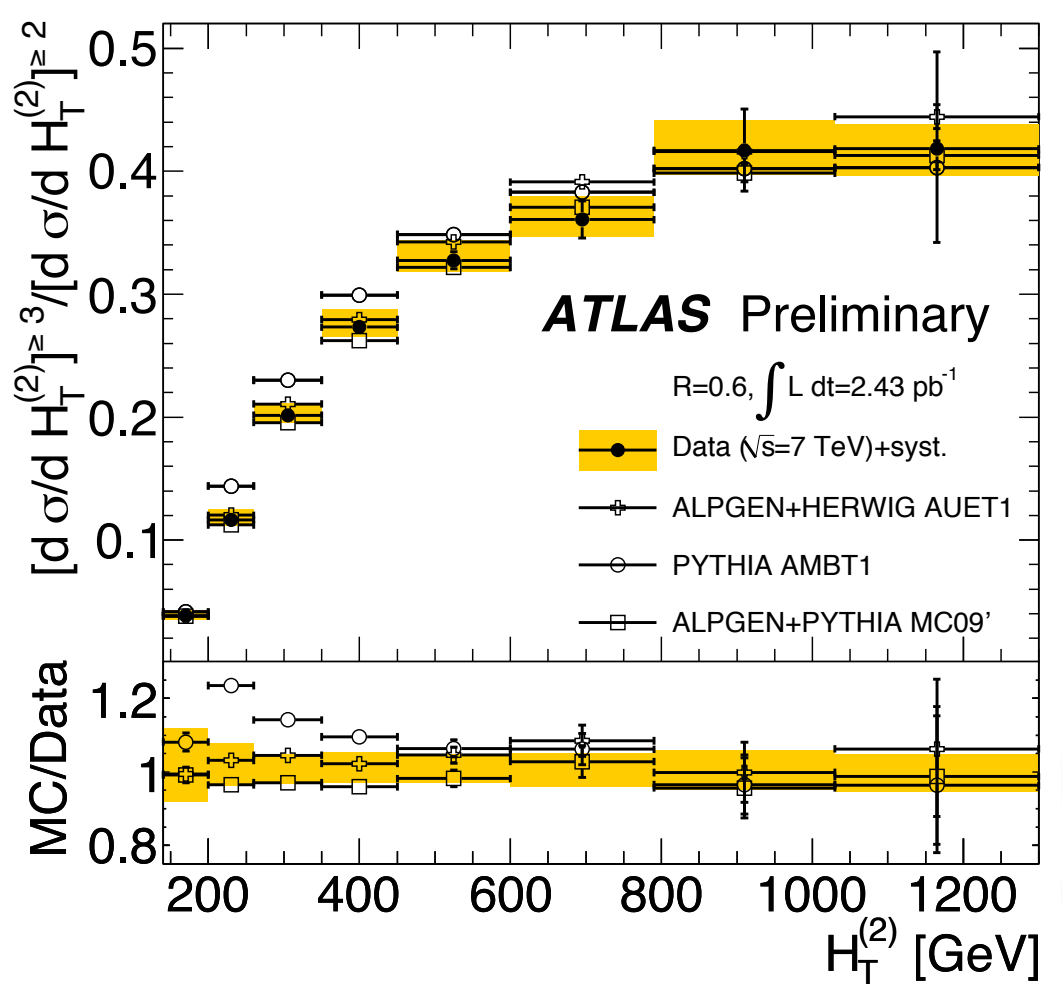

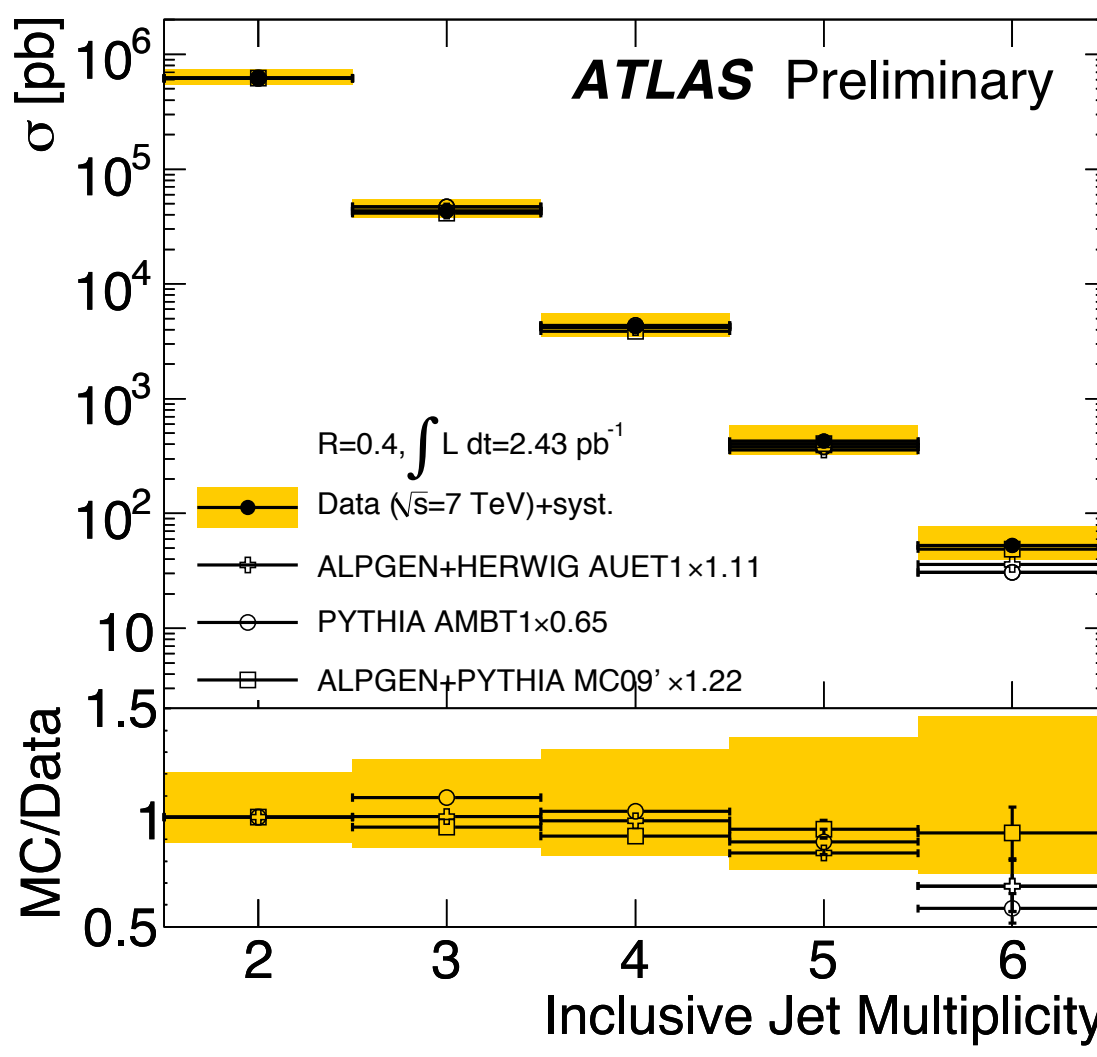

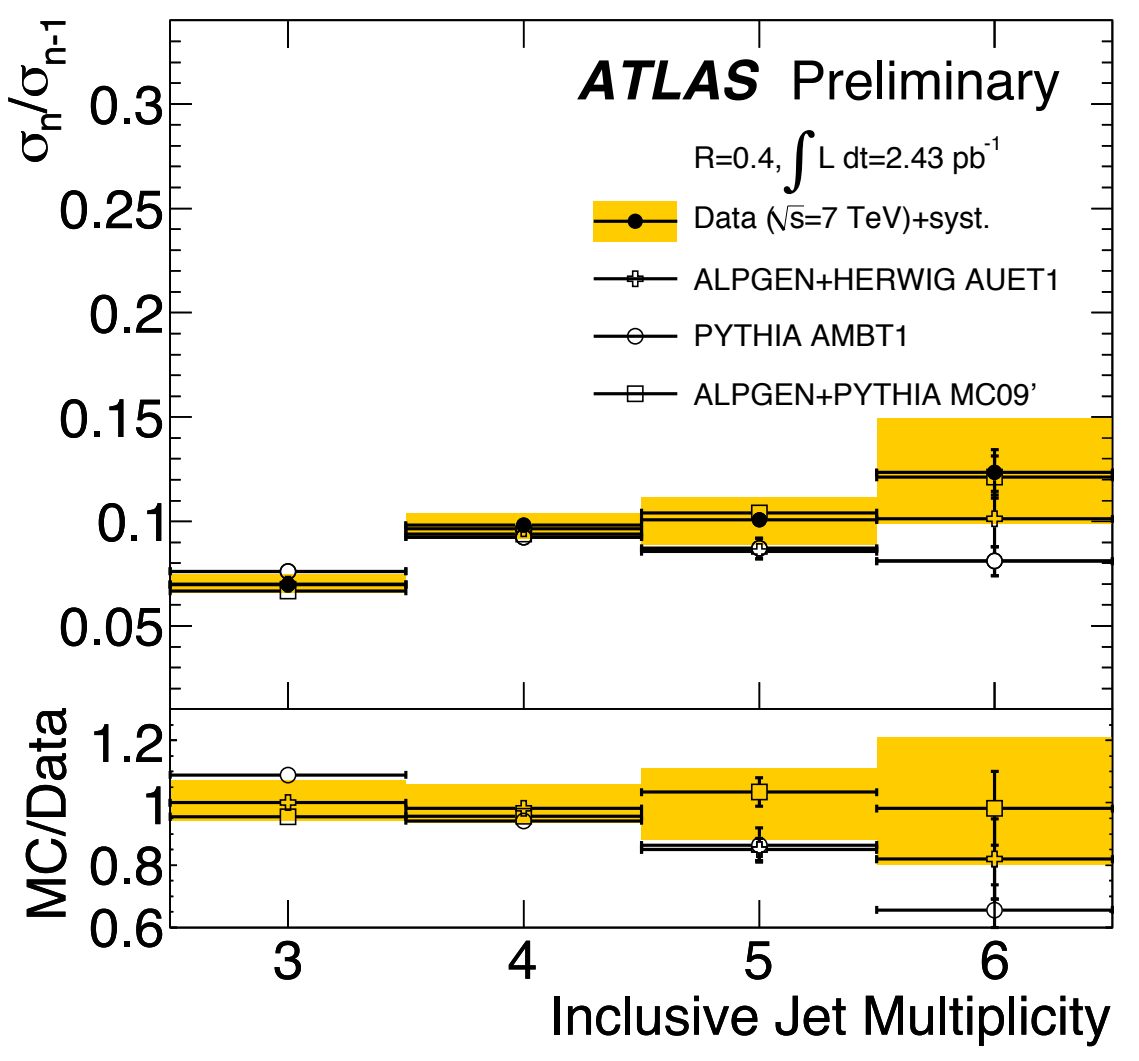

ATLAS-CONF-2011-043 
Electroweak Bosons

\section{Electroweak Bosons (n)}

(1) 


\section{W/Z production measurements}

- W/Z production cross section

- cross section ratios

- Properties

- W charge asymmetry

- Z $\rightarrow$ TT measurement

- Production associated with jets

- W + jets

- Z + jets

- Diboson production

- Wy / Z Y production

- WW production

- WZ production 


\section{W/Z production measurements}

- W/Z production cross section

- cross section ratios

- Production associated with jets

- W + jets

- Diboson production

- Wy / Z y production

- WW production

- WZ production 


\section{W/Z production cross section}

- Inclusive cross section measurement

- Event selection

- $W \rightarrow \mid V$

- $\mathrm{PT}^{\mathrm{I}}>20 \mathrm{GeV}$

- $\mathrm{PT}^{\mathrm{V}}>25 \mathrm{GeV}$

- $\mathrm{m}_{\mathrm{T}}>40 \mathrm{GeV}$

- $\mathrm{Z} \rightarrow \|$

- $\mathrm{PT}^{\top}>20 \mathrm{GeV}$

- $66<\mathrm{m} \|_{\|}<116 \mathrm{GeV}$

- extended to one forward electron

$$
\text { - } 2.5<|\eta|<4.9
$$

- Background estimation

- QCD background

- data-driven methods

- EW background

- based on MC predictions

- Results

\begin{tabular}{l|c} 
& $\sigma_{W^{( \pm)}}^{\text {tot }} \cdot \mathrm{BR}(W \rightarrow \ell v) \quad[\mathrm{nb}]$ \\
\hline$W^{+}$ & $6.257 \pm 0.017(\mathrm{sta}) \pm 0.152(\mathrm{sys}) \pm 0.213(\mathrm{lum}) \pm 0.188(\mathrm{acc})$ \\
$W^{-}$ & $4.149 \pm 0.014(\mathrm{sta}) \pm 0.102(\mathrm{sys}) \pm 0.141(\mathrm{lum}) \pm 0.124(\mathrm{acc})$ \\
$W$ & $10.391 \pm 0.022(\mathrm{sta}) \pm 0.238(\mathrm{sys}) \pm 0.353(\mathrm{lum}) \pm 0.312(\mathrm{acc})$ \\
\hline \hline & $\sigma_{Z / \gamma^{*}}^{\text {tot }} \cdot \mathrm{BR}\left(Z / \gamma^{*} \rightarrow \ell \ell\right)[\mathrm{nb}], 66<m_{e e}<116 \mathrm{GeV}$ \\
\hline$Z / \gamma^{*}$ & $0.945 \pm 0.006(\mathrm{sta}) \pm 0.011(\mathrm{sys}) \pm 0.032(\mathrm{lum}) \pm 0.038(\mathrm{acc})$
\end{tabular}
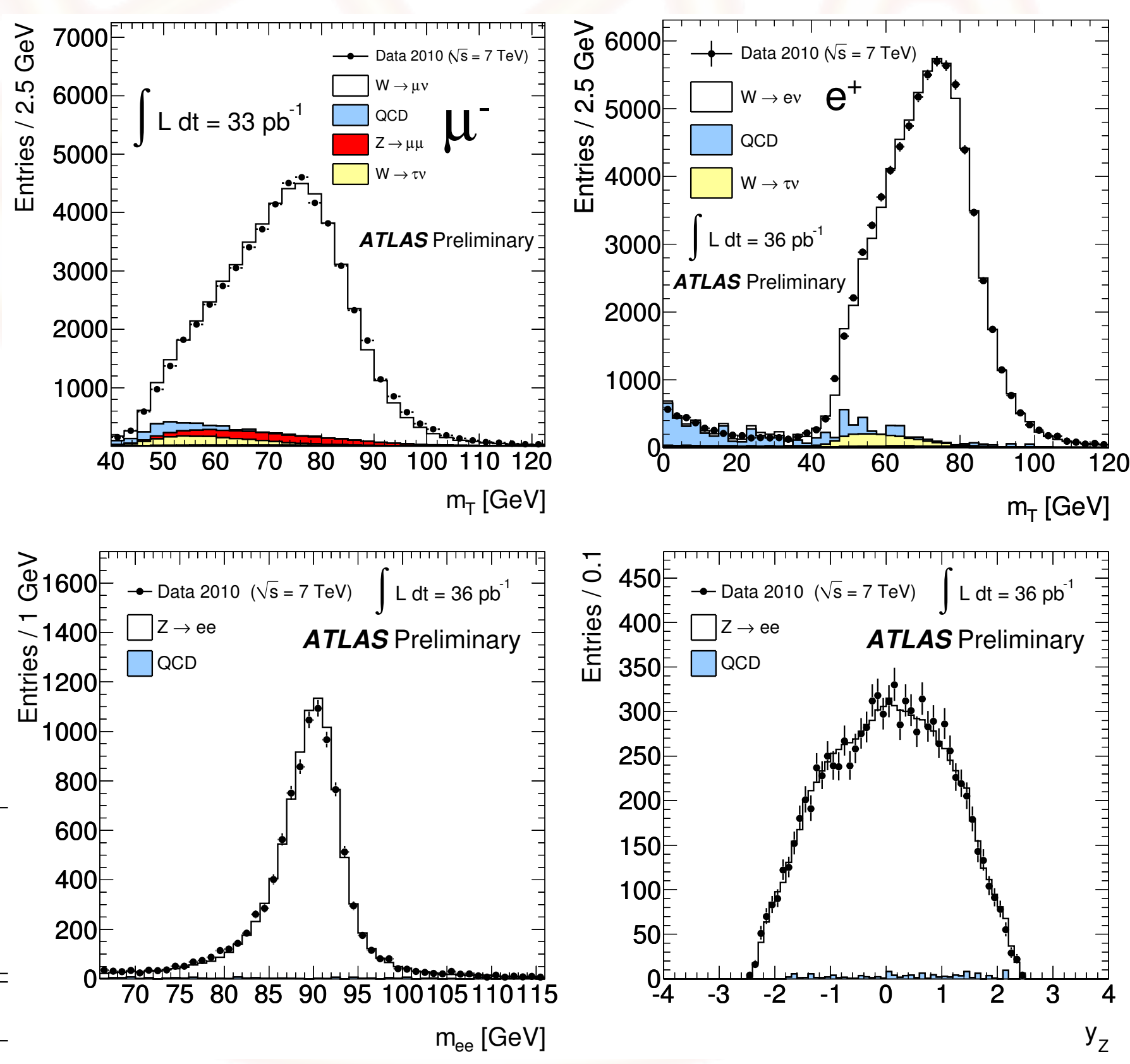

ATLAS-CONF-2011-041 


\section{W/Z cross section ratios}

- Systematic uncertainties

- luminosity: $3.4 \%$

- acceptance corrections: $3-4 \%$

- $\mathrm{E}_{T}{ }^{\text {miss }}$ requirement: $2 \%$

- electron ID: I-2\%
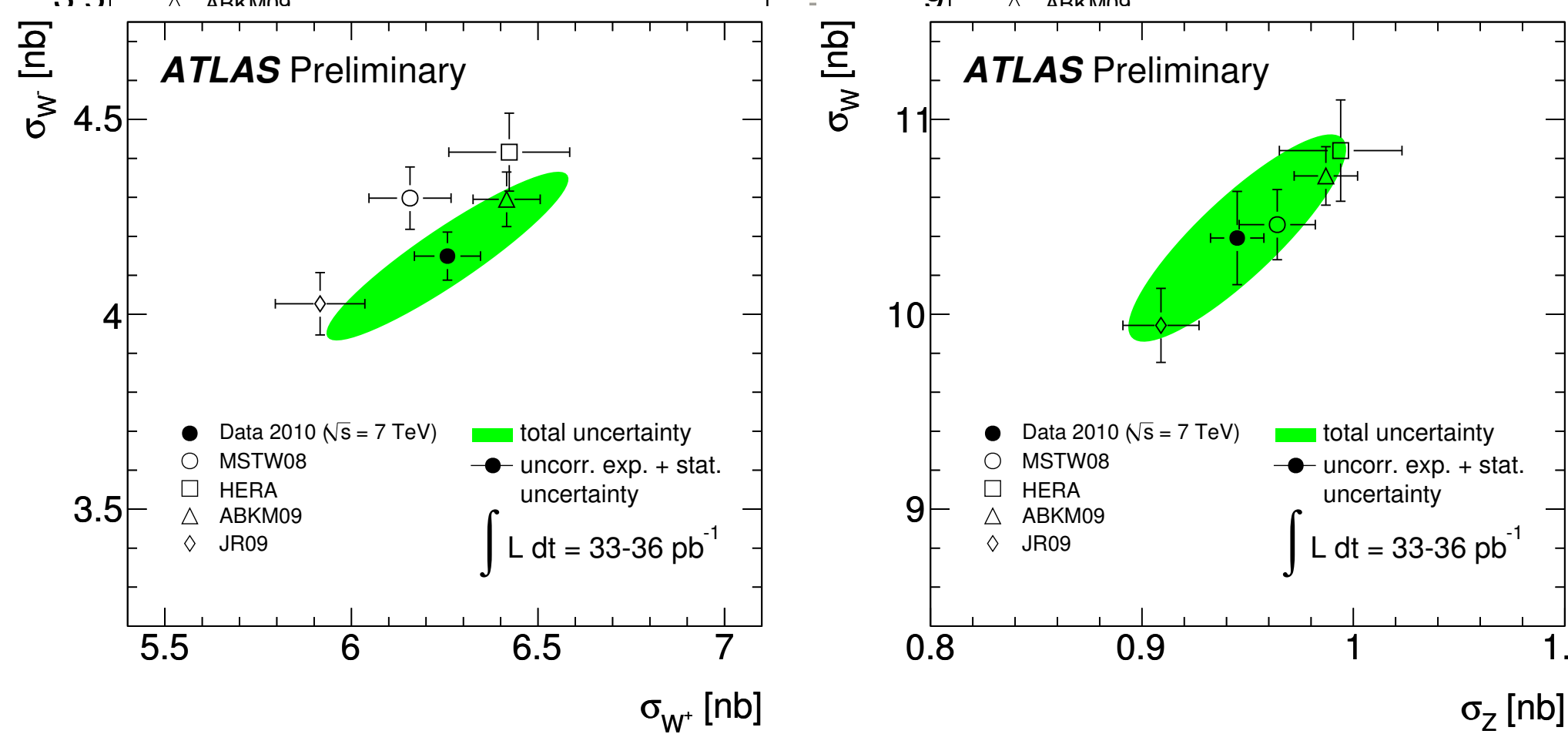

- Results

- good agreement with theoretical prediction in NNLO QCD

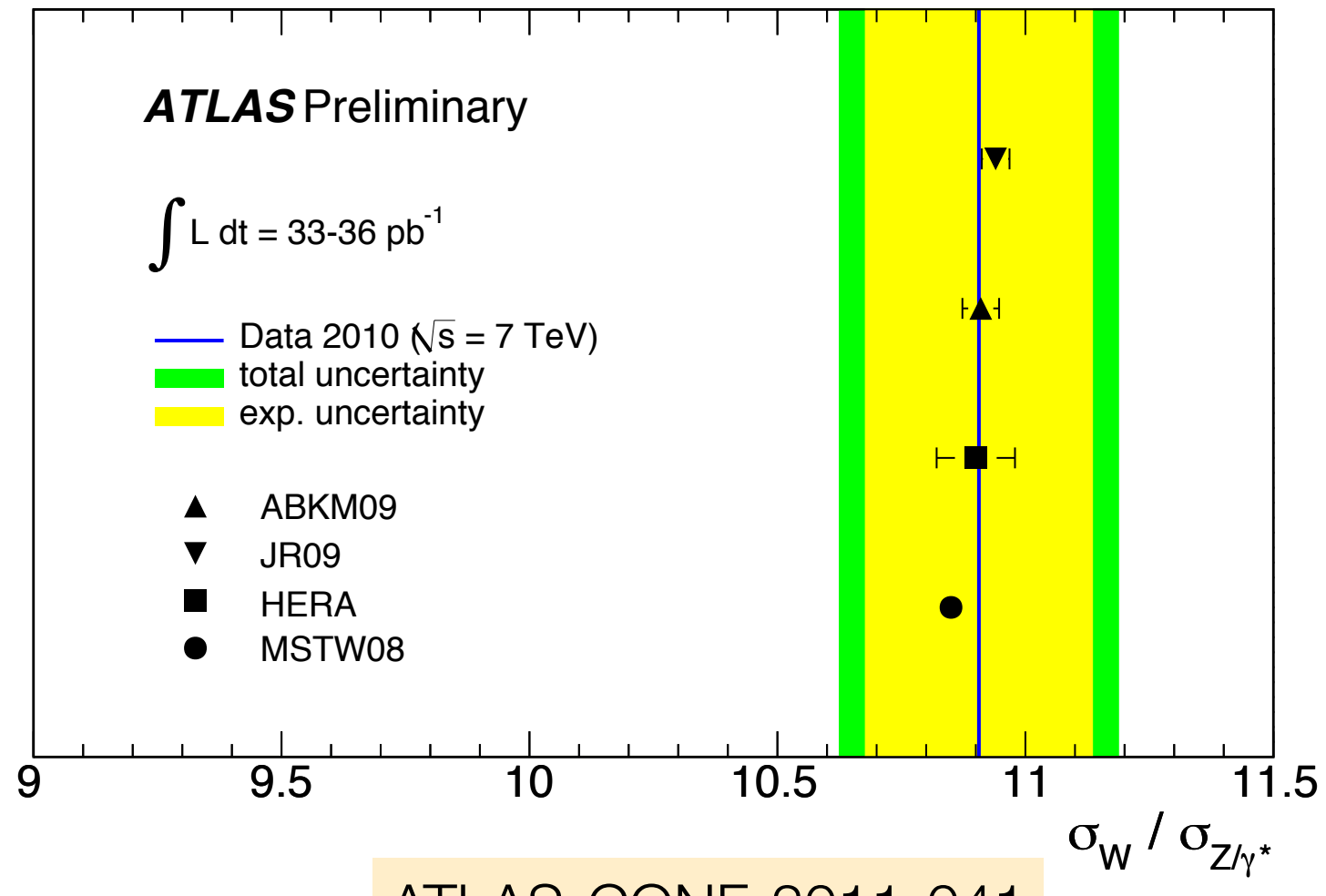

ATLAS-CONF-2011-041 


\section{$W+$ jets}

- Motivation of $W(Z)+$ jets measurements

- very powerful tool to test PQCD

- important background for Higgs/SUSY searches

- Signal selection

- leptons: similar to inclusive measurement

- jet definition

- anti-kT with $\mathrm{R}=0.4$

- $\left|y_{\text {jet }}\right|<2.8$

- $\mathrm{pT}>20 \mathrm{GeV}$

- lepton/jet overlap removal - $\Delta R<0.5$

- pile-up jets are rejected by jet-vertex-fraction (JVF):

- $\Sigma \mathrm{p} T$ (tracks) from other primary vertices

- Main backgrounds

- QCD

- top : electron channel

- Dominating systematic

- JES : 10-20\%

- Results

- very good agreement between data and $\mathrm{PQCD}$ predictions

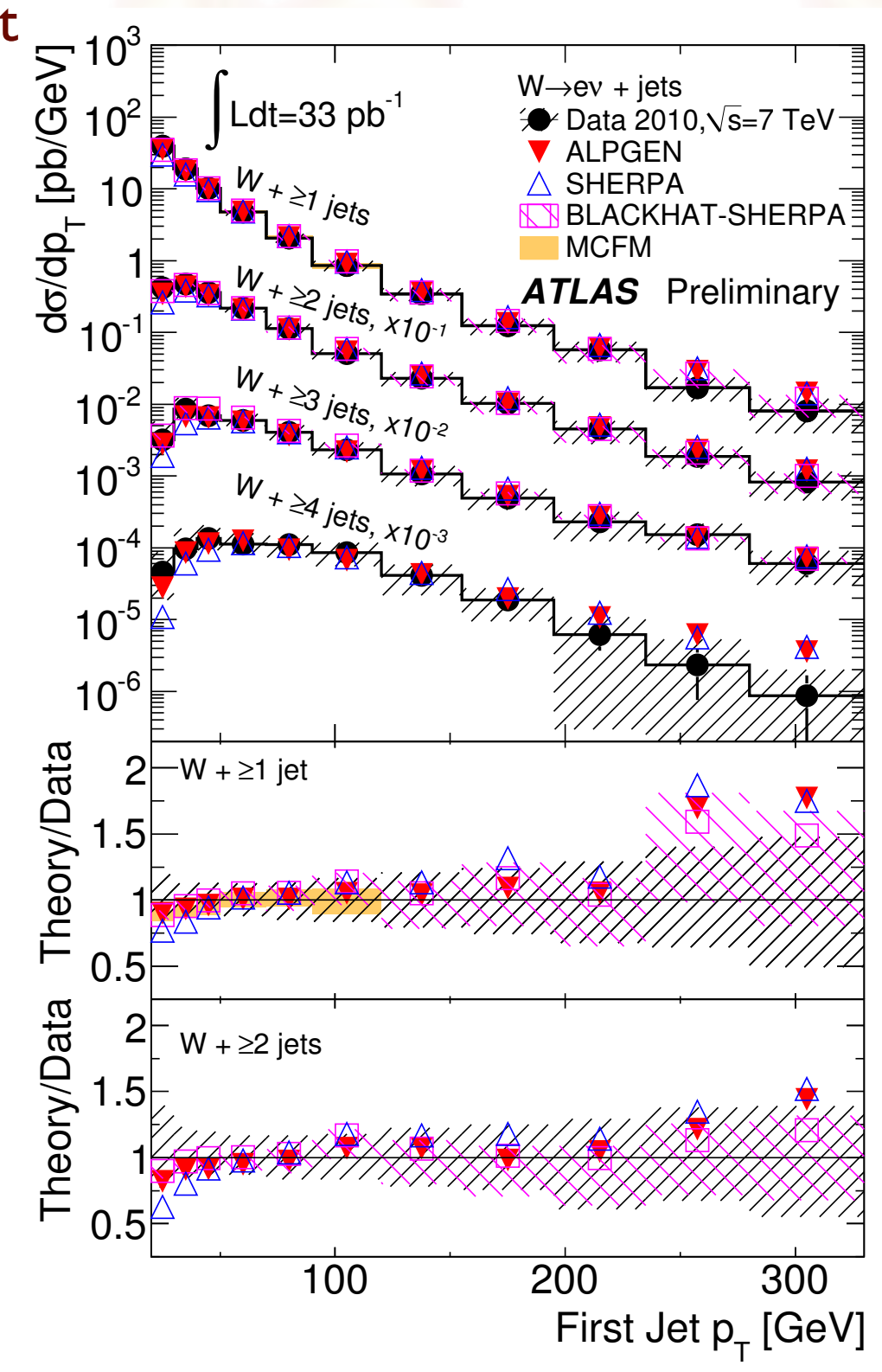

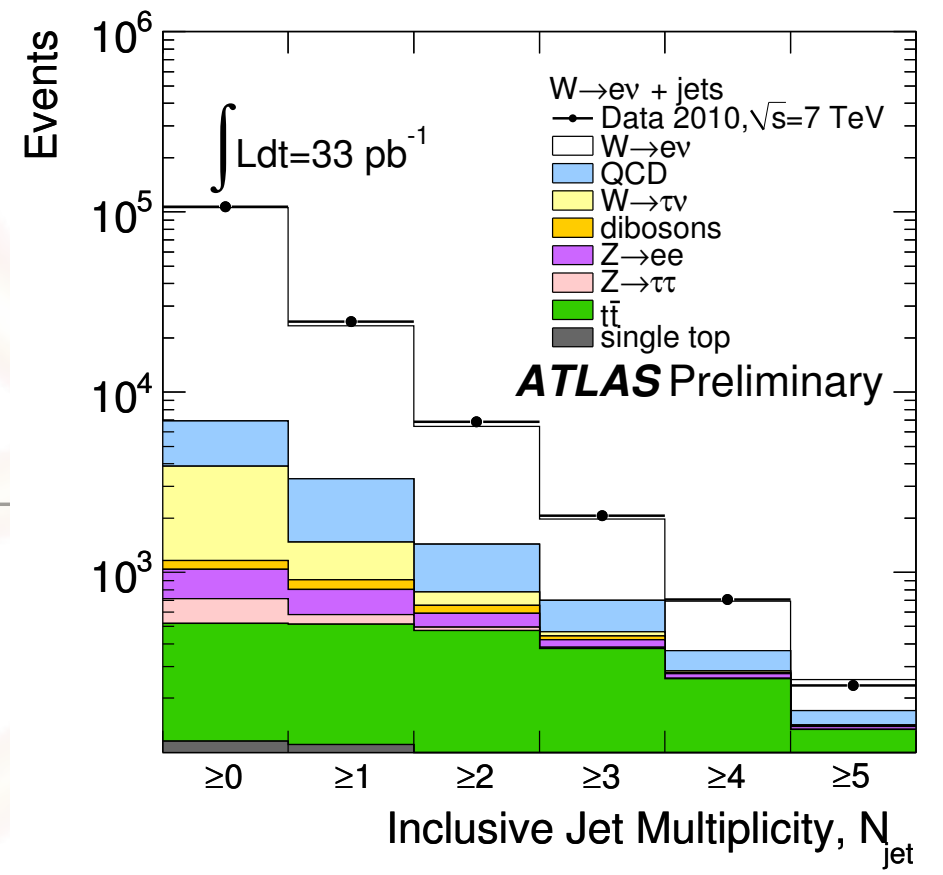
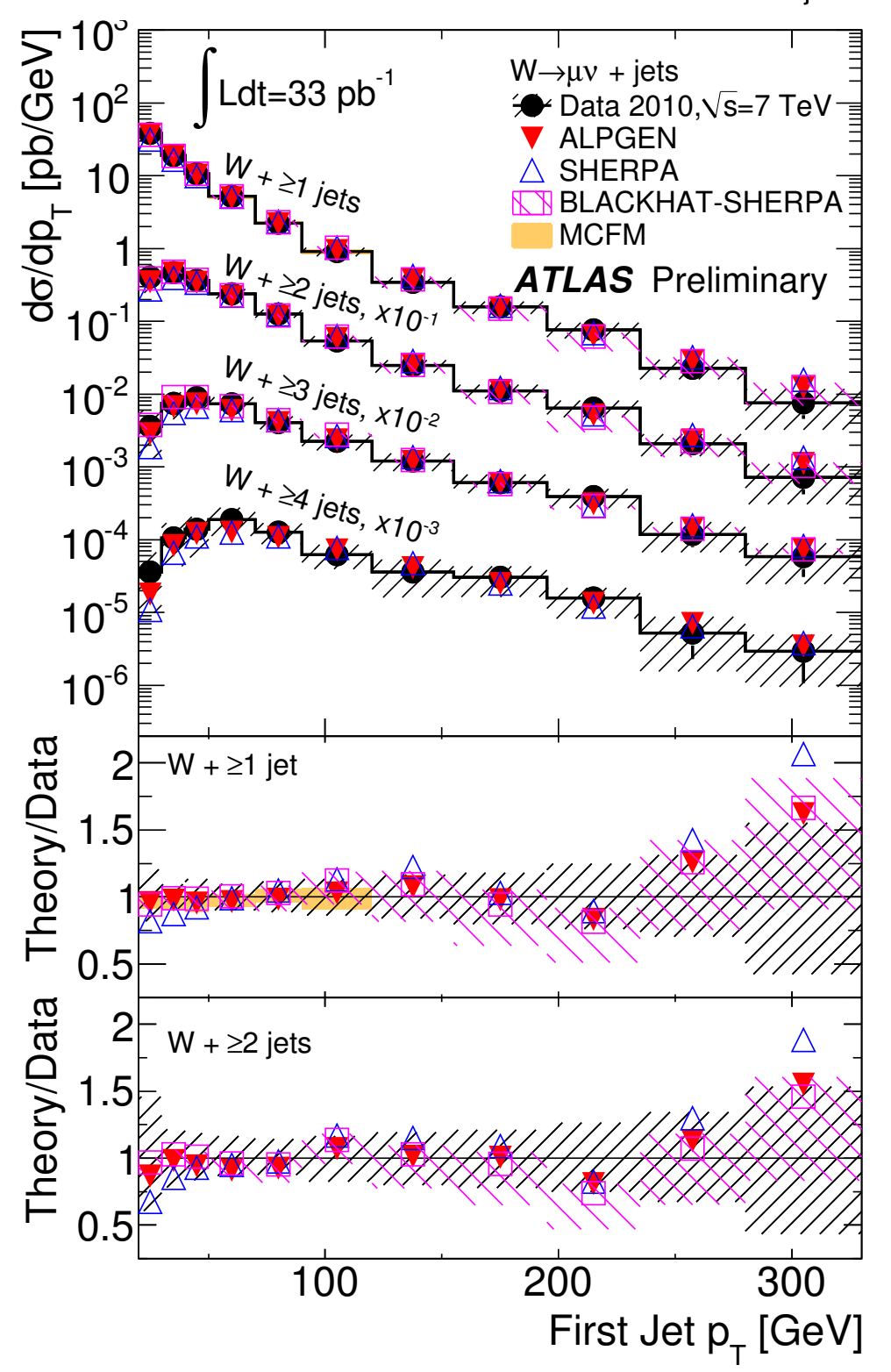

ATLAS-CONF-2011-060

Toshi SUMIDA

2 


\section{W production}
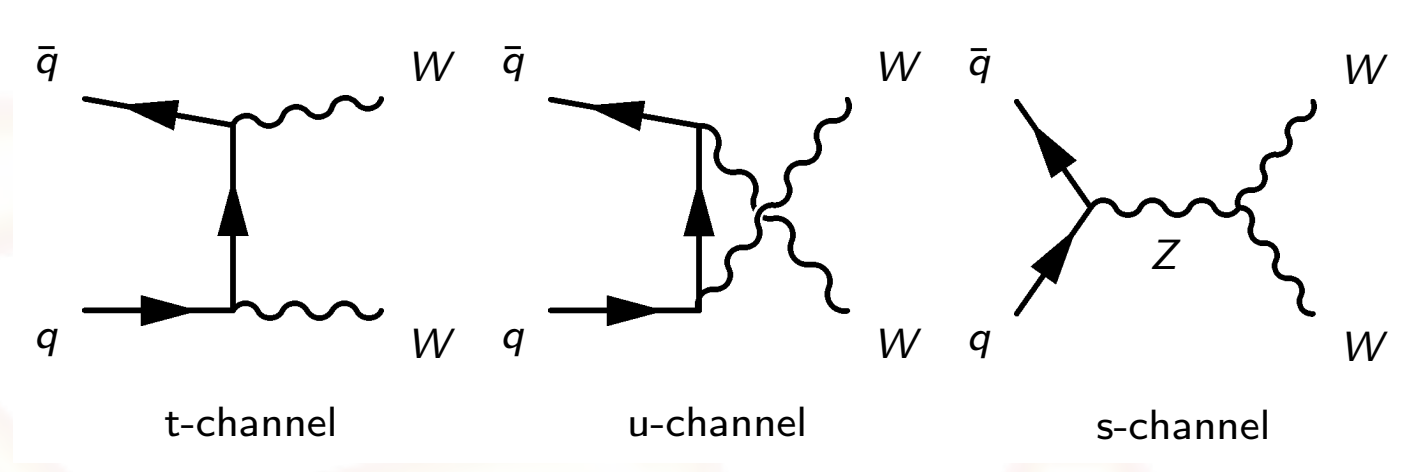

- Signal selection

- PT(lepton) > $20 \mathrm{GeV}$

- $\mathrm{E}_{\mathrm{T}}^{\mathrm{miss}}$

- $>40 \mathrm{GeV}$ ee/ $\mu \mu$

- $>20 \mathrm{GeV}$ er

- Veto $\left|m_{\| 1}-m_{z}\right|<10 \mathrm{GeV}$

- Veto jets with рт(jet) $>20 \mathrm{GeV}$ in $|\eta|<3$

- Backgrounds

- Drell-Yan + fake $E_{T}{ }^{\text {miss }}$

- other dibosons

- W+jets with a jet faking a lepton

- tt-bar with two lost b-jets

- Results in 2010 data

- statistically dominated

- 8 events observed

- expectation

$\checkmark 6.85$ signal

- p-value

$$
\checkmark 1.68 \mathrm{BG}
$$

- $1.72 \pm 0.37 \pm 0.45: 3 \sigma$
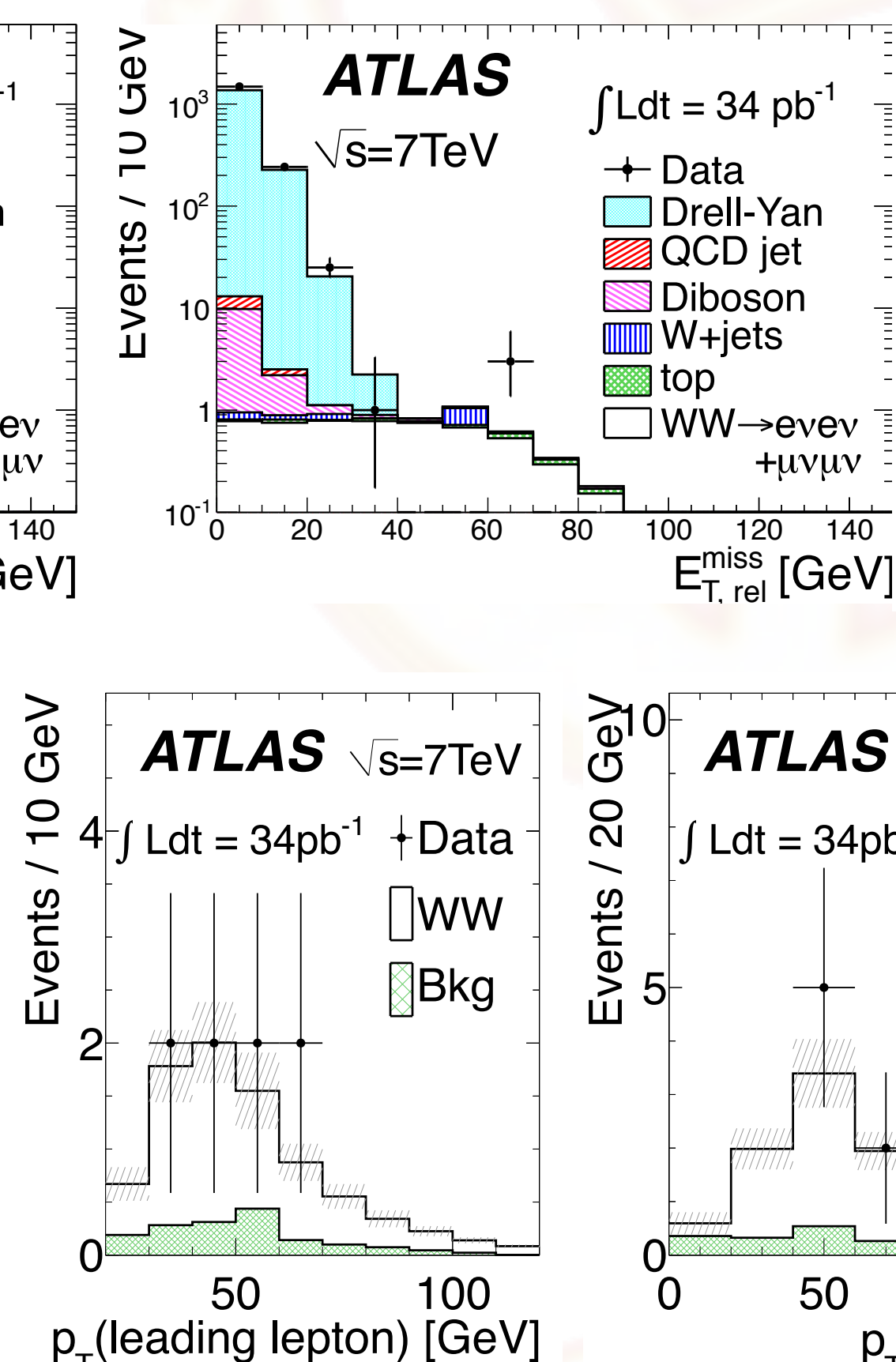

- $\sigma_{w w}=4 I^{+20}{ }_{-16}$ (stat.) \pm 5 (syst.) $\pm \mathrm{I}$ (lumi.) pb

- prediction: $44 \pm 3$ (syst.) pb
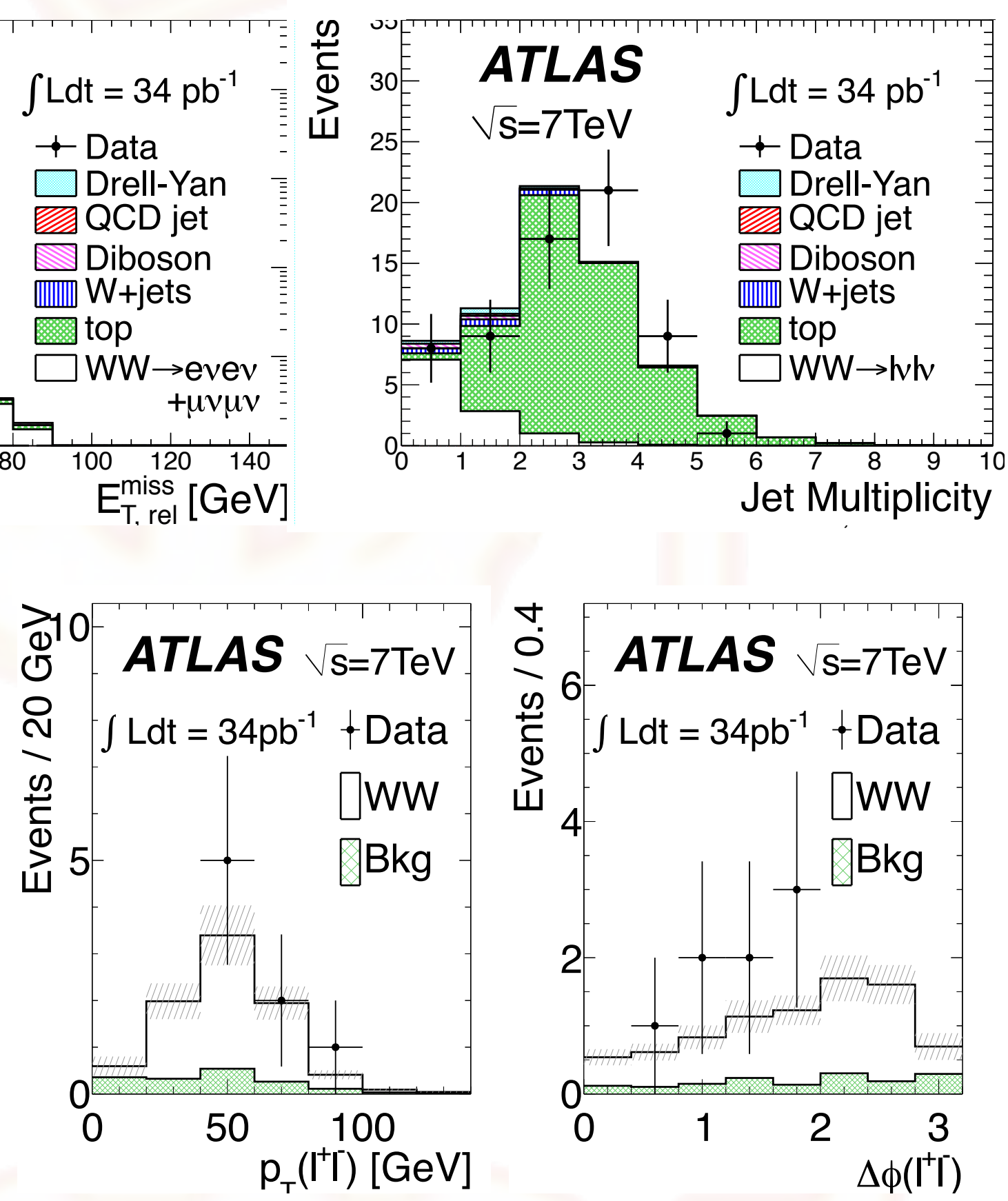

arXiv: 1104.5225, submitted to PRL 


\section{WZ production}

- Signal selection

- at least 3 leptons, $Z$ candidate two leptons with

- $\left|M_{\|}-M_{Z}\right|<10 G e V$

- $\mathrm{E}_{T}$ miss $>25 \mathrm{GeV}$

- W transverse mass $M_{T}\left(l_{3}, E_{T}\right)>20 G e V$

- Main background sources

- 3 hard leptons mainly from ZZ events

- 2 hard leptons (Z,WW, top) + a 3rd one coming from a jet faking a lepton

- heavy quark decay, decay in flight

- I hard lepton and 2 leptons from fake or heavy flavor decay

- Results with $205 \mathrm{pb}^{-1}$ in $201 \mathrm{I}$ data

- $\sigma_{W z^{\text {tot }}}=18^{+7}$-6 (stat.) \pm 3 (syst.) $\pm I$ (lumi.) pb

- prediction: $16.9^{+1.2}-0.8$ (syst.) pb

$\checkmark$ good agreement with the SM expectation
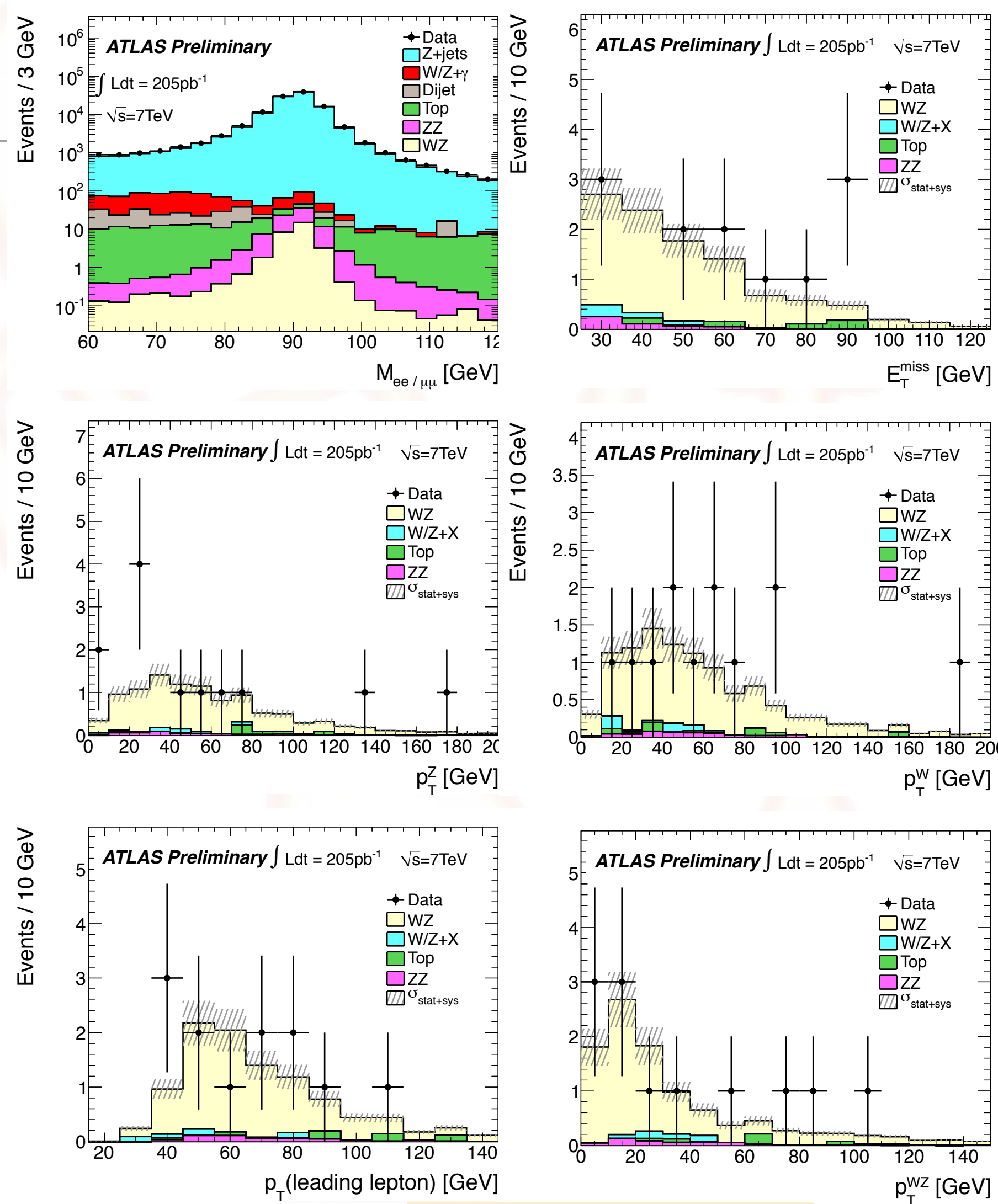

ATLAS-CONF-2011-084 


\section{Summary of W/Z production}

- ATLASW and $Z$ results with the 2010 dataset $\left(3 I-35 \mathrm{pb}^{-1}\right)$

- Conclusions

- important tests of $p Q C D$, constraints for PDFs

- $\mathrm{W}$ and $\mathrm{Z}$ bosons have been observed using electron, muon and tau channels

- demonstrated good performance and understanding of the detecter

- results show good agreement with SM expectations

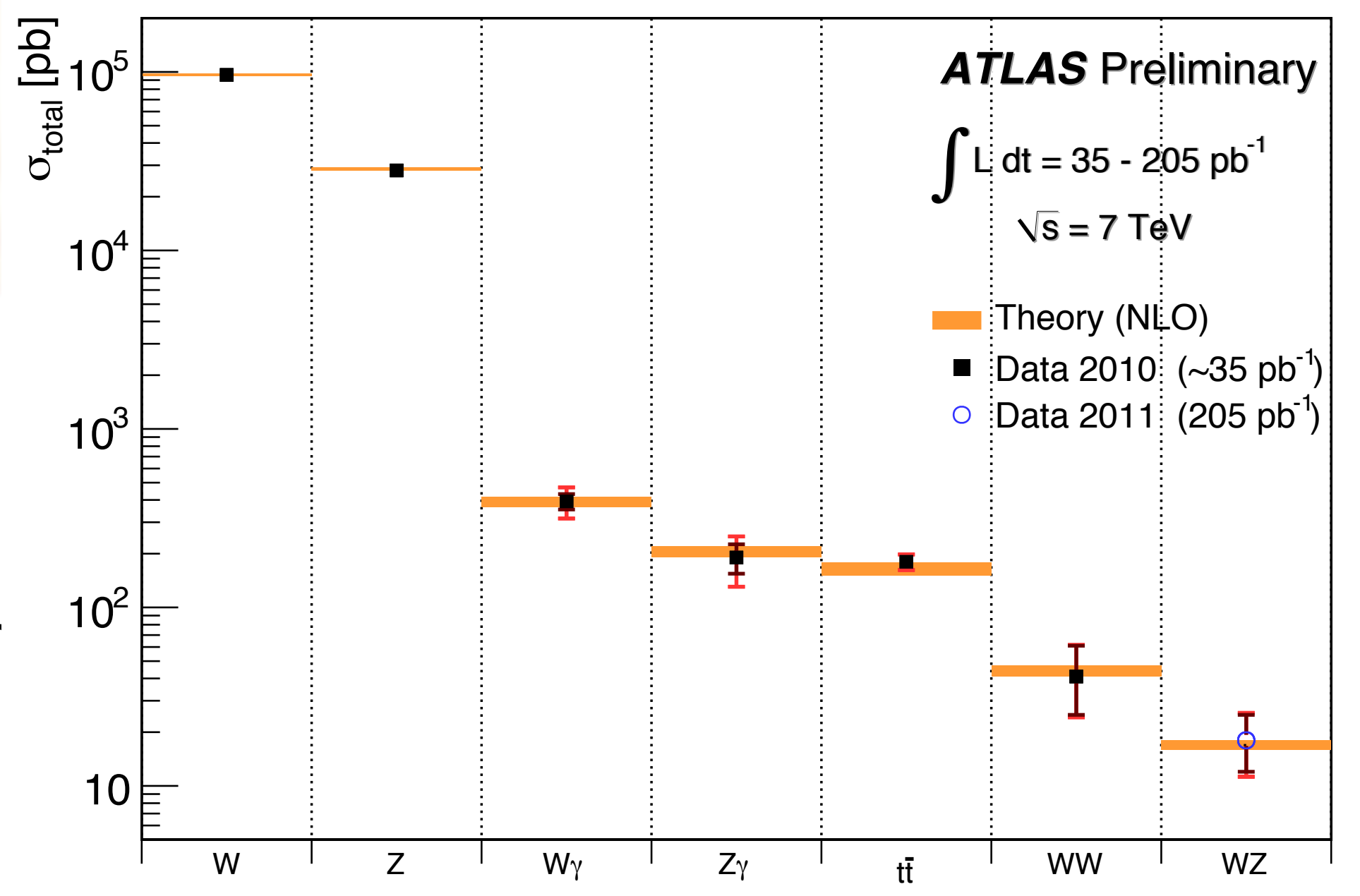

- 20 II data

- dramatically improves statistics, and allows precision measurements

- W/Z already measured in $201 \mathrm{I}$ data 


\section{Top quark}

(

(1)

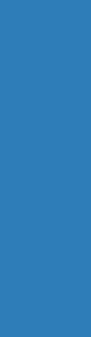




\section{Top events}

- Top decay in the SM

- $\operatorname{Br}(\mathrm{t} \rightarrow \mathrm{Wb}) \approx 100 \%$

- Challenging signature

- multiple leptons \& jets

- missing $\mathrm{E}_{\mathrm{T}}$

- $(\mathrm{t} \overline{\mathrm{t}})$ decay signatures characterized

by $\mathrm{W}$ decays

- all-Hadronic

- $\mathrm{Br} \sim 45 \%$

- large QCD background

- no significance yet

- lepton + jets

- $\mathrm{Br} \sim 30 \%$

- moderate backgrounds

- dilepton

- $\mathrm{Br} \sim 5 \%$

- very clean

- Dominant backgrounds for leptonic channels

- W/Z + jets

- QCD jets

- Signal

- event selection

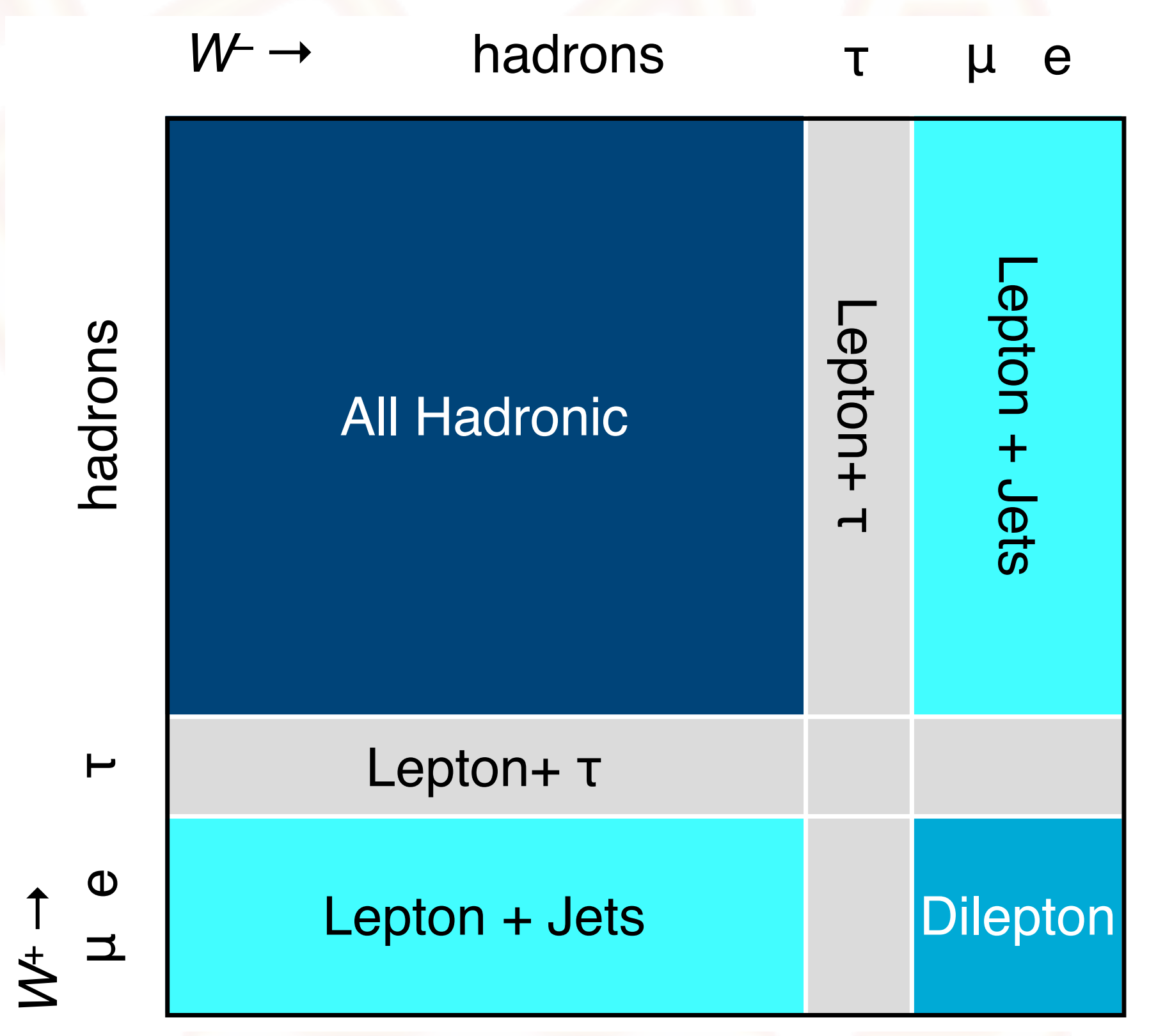

- high-pT leptons, $\geq 3$ jets, $\mathrm{E}_{\mathrm{T}}^{\text {miss }}$, transverse mass

- multi-region fit : $(e, \mu)+$ njets 


\section{Top results}

- Top pair production

- single lepton

- dilepton

- summary of cross section measurements

- Top quark mass measurement

-W polarization in top decays

- Single top production 


\section{Top results}

- Top pair production

- single lepton

- dilepton

- summary of cross section measurements

- Top quark mass measurement

- Single top production 


\section{Top pair production}

- single lepton + jets

- analysis I: without b-tagging

- fit of projective likelihood based on uncorrelated variables

- lepton $\eta$, lepton charge, ...

analysis 2: with "continuous" b-tagging

- profile likelihood fit extracts 16 parameters including $\sigma_{\mathrm{tt}}$

- Results in 2010 data

- without b-tagging

- $\sigma_{\mathrm{tt}}=17 \mid \pm 17$ (stat) ${ }^{+20}{ }_{-17}$ (syst) \pm 6 (lumi) pb

- with b-tagging

- $\sigma_{\mathrm{tt}}=186 \pm 10$ (stat) ${ }^{+2 \mathrm{I}}{ }_{-20}$ (syst) \pm 6 (lumi) $\mathrm{pb}$

- Uncertainty

- JES dominated: I3-15\%

- improvements expected in 20II

ATLAS-CONF-2011-023 ATLAS-CONF-2011-035
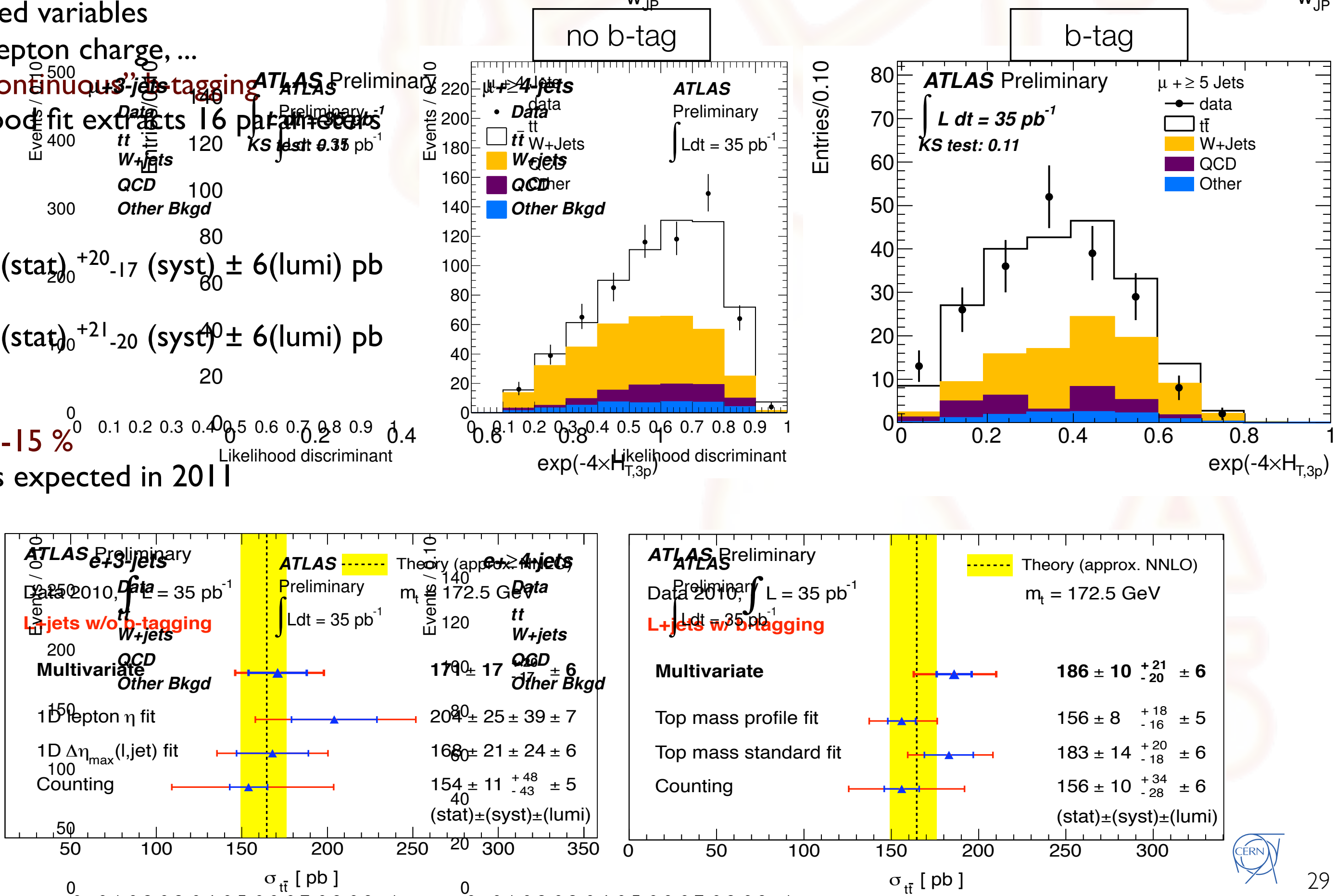

$\sigma_{\mathrm{tt}}[\mathrm{pb}]$

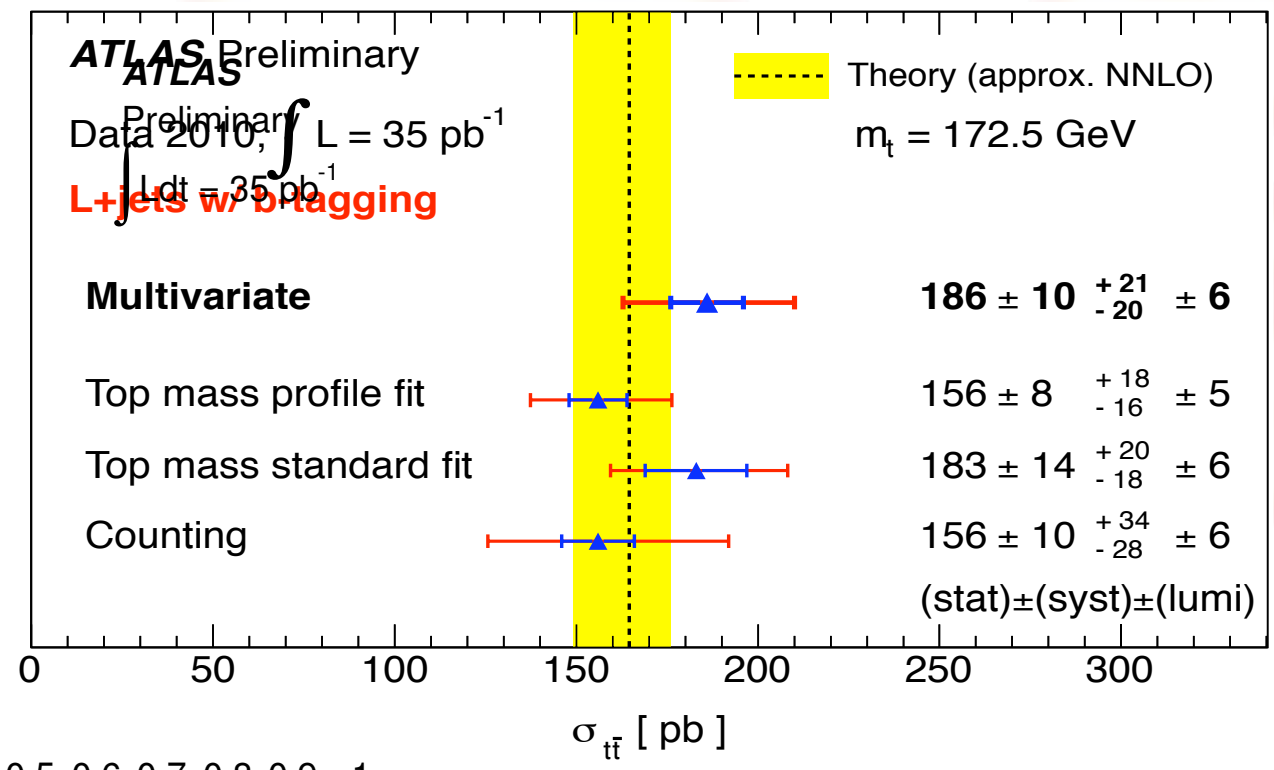




\section{Top cross section}

- Combined result in 2010 data

- lepton + jets and dilepton analyses on the level of likelihood functions

- $\sigma_{\mathrm{tt}}=180 \pm 9$ (stat.) \pm 15 (syst.) \pm 6 (lumi.) $\mathrm{pb}$
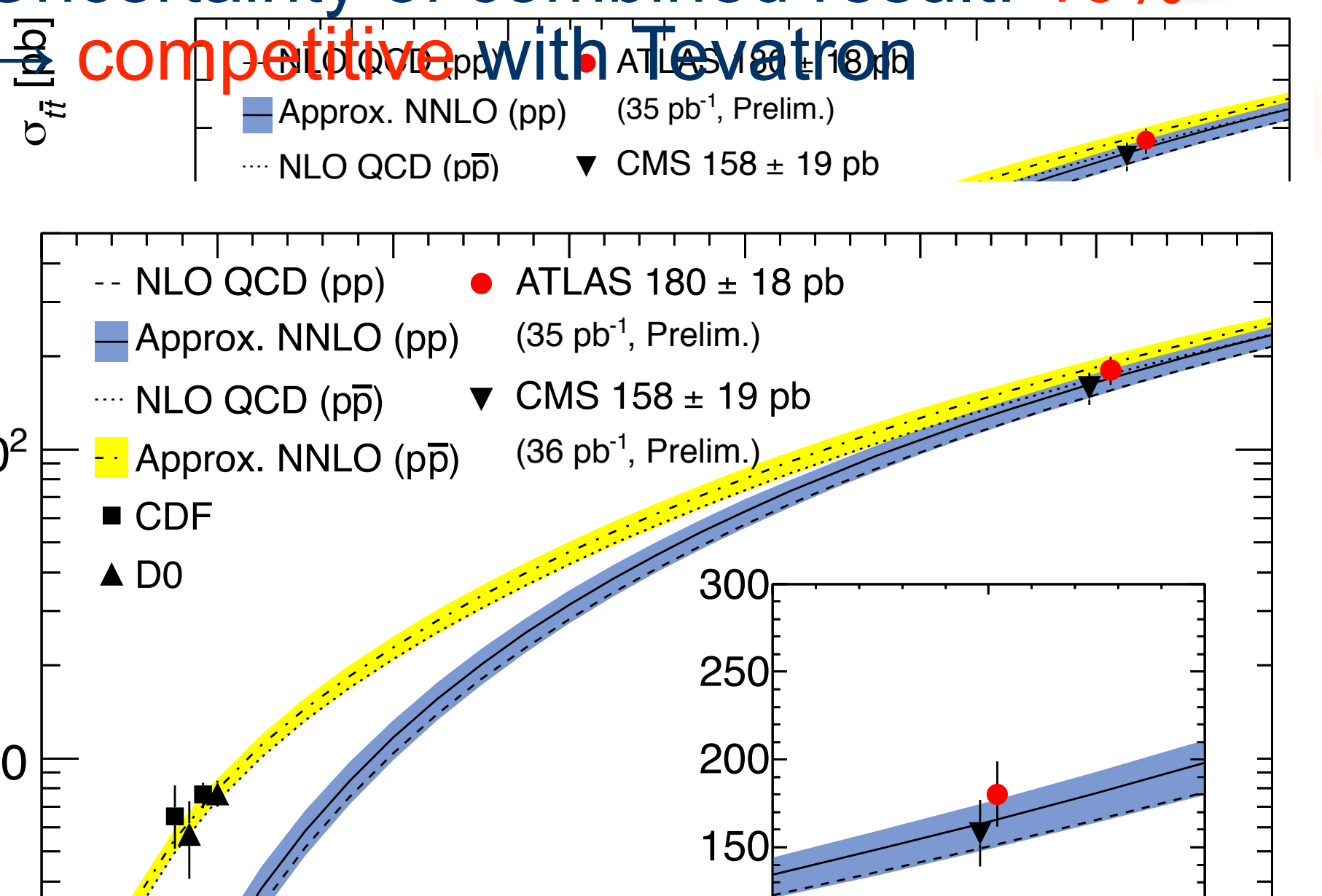

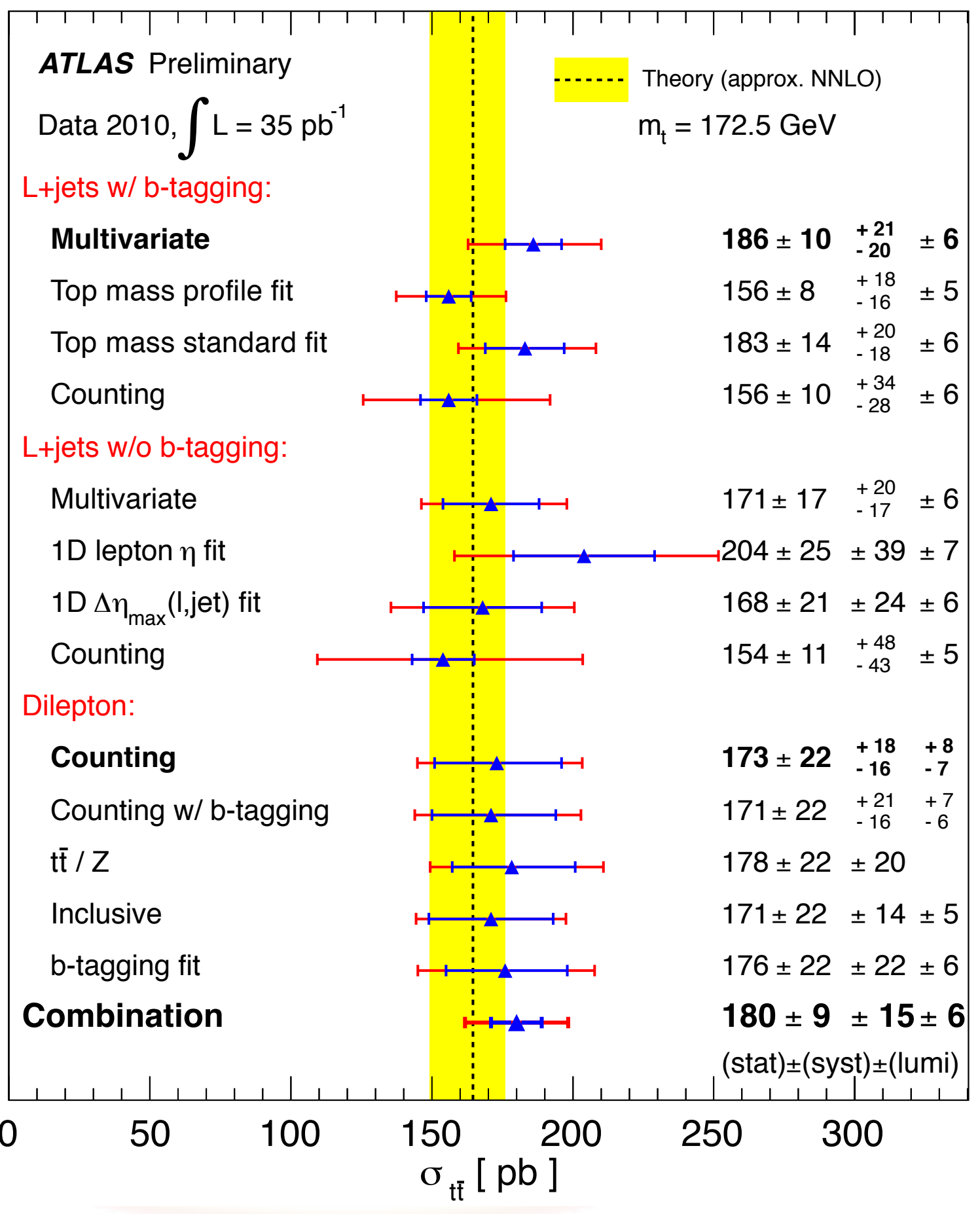

ATLAS-CONF-2011-040 


\section{Top quark mass measurement}

- Direct measurement

- top mass: key parameter in SM

- high precision from Tevatron $(0.6 \%)$

- 2010 data $\left(35 p^{-1}\right)$

- template-based analyses

- Lepton + jets selection

- ID “stabilized mass": $R_{32}=m_{t}^{\text {reco }} / m_{w^{\text {reco }}}$

- ID kinematic fit

- 2D: mass vs. Jet energy scale factor (JSF)

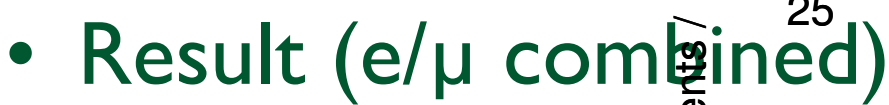

- $m_{t}=169.3 \pm 4.0$ (stat.) \pm 4.9 (syst.) $\mathrm{GeV}$

- $3.7 \%$ total uncertainty

$\checkmark$ cf.) Tevatron $172 \pm 0.9 \pm 1.3 \mathrm{GeV}$
1D stabilized mass ( $\mu+$ jets)
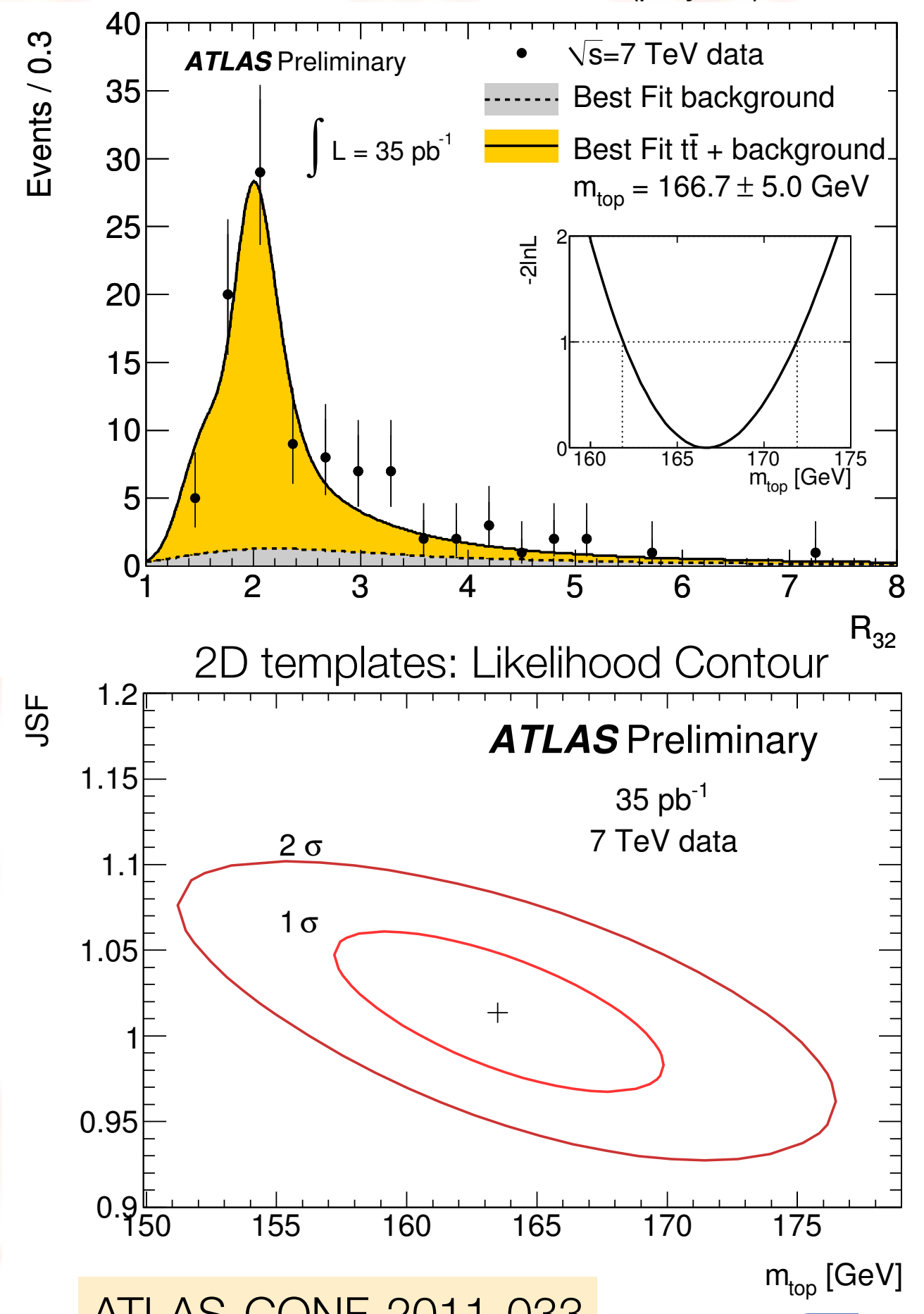

ATLAS-CONF-2011-033 


\section{Single top production}

- t-channel production analysis

- $156 \mathrm{pb}^{-1}$ of 20I I ATLAS data

- Lepton + jets final state

- Cut-based and

Neural network analysis

- Cross section

- cut based:

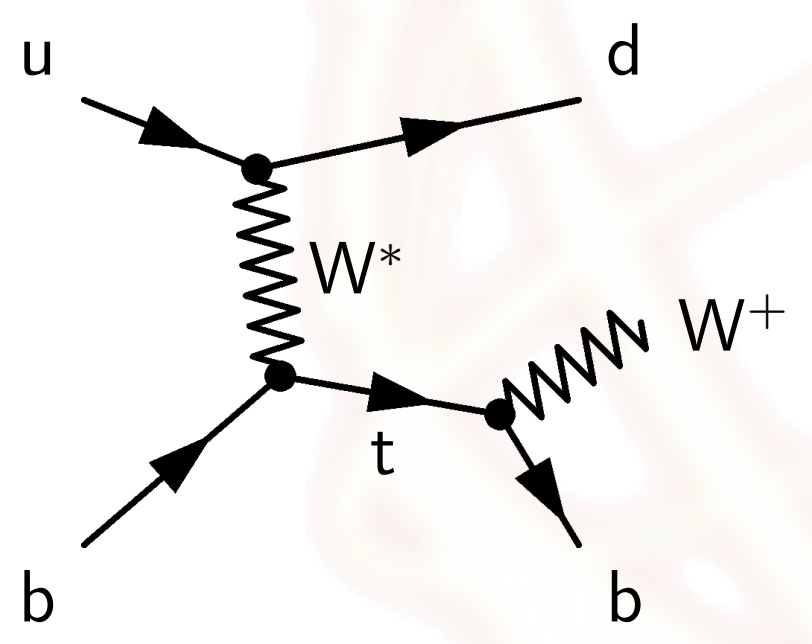

- $\sigma_{\mathrm{t}}=97^{+56}-30 \mathrm{pb}$

- NN based:

- $\sigma_{\mathrm{t}}=76^{+41}-21 \mathrm{pb}$

$\checkmark \sigma_{\mathrm{t}}(\mathrm{SM})=66 \mathrm{pb}$

- observed (expected) significance

- cut based

- $6.2(5.7) \sigma$

- NN based

- $6.3(4.5) \sigma$

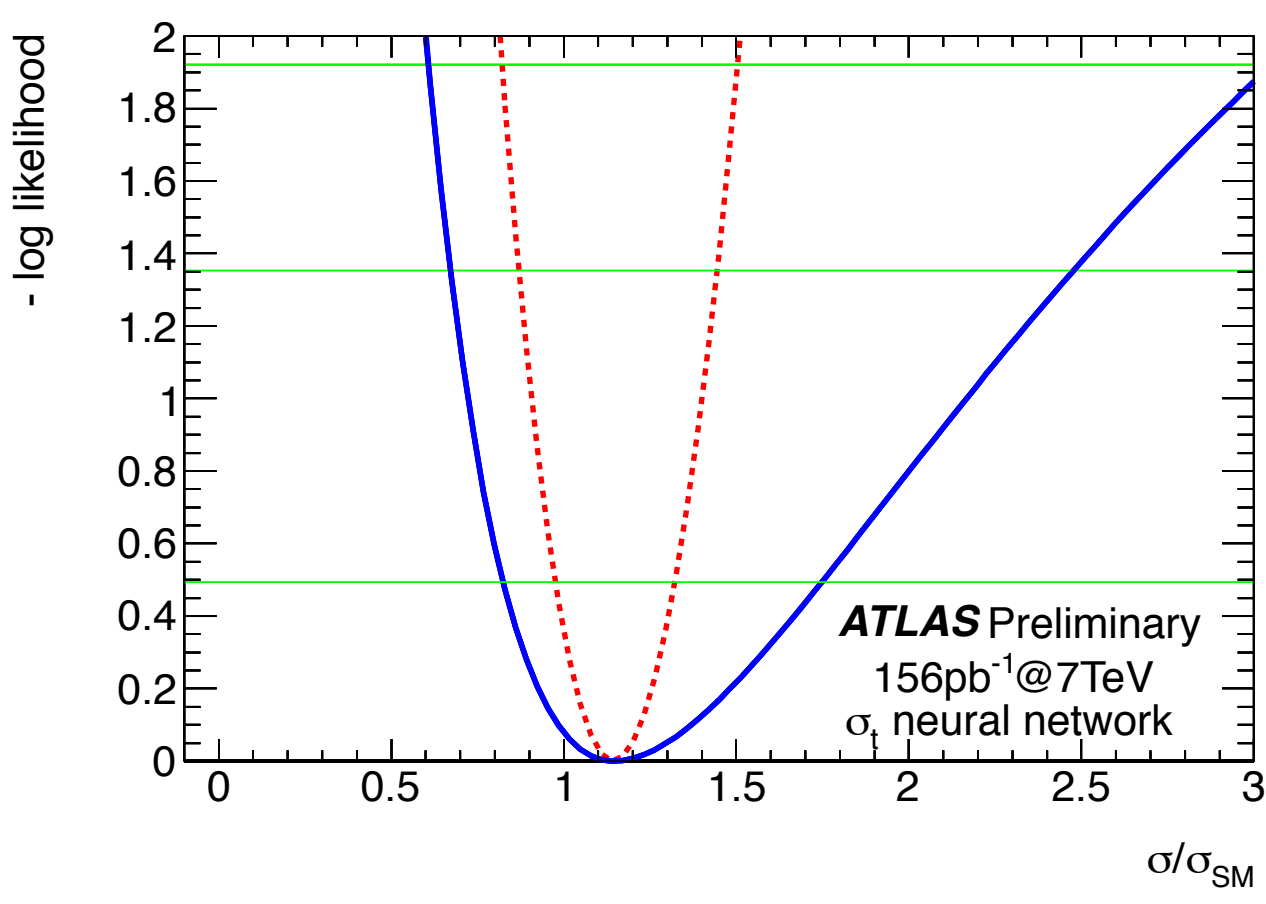

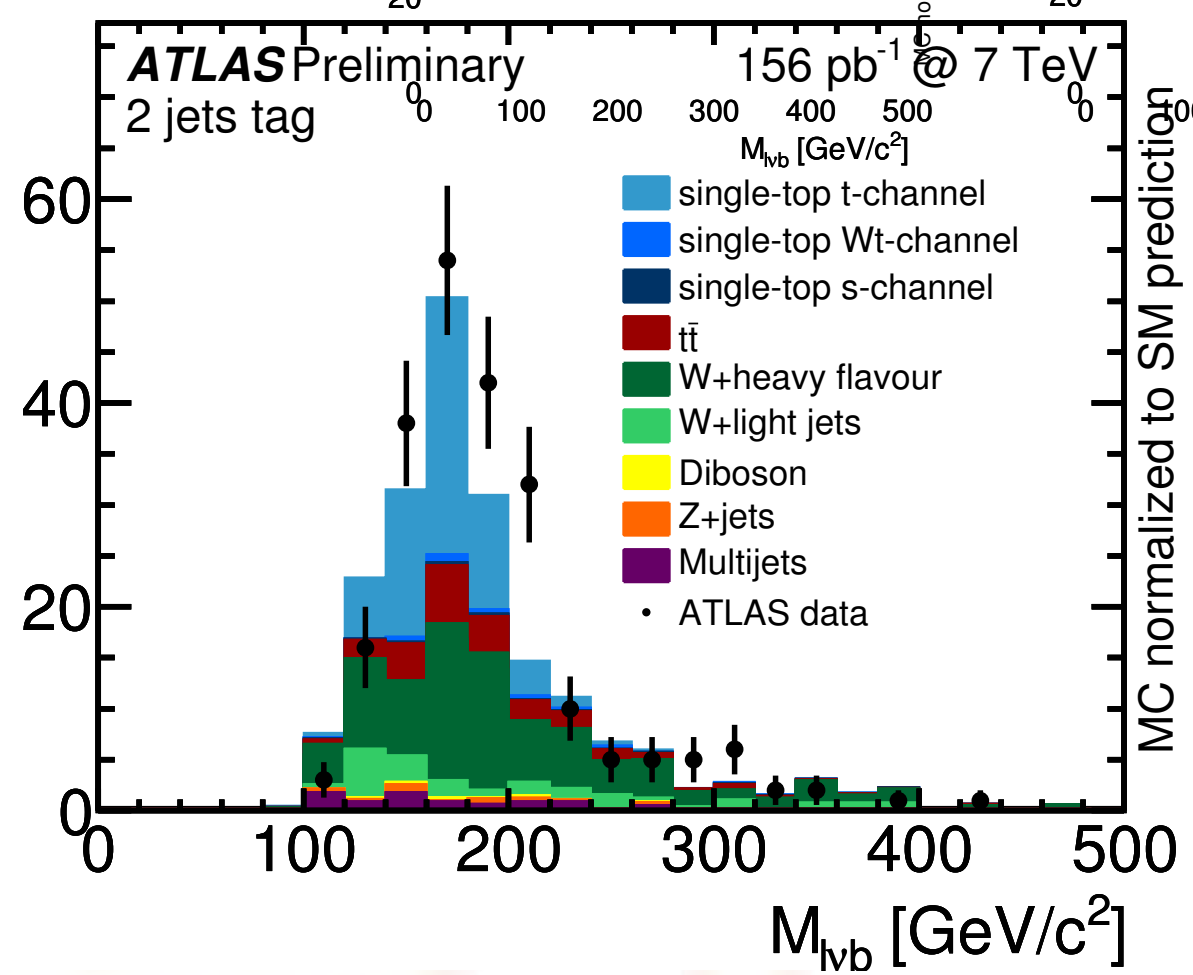

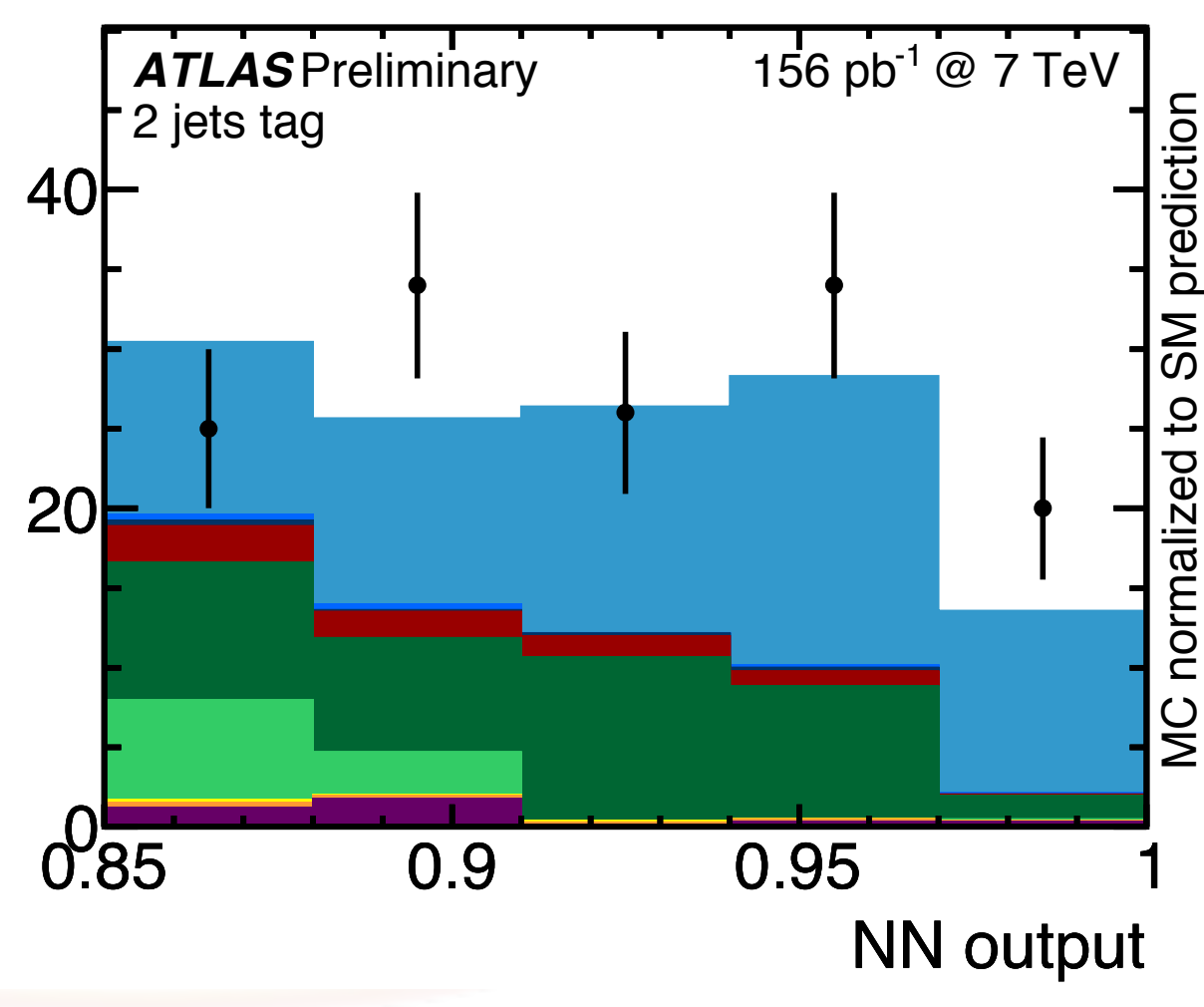

Toshi SUMIDA
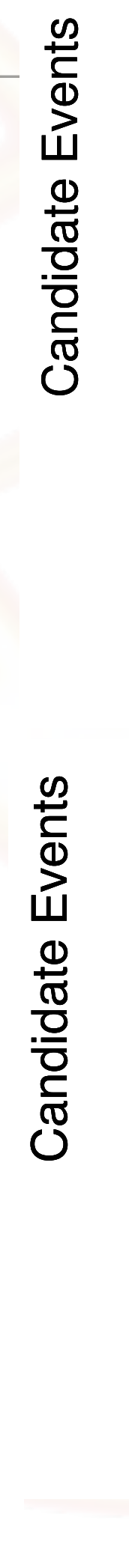


\section{Summary}

- LHC

- working extremely well

- delivered more than $\mathrm{I} \mathrm{fb}^{-1}$ in $20 \mathrm{II}$

- ATLAS

- taking very good quality data

- data analysis is proceeding at very high speed

- Standard model results presented by ATLAS

- high-pт jets

- probe QCD to a new energy regime and larger rapidities

- electro weak bosons

- important tests of $\mathrm{PQCD}$

- top quark

- cross section

- mass and properties

- The overall standard prediction with PQCD shows good agreement with $\sqrt{s}=7 \mathrm{TeV}$ data

- results with $200 \mathrm{pb}^{-1}$ shown

- We will have more precise SM measurements in 20II/20I2 with 2 - $10 \mathrm{fb}^{-1}$ of integrated luminosity!! 


\section{Luminosity calibration}

- Total 5 Van der Meer scans ( I-V ) in 2010

- Previous error on luminosity calibration from the first three scans: 1 1\%

- Dominated by uncertainty on bunch current (10\%)

- Understanding the beam current in the scan IV,V

- transformers (BCTs) and improve measurement

\begin{tabular}{lccc}
\hline \hline Scan Number & I & II-III & IV-V \\
Fill Number & 1059 & 1089 & 1386 \\
\hline DCCT baseline offset & $3.9 \%$ & $1.9 \%$ & $0.1 \%$ \\
DCCT scale variation & $2.7 \%$ & $2.7 \%$ & $2.7 \%$ \\
Bunch-to-bunch fraction & $2.9 \%$ & $2.9 \%$ & $1.0 \%$ \\
\hline Total & $5.6 \%$ & $4.4 \%$ & $2.9 \%$ \\
\hline \hline
\end{tabular}

- Result

- $\sigma v i s=41.75 \pm 1.30 \mathrm{mb}$

- Total uncertainty: $3.2 \%$

- significant improvement

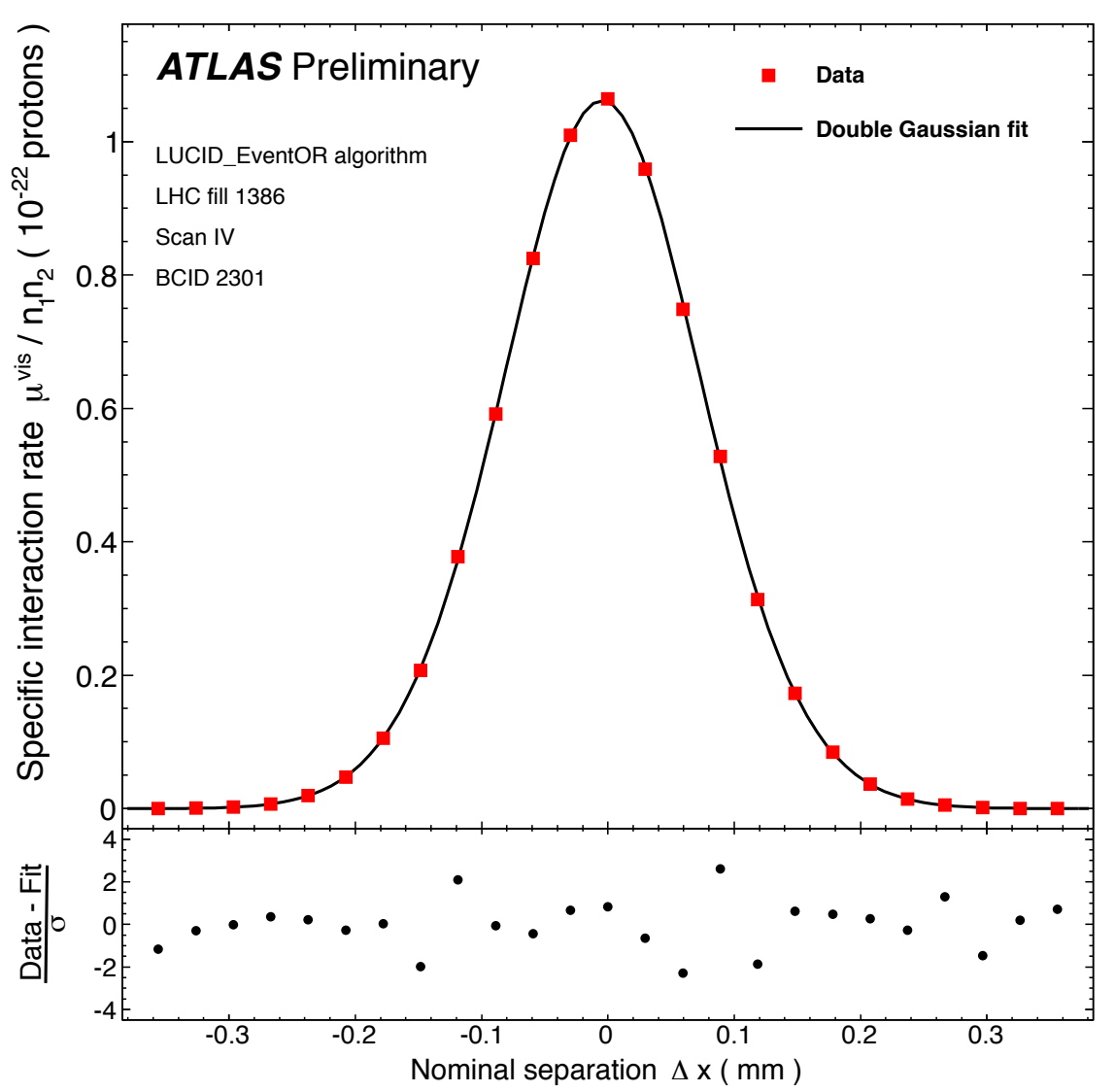

\begin{tabular}{lc}
\hline \hline Uncertainty Source & $\delta \mathcal{L} / \mathcal{L}$ \\
\hline Statistical & $0.1 \%$ \\
Bunch charge product & $2.9 \%$ \\
Beam centering & $<0.1 \%$ \\
Emittance growth and & \\
other non-reproducibility & $0.3 \%$ \\
Beam position & \\
jitter & $0.2 \%$ \\
Length scale calibration & $0.3 \%$ \\
Absolute ID length scale & $0.3 \%$ \\
Fit model & $0.2 \%$ \\
Transverse correlations & $0.9 \%$ \\
$\mu$ dependence & $0.5 \%$ \\
\hline Long-term consistency & $0.5 \%$ \\
\hline Total & $3.2 \%$ \\
\hline \hline
\end{tabular}




\section{Inner Detector}

- Purpose

- to measure charged tracks trajectories in a 2 Tesla SC solenoidal B-field

- 3 sub-detectors: (Resolution)

- Pixel: I0/II5 $\mu \mathrm{m}$ in RФ/z

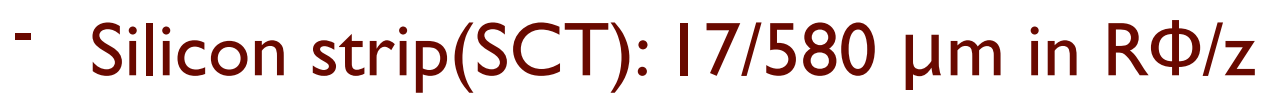

- Transition Radiation Tracker (TRT): $130 \mu \mathrm{m}$ in $\mathrm{R} \Phi$

- Coverage

- $|\eta|<2.5$ (2.0 for TRT) with 3 Pixel measurements, 8 SCT and 230 TRT

- Design

- $\varepsilon_{\text {track }}>90 \%(\pi)$ and $99 \%(\mu)$

- $\sigma_{\mathrm{PT}} / \mathrm{PT}=0.05 \%$ PT $\oplus \mathrm{I} \%$

- $\sigma(\mathrm{ip})=10 \mu \mathrm{m}$ at high momentum

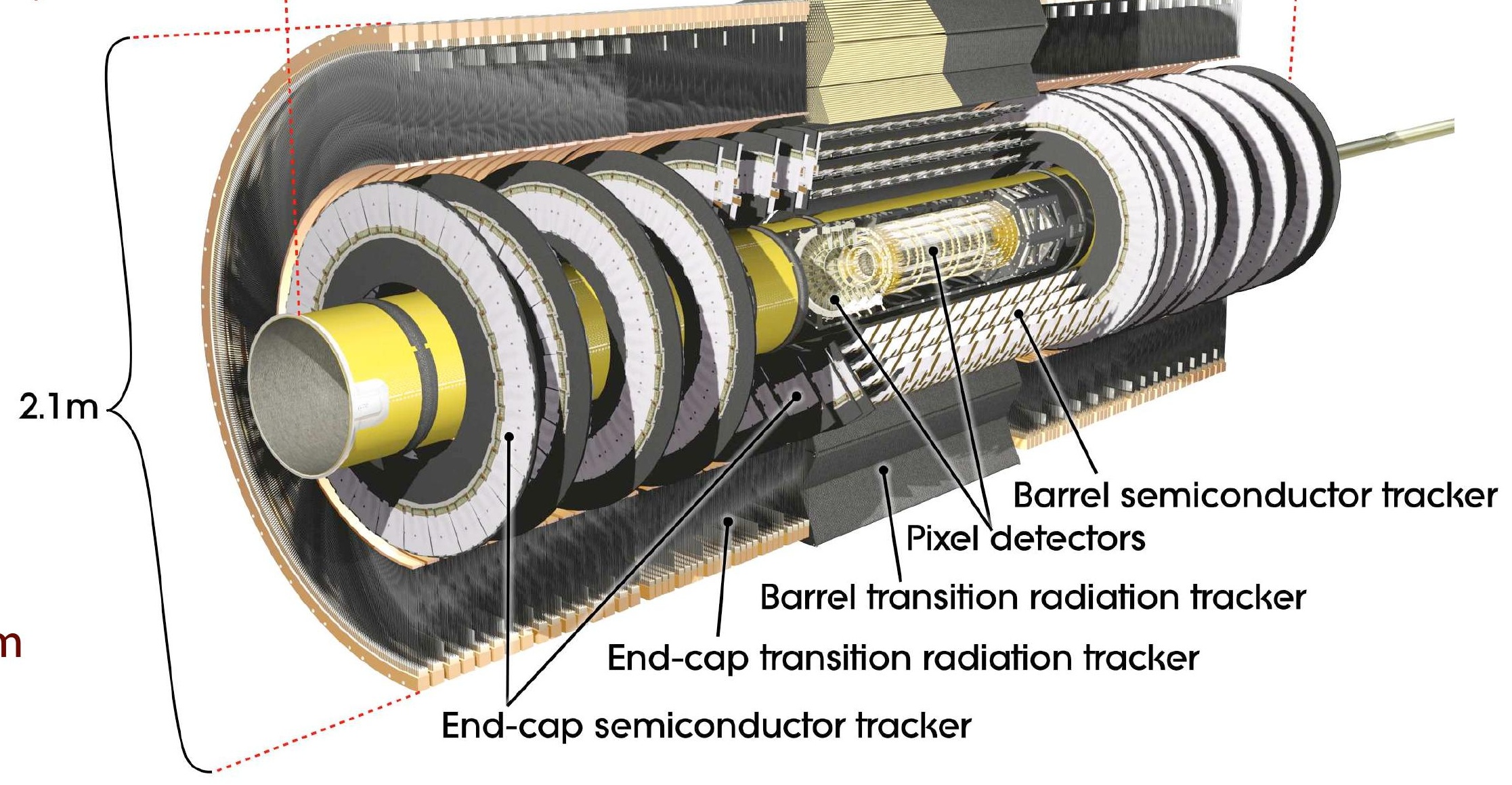




\section{Inner Detector}
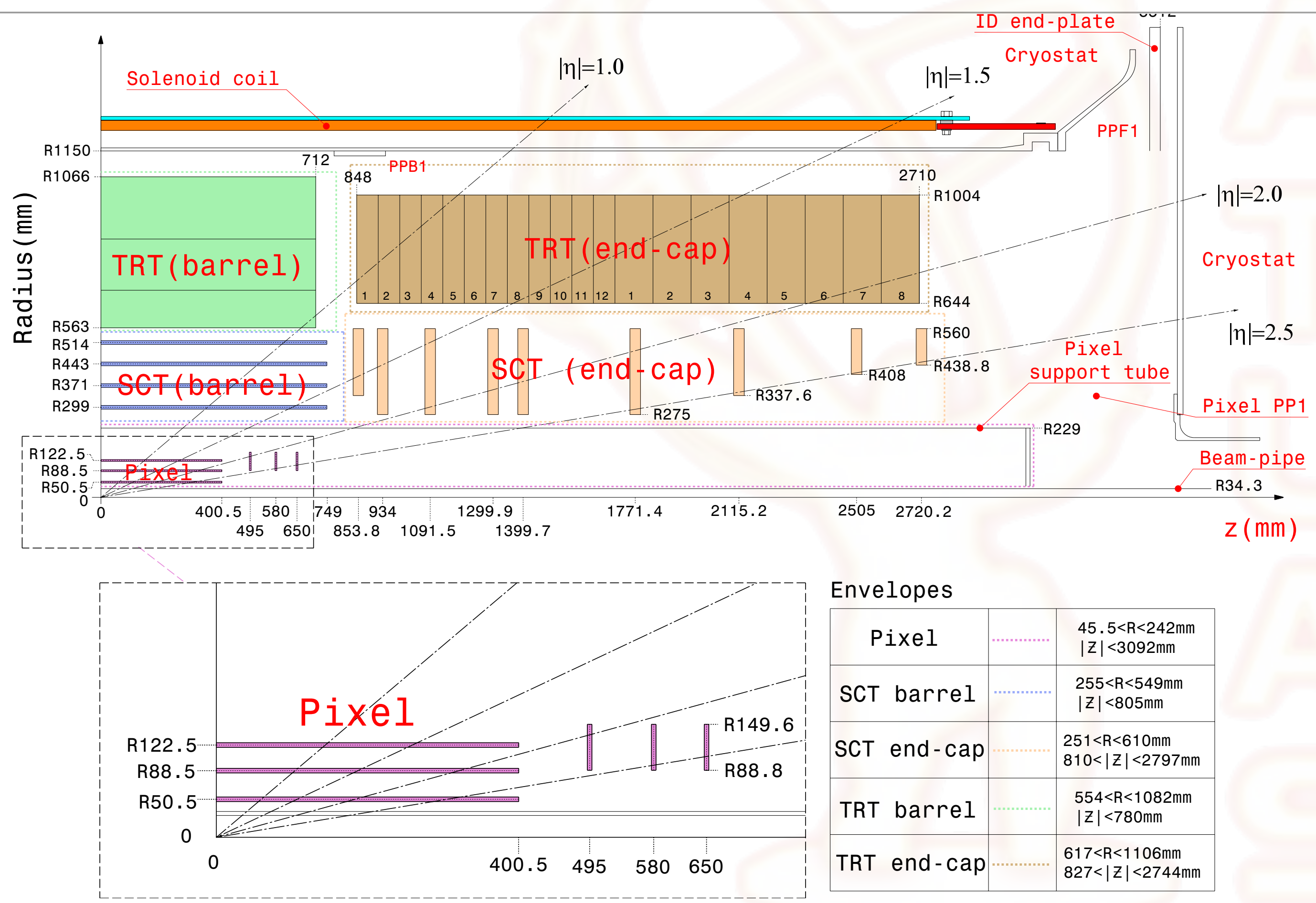

Envelopes

\begin{tabular}{|c|c|}
\hline Pixel & $\begin{array}{l}45.5<R<242 \mathrm{~mm} \\
|Z|<3092 \mathrm{~mm}\end{array}$ \\
\hline SCT barrel & $\begin{array}{l}255<R<549 \mathrm{~mm} \\
|Z|<805 \mathrm{~mm}\end{array}$ \\
\hline SCT end-cap & \begin{tabular}{|l|}
$251<R<610 \mathrm{~mm}$ \\
$810<|Z|<2797 \mathrm{~mm}$
\end{tabular} \\
\hline TRT barrel & $\begin{array}{l}554<R<1082 \mathrm{~mm} \\
|Z|<780 \mathrm{~mm}\end{array}$ \\
\hline TRT end-cap & $\begin{array}{l}617<R<1106 \mathrm{~mm} \\
827<|Z|<2744 \mathrm{~mm}\end{array}$ \\
\hline
\end{tabular}




\section{track distribution}

D performance

- Tracking

- good data/MC agreement in each detector

- e/T separation

- clearly achieved in TRT

- primary vertex

- $\sigma x, y \approx 30 \mu \mathrm{m}$

with beam spot constraint

- $\sigma z \approx 50 \mu \mathrm{m}$

- Good separation within interaction region ( $28 \mathrm{~mm}$ in the longitudinal plane)
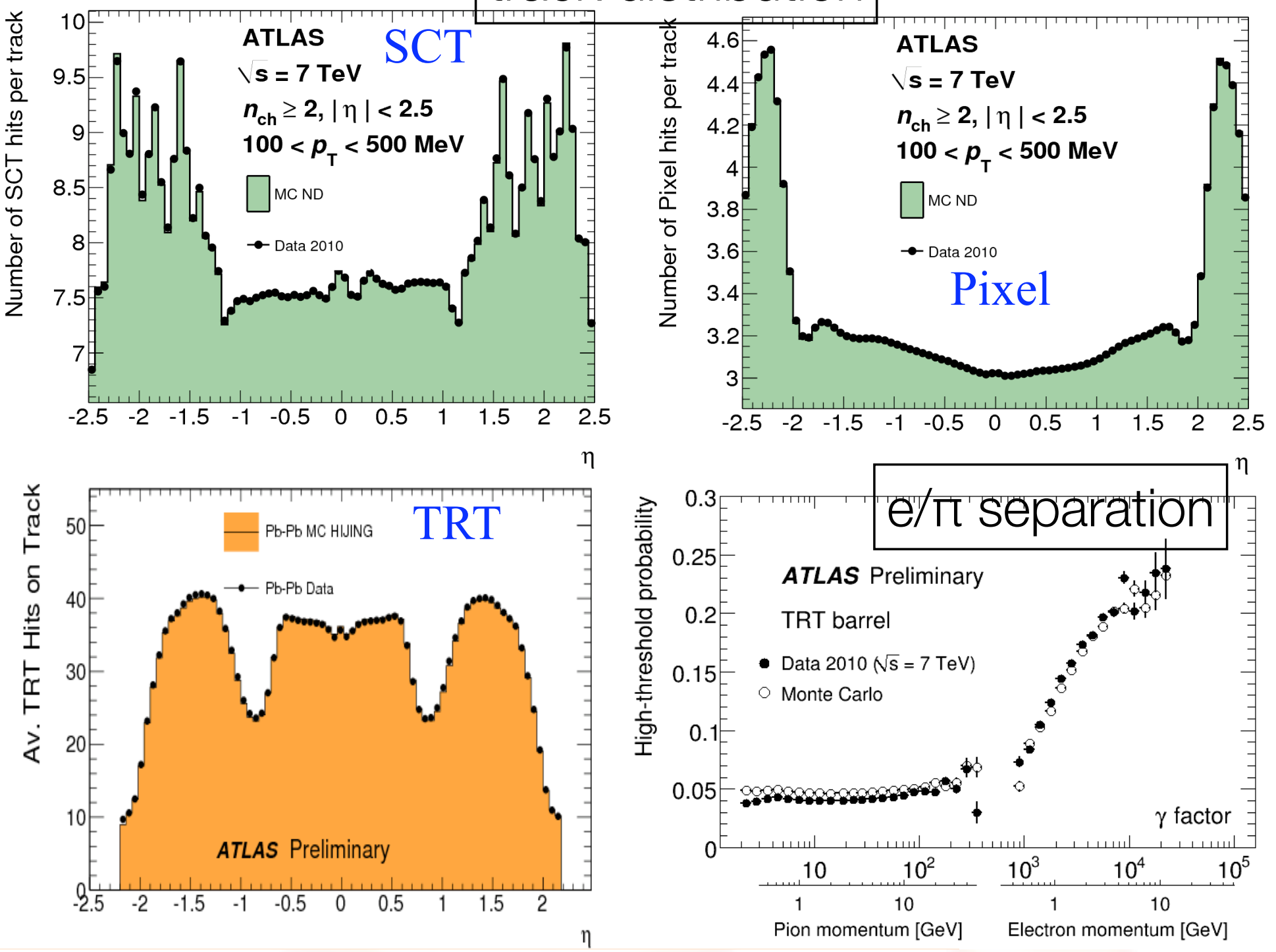

- B-tagging

- Hadrons containing b-quarks travel a few $\mathrm{mm}$ before decaying

- secondary vertices:

- tracks with large impact parameter vs primary vertex

- Main tool for top event selection and many Higgs/SUSY searches
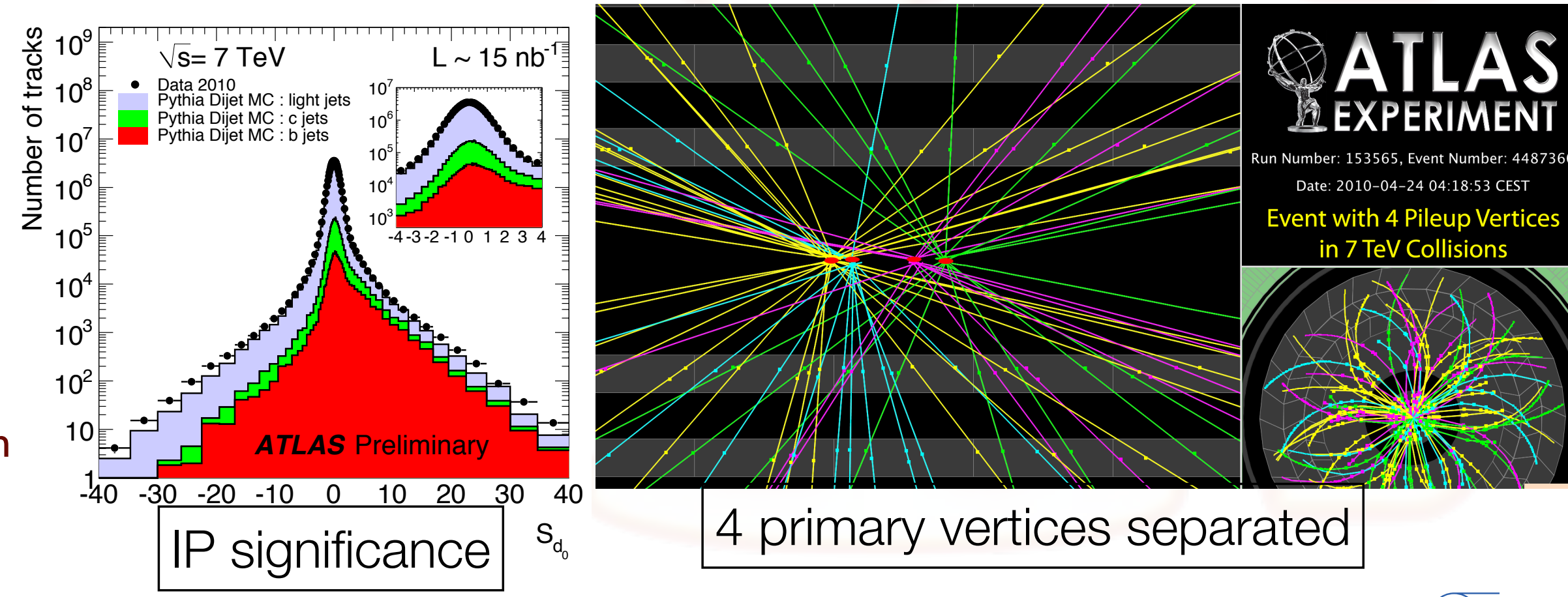


\section{Calorimeters}

- Purpose

- Energy measurement! (and if there is unbalance, i.e. ET miss)

- Liquid Argon Calorimeter (LAr):

- $\mathrm{Pb}+\mathrm{LAr}$ ionization

- coverage: $|\eta|<4.9$

- EM precise measurement in barrel

- jet measurement in endcap

- total energy measurement in the forward

- resolution

- $\sigma_{E} / E=10 \% / \sqrt{ } E \oplus 0.7 \%$

- Tile Calorimeter (Tile):

- Fe absorber + plastic scintillator tiles

- coverage: $|\eta|<1.7$

- barrel + endcap

- resolution

$$
\text { - } \sigma_{E} / E=50 \% / \sqrt{ } E \oplus 3 \%
$$

LAr electromagnetic end-cap (EMEC)

LAr hadronic end-cap (HEC)

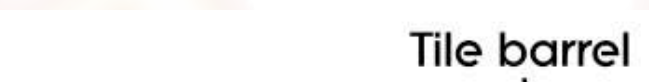

Tile barrel

Tile extended barrel

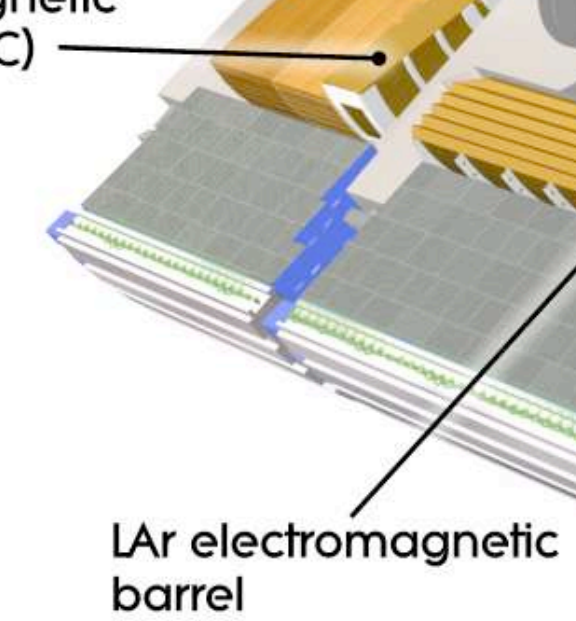

- Precise measurement of Jet energy

- goal: JES uncertainty I\% 


\section{Calorimeters}
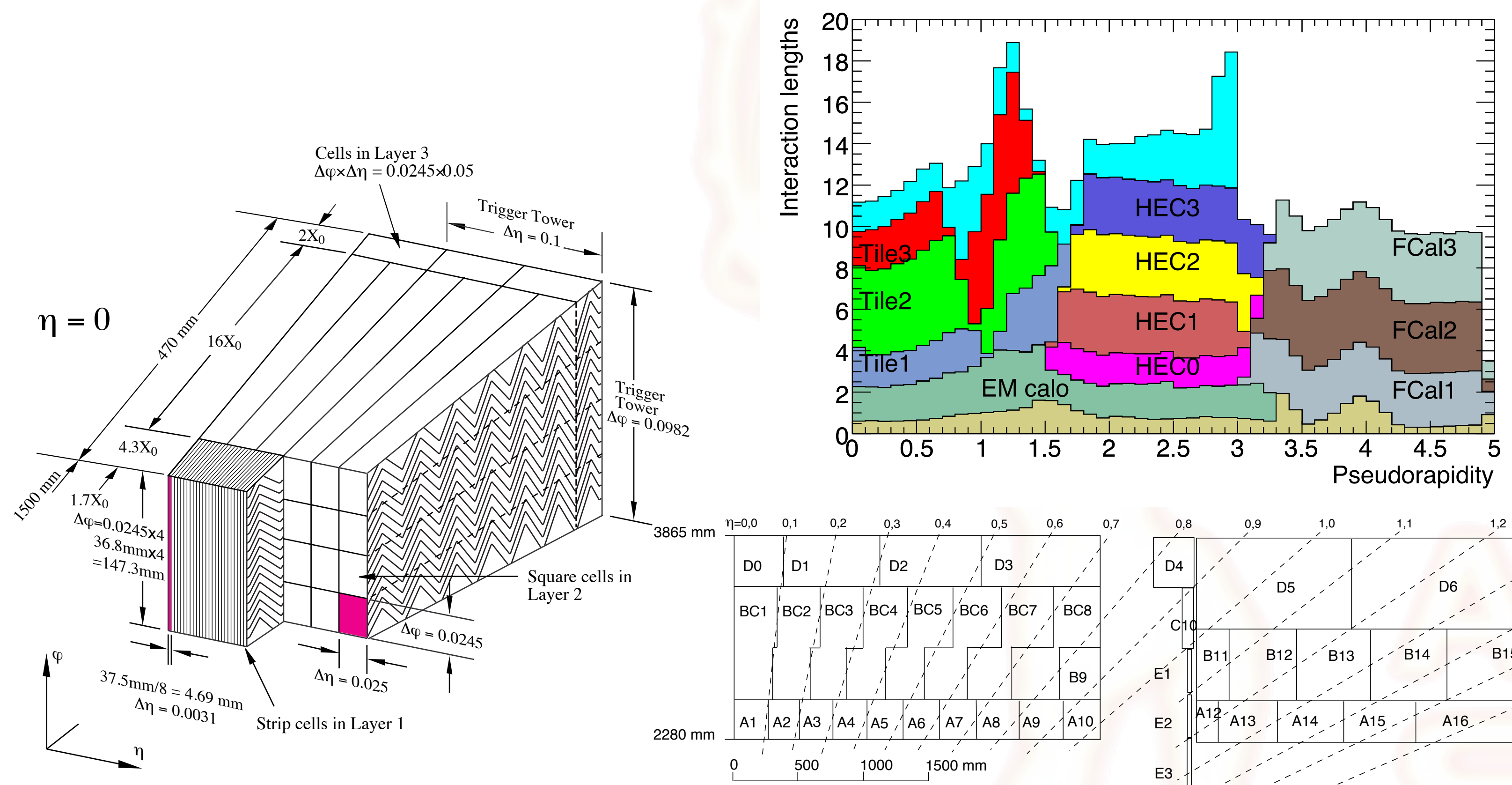

$\underset{1}{\sim}$

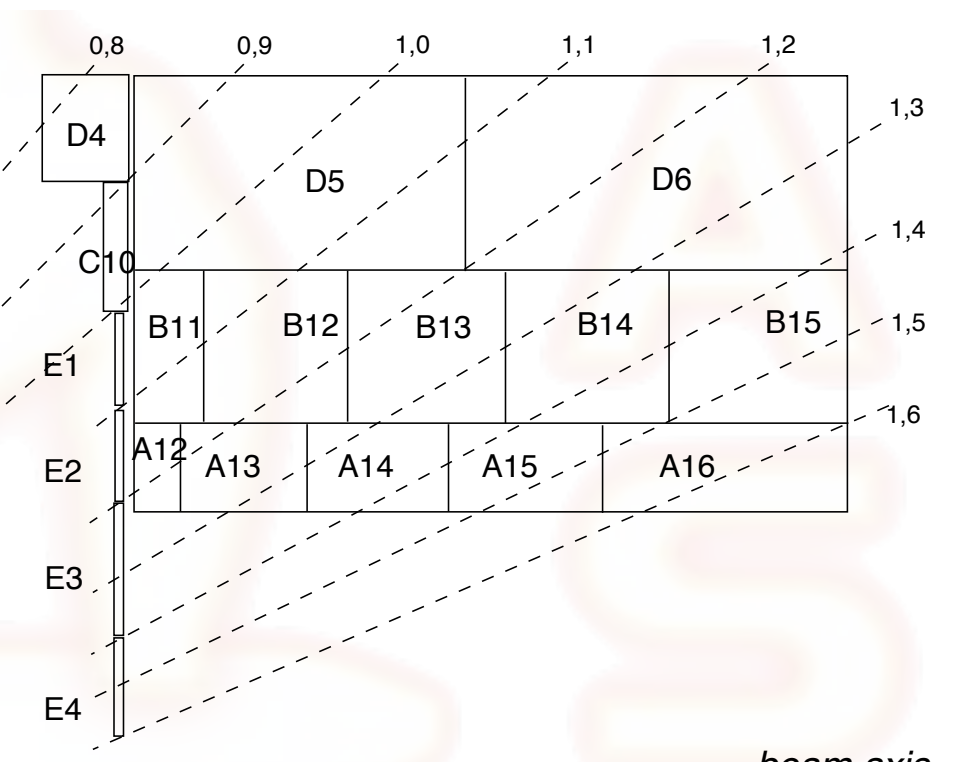

beam axis 


\section{Calo performance}

- e/r

- Purely electromagnetic clusters with right shape

- $\pi_{0}, Z^{0}$ mass

- Presence (absence) of pointing tracks

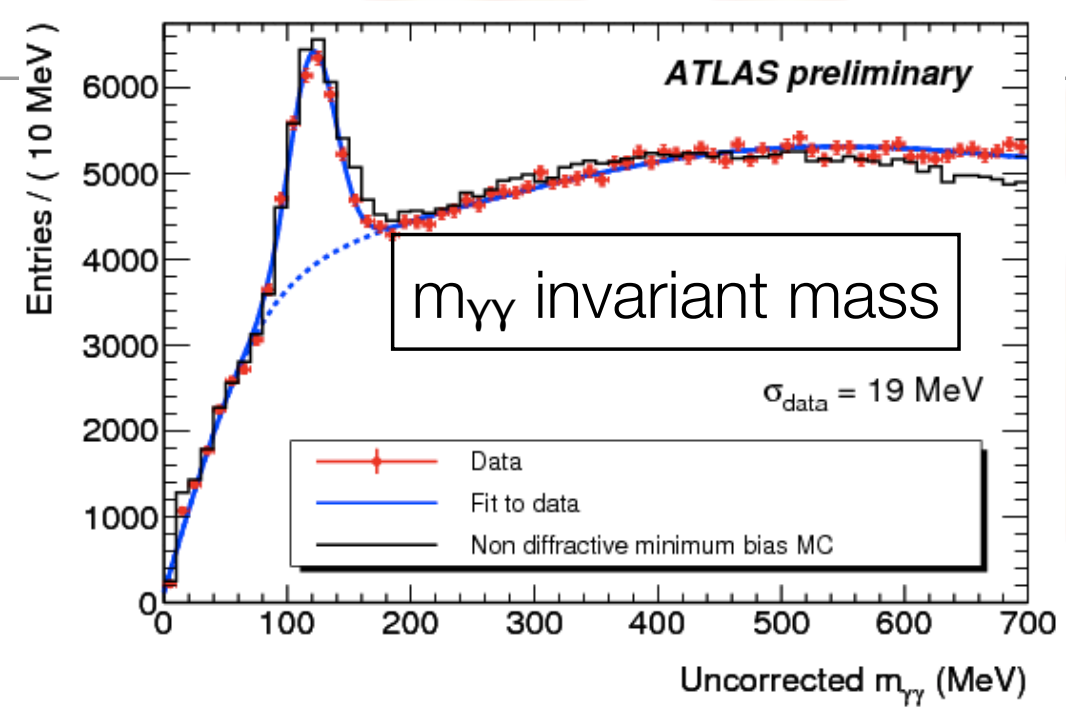

- Jet

- calibrated with MC and single charged $\pi$

- $\sigma \sim 4-5 \%$ achieved

- MET

- resolution dependence on $\Sigma \mathrm{E}_{\mathrm{T}}$

- good data/MC agreement

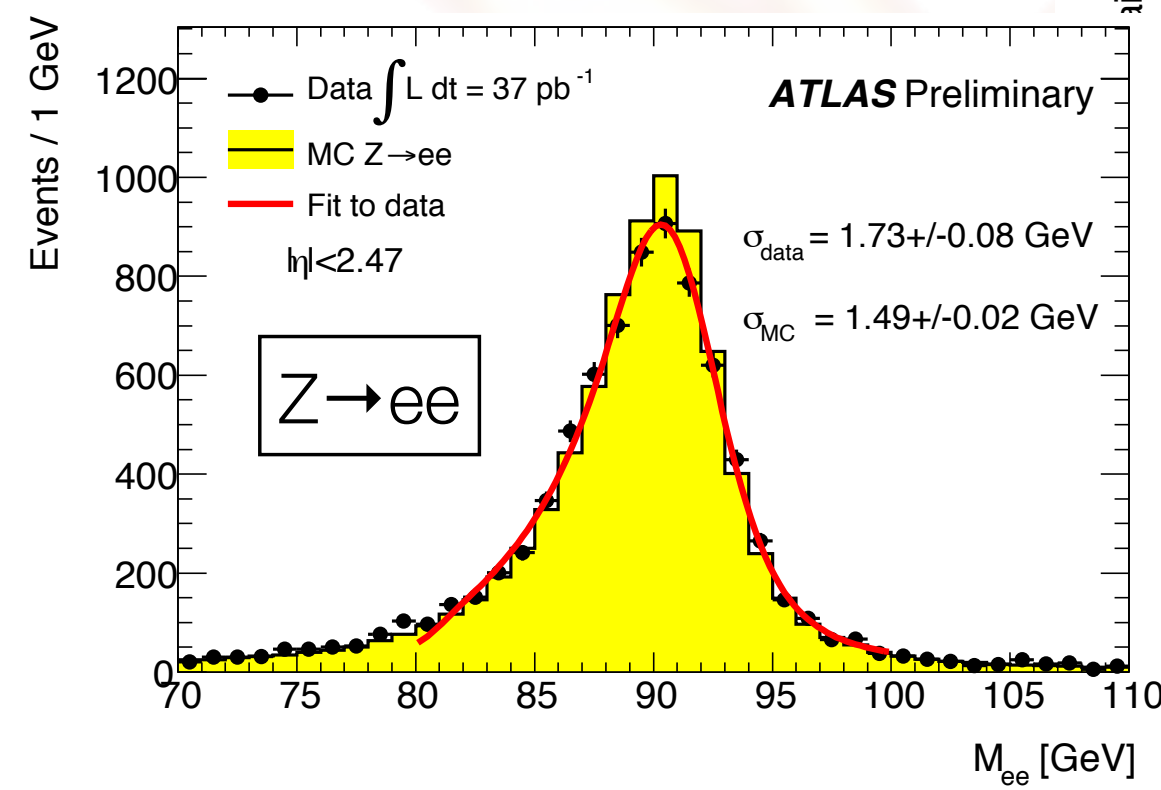

$\checkmark$ understanding of dead material in the detector
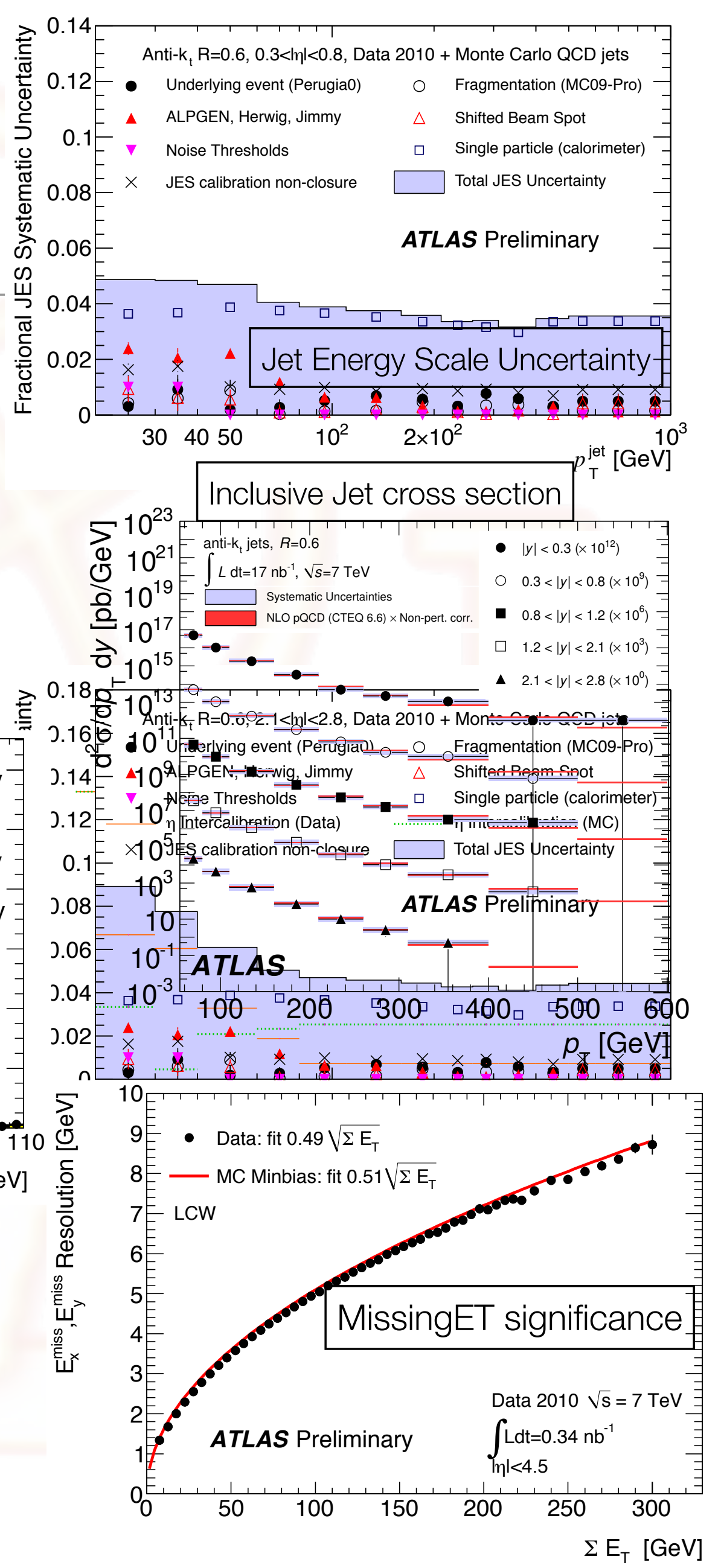


\section{Muon Spectrometers}

- Immersed in a toroidal magnetic field ( $3 \times 8$ SC toroids) in air

- $\int \mathrm{Bdl} \sim 8 \mathrm{Tm}$

- Precision detectors

- Barrel

- MDT (Monitored Drift Tubes)

- Endcap

- CDC (Cathode Drift Chambers)

- Trigger chambers

- Barrel

- RPC (Resistive Plate Ch.)

- Endcap

- TGC (Thin-Gap Ch.)

- coverage: $|\eta|<2.7$

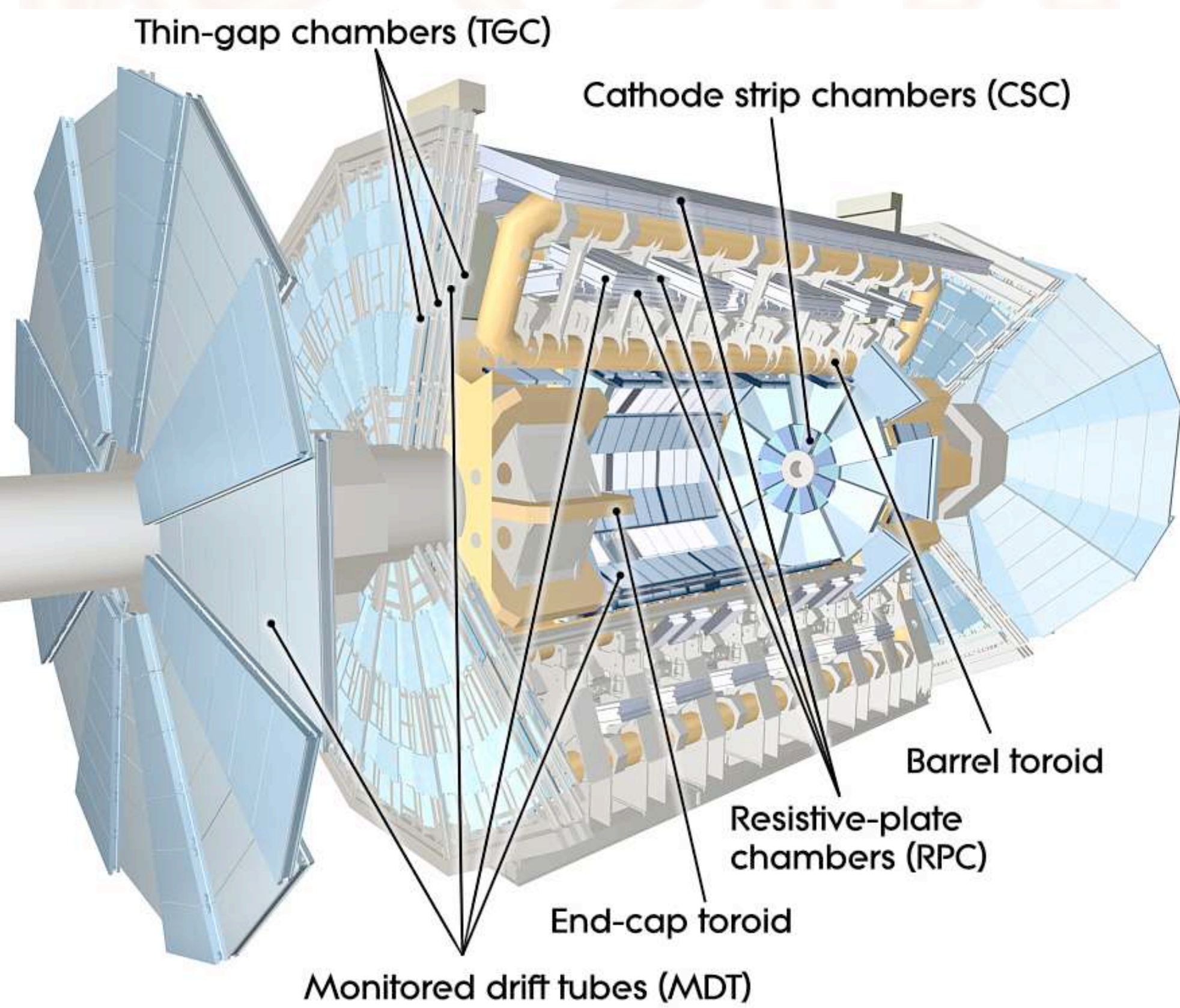




\section{Muon Spectrometers}
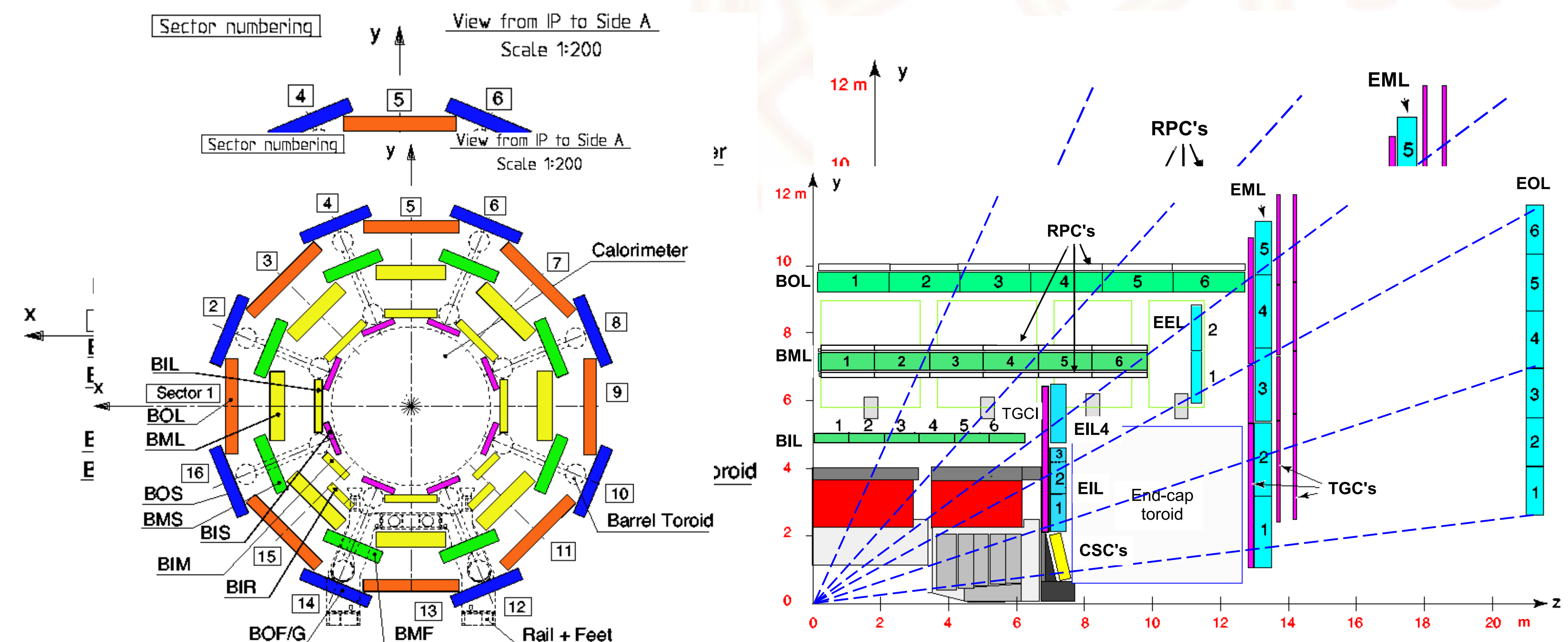


\section{Combined muon performance}

- Di-muon resonance

- All known resonances found again

- detector calibration resolution understood very well

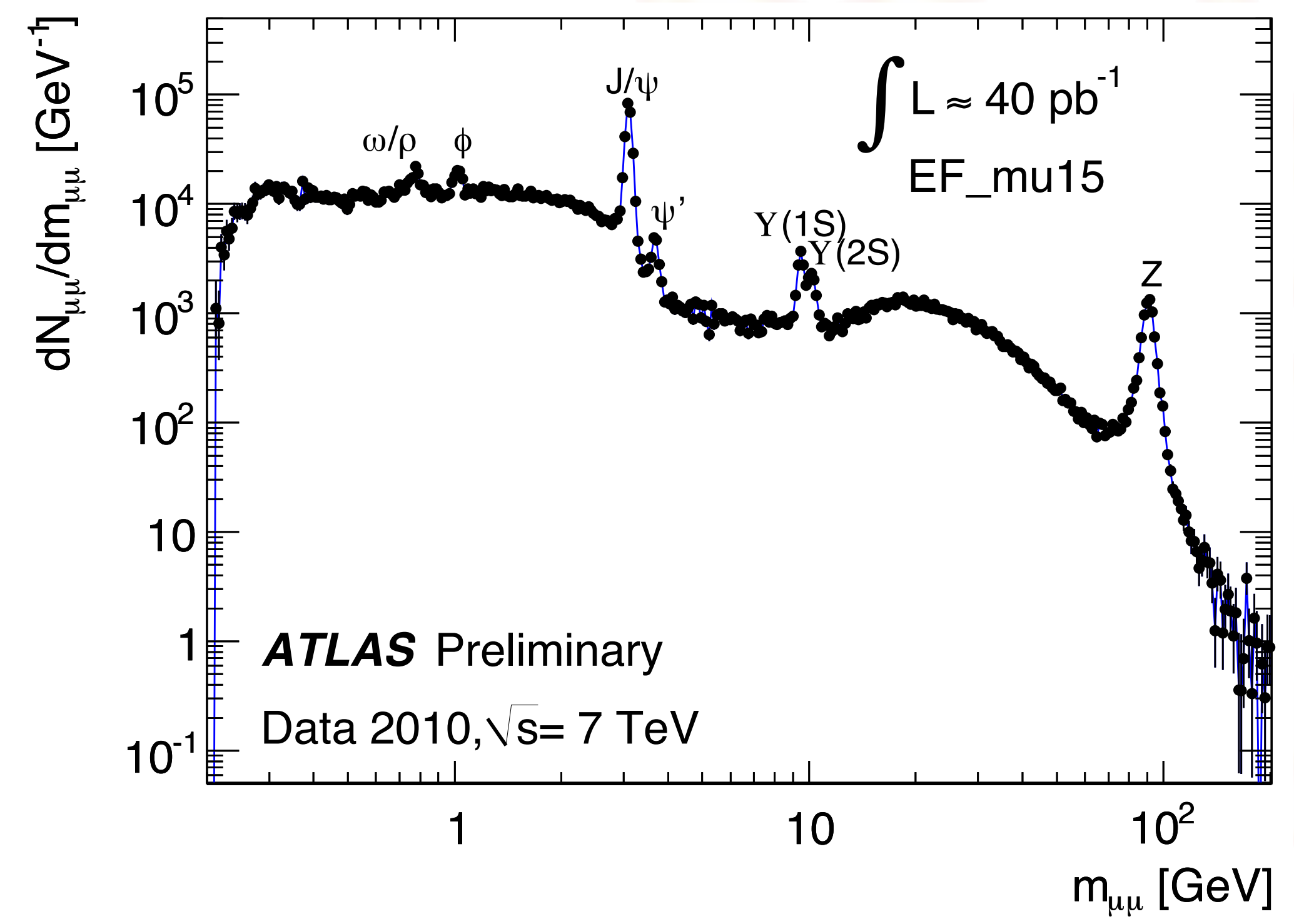




\section{Trigger}

- Online event selection

- Level-I : Hardware

- mainly muon spectrometers and calorimeters

- coarse granularity

- $\mathrm{e} / \mathrm{\gamma}, \mu, \pi, \mathrm{T}$, jet candidate selection

- Define regions of interest (ROls)

- Level-2 : Software

- Seeded by level-I ROls, full granularity

- Inner Detector - Calo track matching

- Event Filter: Software

- Offline-like algorithms for physics signatures

- Refine LV2 decision

- Full event building

- Rate ( 2010 / nominal(design) )

- Bunch crossing: I MHz / $40 \mathrm{MHz}$

- Level- I: $20 \mathrm{kHz} / 75 \mathrm{kHz}$

- Level-2: $3.5 \mathrm{kHz} / \mathrm{l} \mathrm{kHz}$

- EF: $300 \mathrm{~Hz} / 100 \mathrm{~Hz}$

- Challenges

- Coping very well with rapidly increasing luminosity by adjusting pre-scales/menus

- need to be optimize sharing of the bandwidth for physics

- final event rate by EF will be doubled in 20I I, skimming the data size
Interaction rate
$\sim 1 \mathrm{GHz}$

Bunch crossing rate $40 \mathrm{MHz}$

LEVEL 1

$<75$ (100) $\mathrm{kHz}$

Regions of Interest

LEVEL 2 IRIGGER

$\sim 1 \mathrm{kHz}$
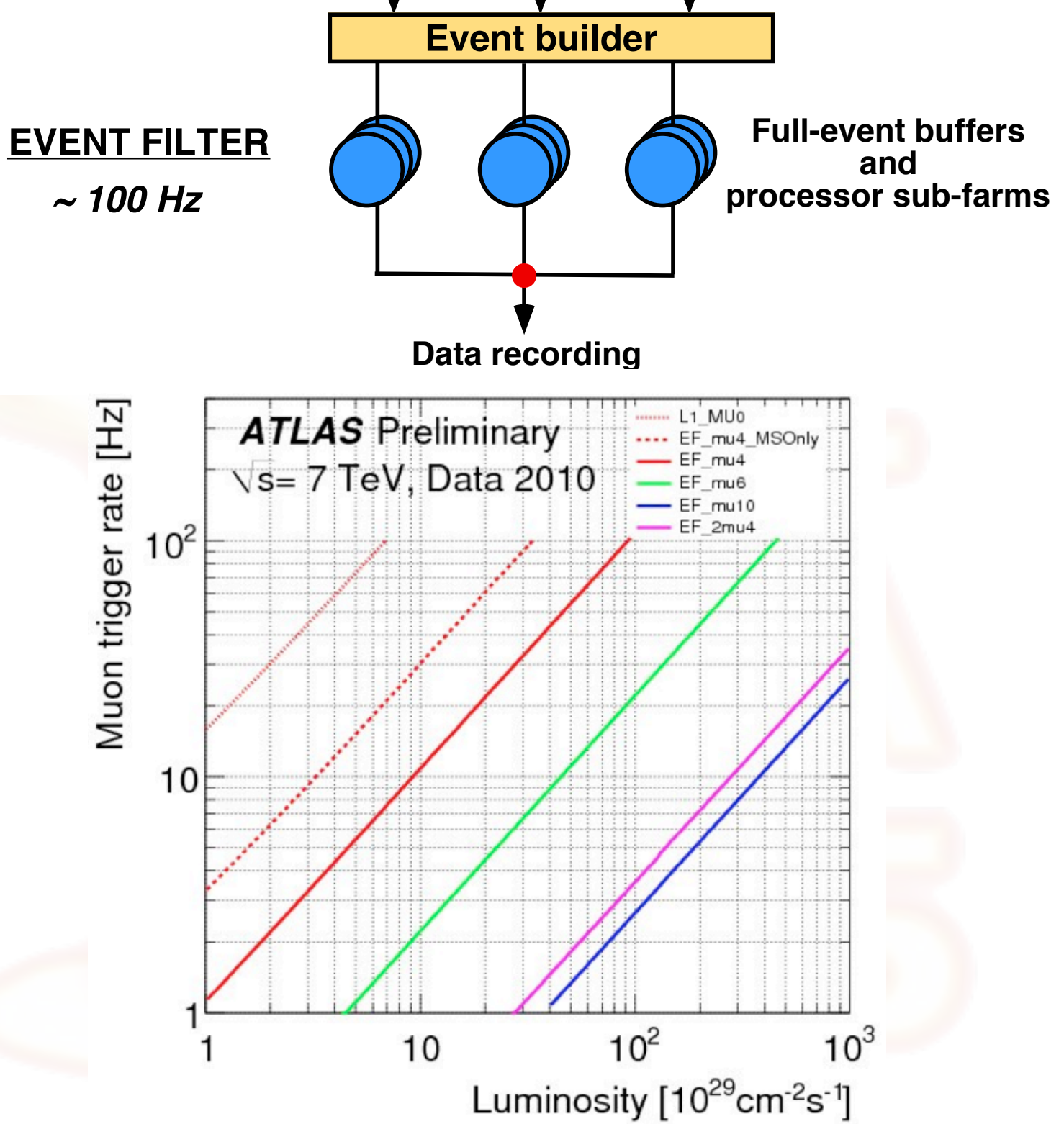


\section{ATLAS detector performance}

- Very stable performance despite dramatic increase in luminosity

- Fraction of operational channels

- $90-100 \%$ depending by sub-detector

- $75-85 \%$ of delivered luminosity used for analysis with first-pass processing

- improving in reprocessing

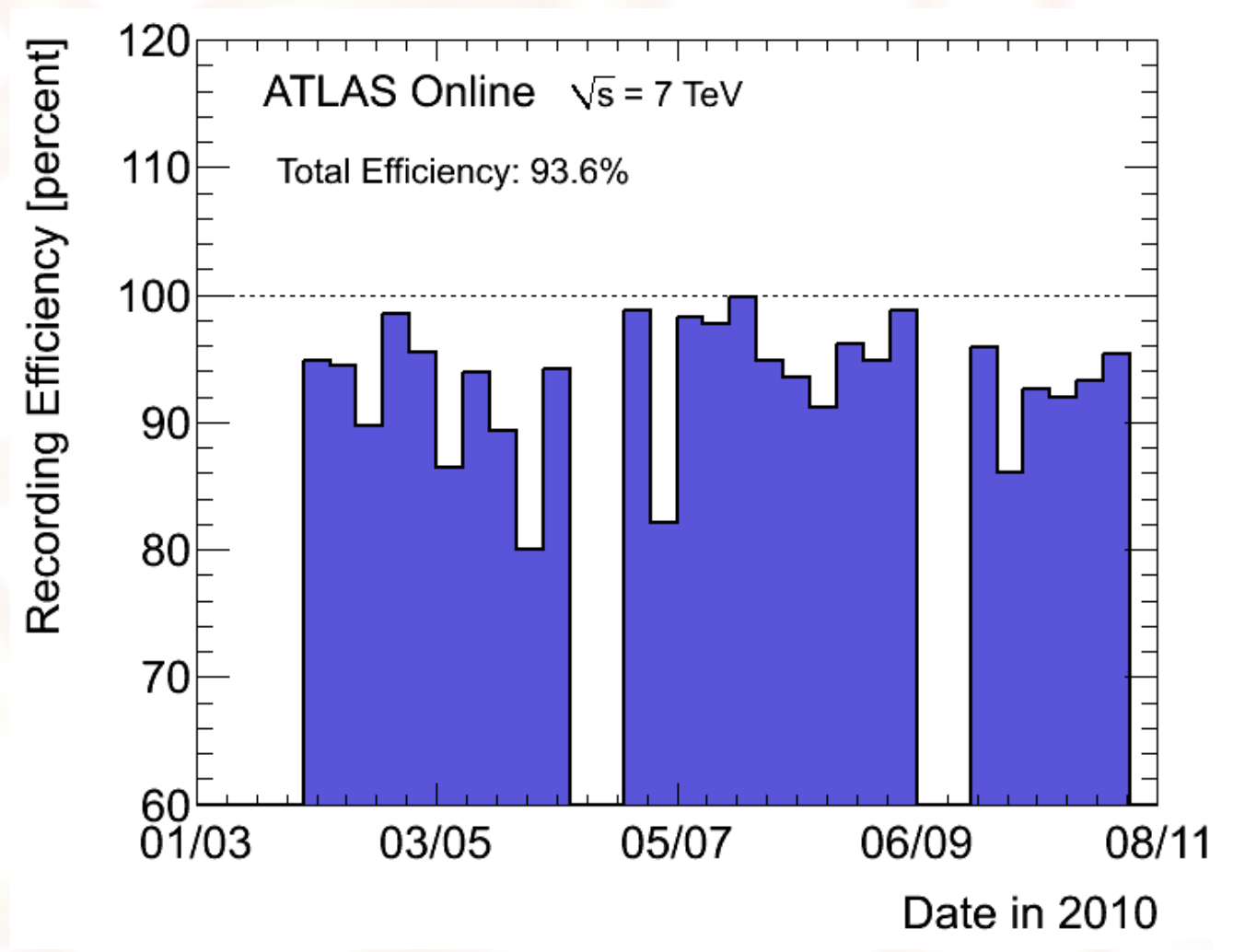

\begin{tabular}{|c|c|c|c|c|c|c|c|c|c|c|}
\hline \multicolumn{3}{|c|}{ InnerDetector } & \multicolumn{4}{c|}{ Calorimeters } & \multicolumn{4}{c|}{ Muonspectrometer } \\
\hline Pixel & SCT & TRT & $\begin{array}{c}\text { LAr } \\
\text { EM }\end{array}$ & $\begin{array}{c}\text { LAr } \\
\text { HAD }\end{array}$ & $\begin{array}{c}\text { LAr } \\
\text { FWD }\end{array}$ & Tile & MDT & CSC & RPC & TGC \\
\hline 99.0 & 99.9 & 100 & 90.7 & 96.6 & 97.8 & 94.3 & 99.9 & 96.2 & 99.8 & 99.8 \\
\hline
\end{tabular}

- Luminosity weighted relative percentage of good quality data delivery by the various ATLAS subsystems during LHC fills with stable beams in PP collisions at $\sqrt{ } s=7 \mathrm{TeV}$, and after switching the tracking detectors on. 


\section{Magnet splices and beam energy}
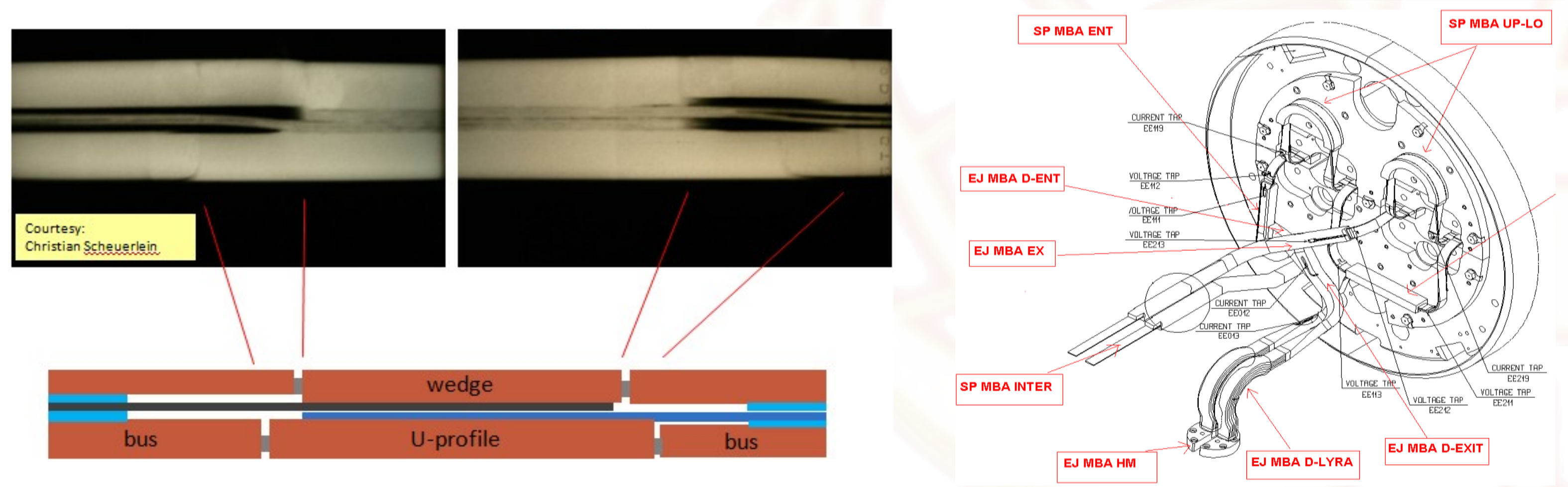

- Missing continuity in the copper stabilizer and contact with the superconducting (SC) cable effectively reduces safe current level

- In case of quench the cable locally acts like a "fuse" and burns out

- Value of safe current depends on additional resistance of worst splice

- Not all splices measured so far: it is then considered unsafe to run at more than $3.5 \mathrm{TeV}$

- Direct measurement method to be deployed by 20 II winter stop

- "Thermal Amplifier": measure of thermal runaway of a bad joint under a pulse of high current (order 3000A)

- According to the results of the measurement campaign we may decide to increase beam energy in 2012 


\section{Jet Vertex Fraction}

- Associate jets to primary vertices using tracks and obtain a jet-selection criterion and jetby-jet energy correction

- Tracks are selected with standard quality criteria and required to be matched to a vertex - not required to be unique ( $2 \mathrm{~mm}$ window)

- JVF measures the fraction of charged particle transverse momentum in each jet originating in each identified primary vertex in the event.

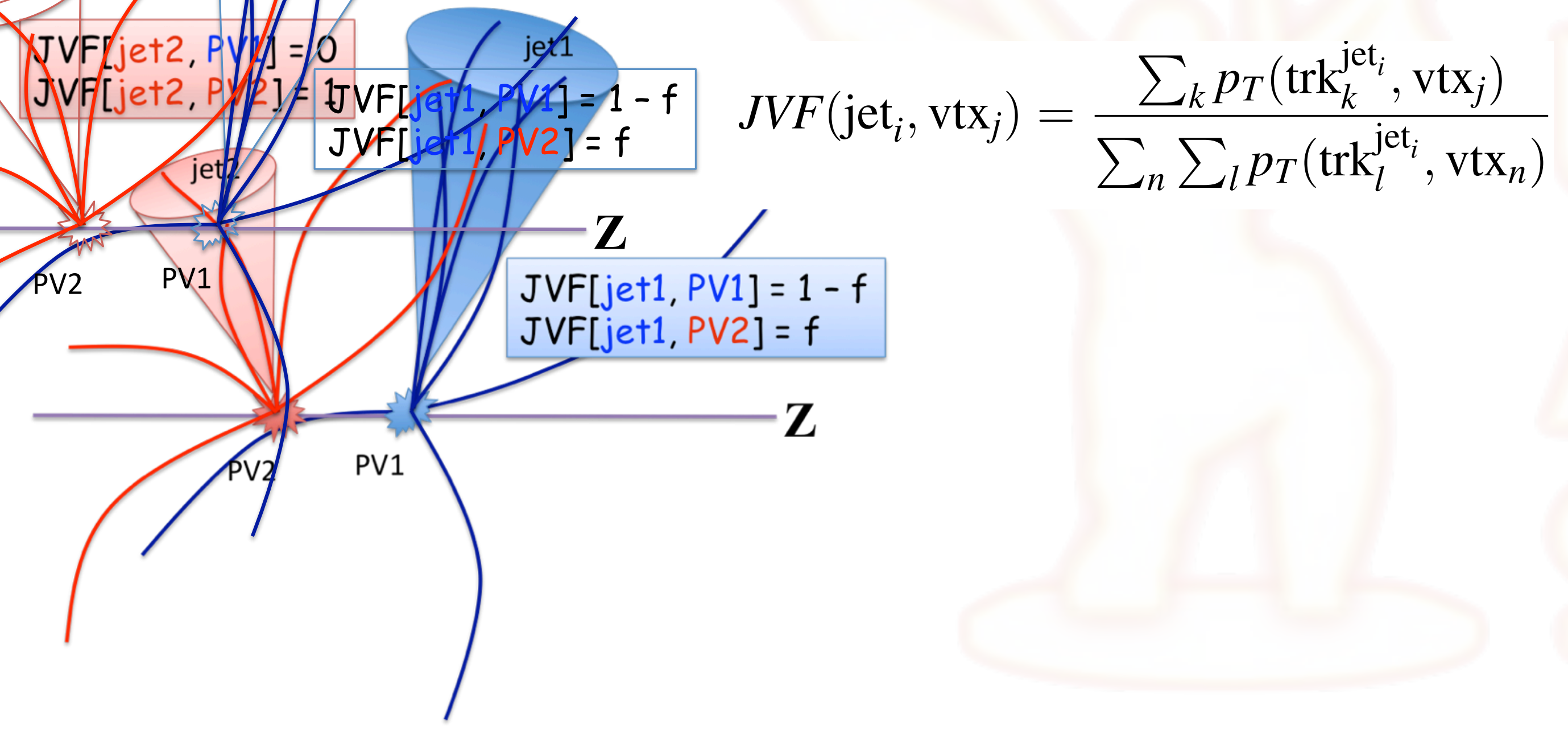




\section{B-tagging}

- Algorithms

- SVO

- lifetime tagger

- reconstruct secondary vertex

- cut on decay length significance - JetProb

- lifetime tagger

- compare impact parameter significance to resolution function

- gives probability track originates from primary vertex

- all tracks in a jet combined, giving $P$ of no long-lived particles
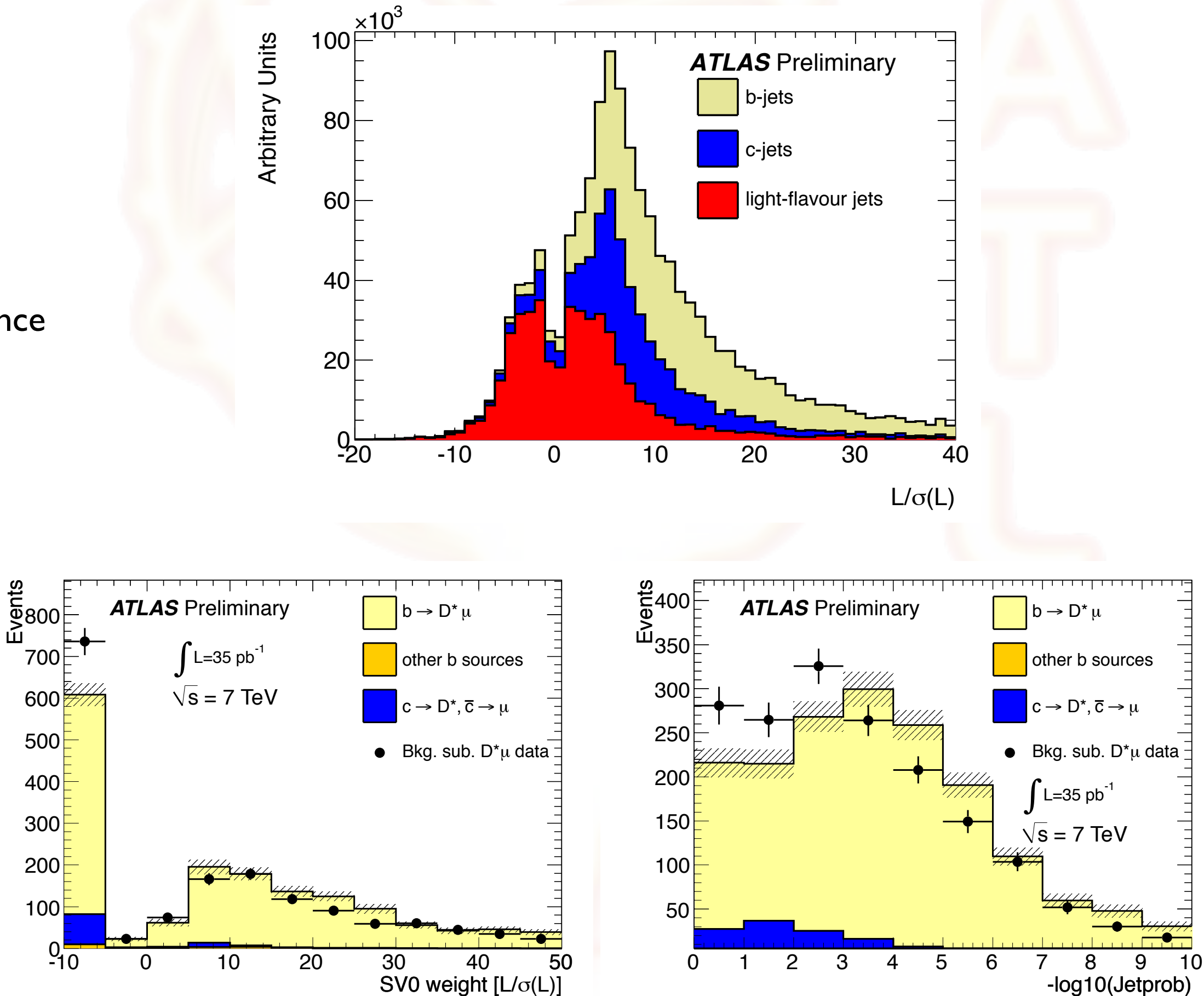

ATLAS-CONF-2011-089 
Complementary results

S




\section{Inclusive jet cross section}

- Double-differential cross section

- systematic uncertainty

- JES

- unfolding

- luminosity

- total: $20 \%$

- Result in 2010 data $\left(37 \mathrm{pb}^{-1}\right)$

- relatively good agreement in central region

- larger discrepancy

in forward region
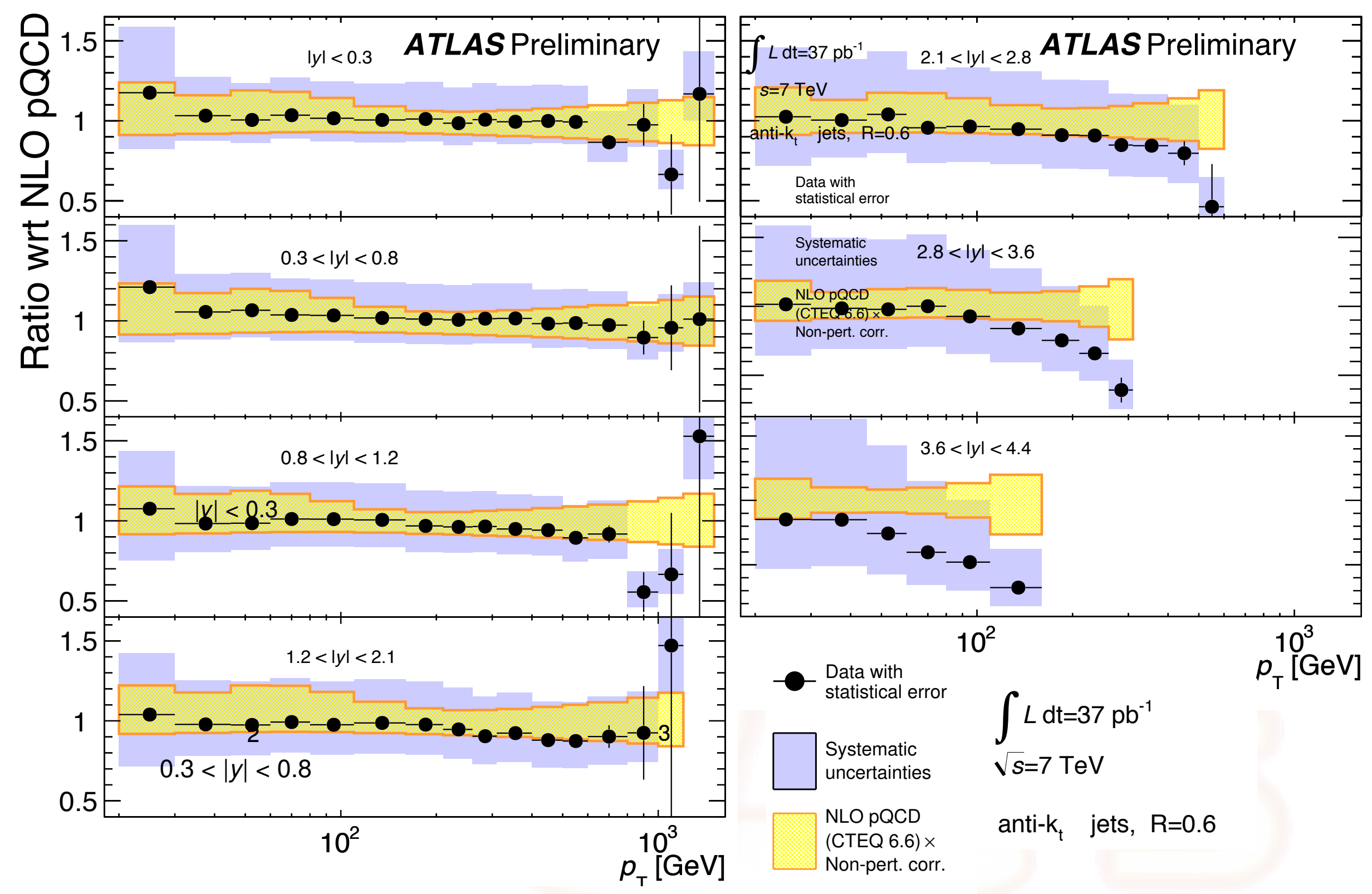

ATLAS-CONF-2011-047 


\section{B-jet production}

- Selection

- $\mathrm{PT}_{\mathrm{T}}>20 \mathrm{GeV},|\mathrm{y}|<2$. I

- secondary vertex b-tagging

- Uncertainties

- JES + b-jet energy scale: $35-40 \%$

- b-tagging efficiency: 10-20\%

- Result in $3 \mathrm{pb}^{-1}$ (full dataset in preparation)

- predictions in agreement with data, but slightly softer

- Pythia (LO*)

- POWHEG (NLO)

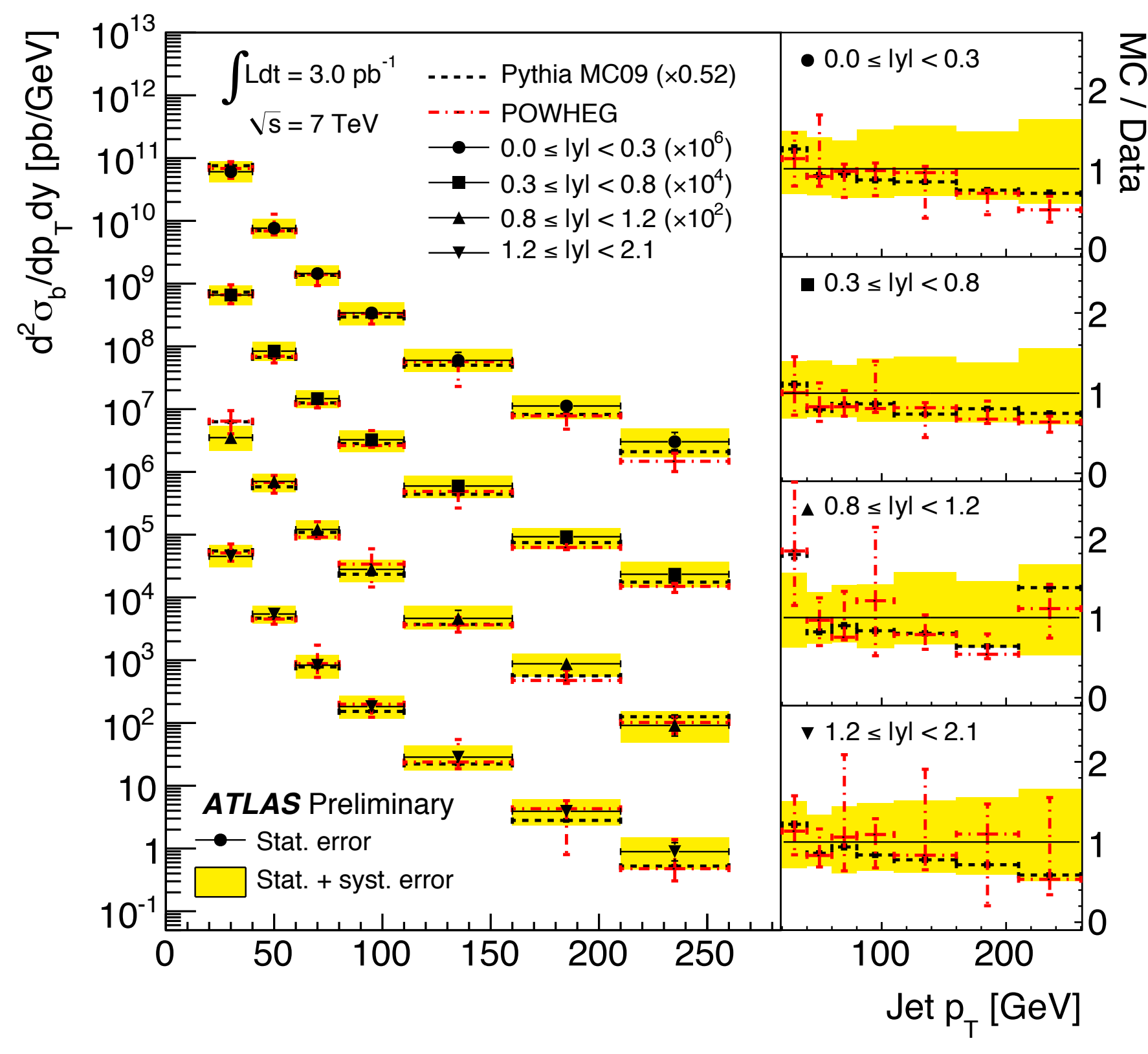

ATLAS-CONF-2011-056 


\section{Dijet production with a jet veto}

- Events with no additional jets

in rapidity interval spanned by dijet system

$\checkmark$ gap fraction

- test PQCD radiation in dijet system for large $\Delta y$, high PT

- test wide angle soft gluon radiation (BFKL dynamics)

- jet vetos in vector boson fusion analyses (Higgs +2 jets)

- Event selection

- exactly I primary vertex

- dijet system from 2 leading jets

- $\overline{\mathrm{PT}}>50 \mathrm{GeV},|\mathrm{y}|<4.5$

$$
\checkmark \overline{\mathrm{P} T}=\left(\mathrm{PT}^{1}+\mathrm{PT}^{2}\right) / 2
$$

- Systematic uncertainties

- absolute and relative JES between jets

- Comparisons with several generators

- Pythia 6 gives good description

- HERWIG++ and ALPGEN overestimate gap radiation

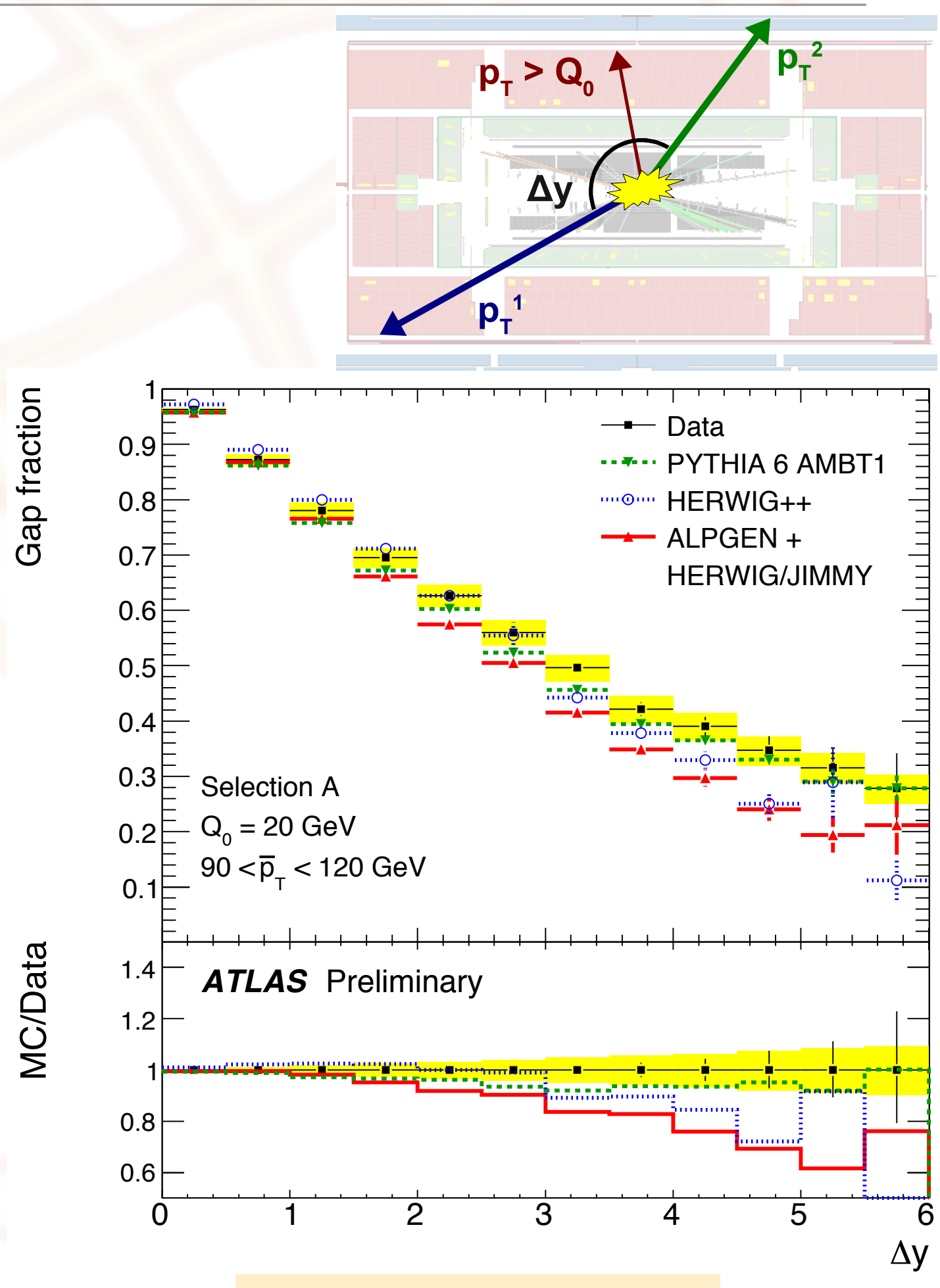

ATLAS-CONF-2011-038 


\section{Jet mass and substructure}

- Mass and substructure in large jets ( $R=I .0$ and $I .2$ are studied)

- To identify boosted objects which can decay hadronically inside the jet

- electroweak objects, Higgs

- top

- To define splitting scale

- $\sqrt{ } d_{12}=\min (\mathrm{PTa}, \mathrm{PTb}) \times \delta R_{\mathrm{a}, \mathrm{b}}$

- Systematics

- JES and jet energy resolution (JER) for larger size jets

- unfolding

- Result for jets with $R=1.0\left(35 \mathrm{pb}^{-1}\right.$ in 2010)

- good agreement between data and MC
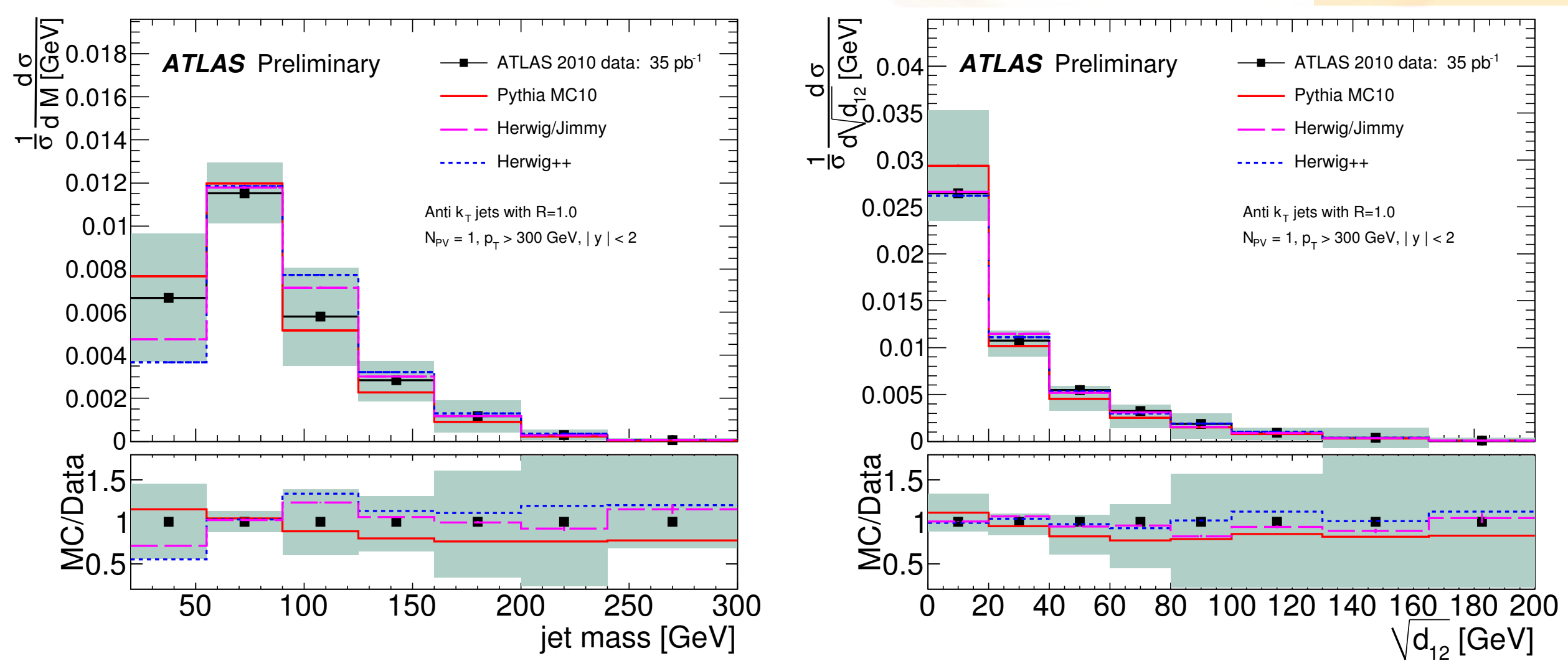


\section{W charge asymmetry}

- Definition of lepton charge asymmetry from $\mathrm{W}$ bosons

- $A_{\mu}=\frac{d \sigma_{W_{\mu^{+}}} / d \eta_{\mu}-d \sigma_{W_{\mu^{-}}} / d \eta_{\mu}}{d \sigma_{W_{\mu^{+}}} / d \eta_{\mu}+d \sigma{W_{\mu^{-}}} / d \eta_{\mu}}$

- dominant production process

- $\mathrm{u} \overline{\mathrm{d}}(\mathrm{u} d) \rightarrow \mathrm{W}^{ \pm}$

- if more valence u-quark in the proton

$\checkmark \mathrm{W}^{+}$is favored over $\mathrm{W}^{-}$

- Signal selection

- pT $\mu>20 \mathrm{GeV}$

- asymmetry as a function of $\eta$

- Dominating systematic uncertainties

- reconstruction efficiency

- muon trigger efficiency

- theoretical modeling
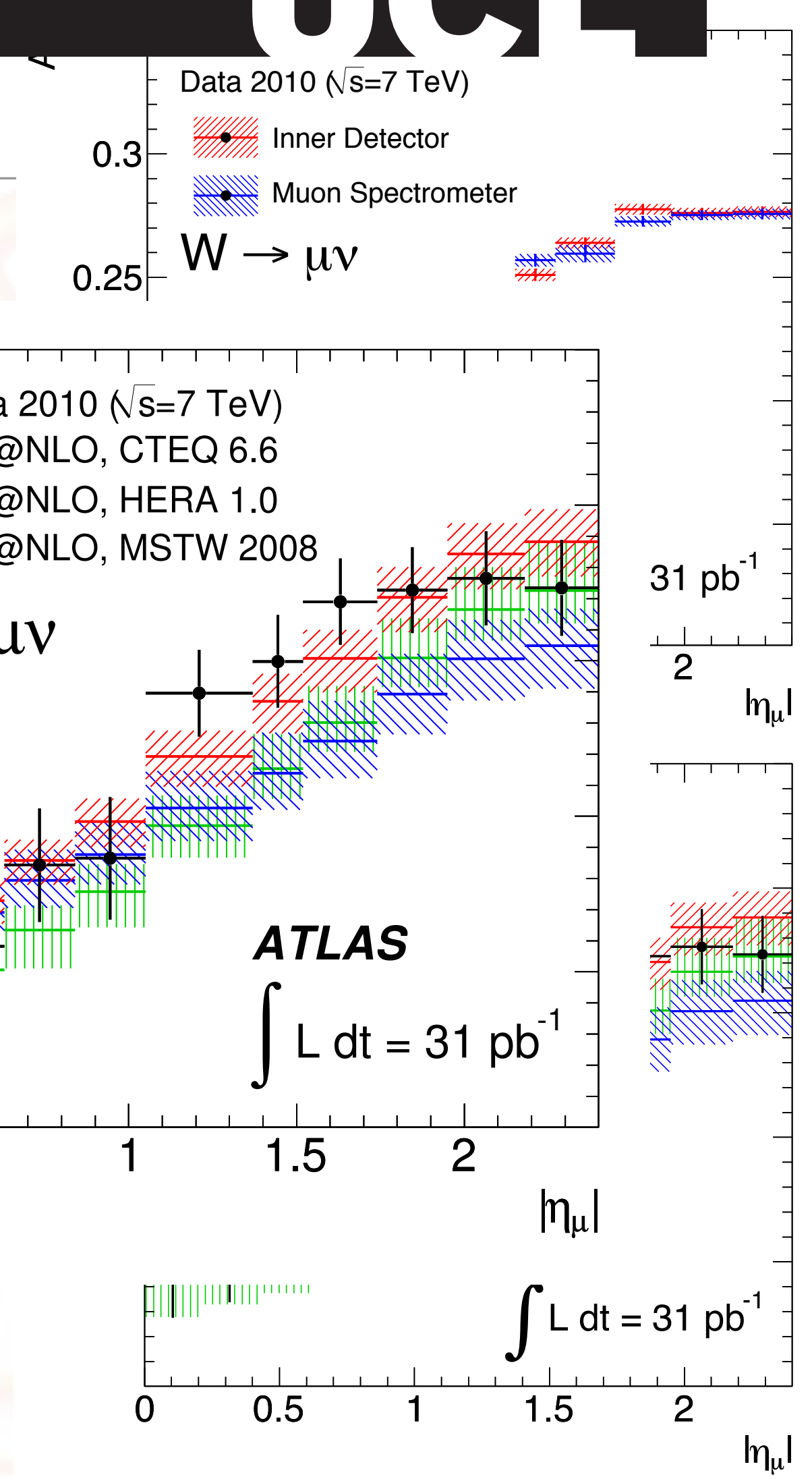

arXiv: 1103.2929, accepted by PLB 


\section{$Z \rightarrow \mathrm{TT}$}
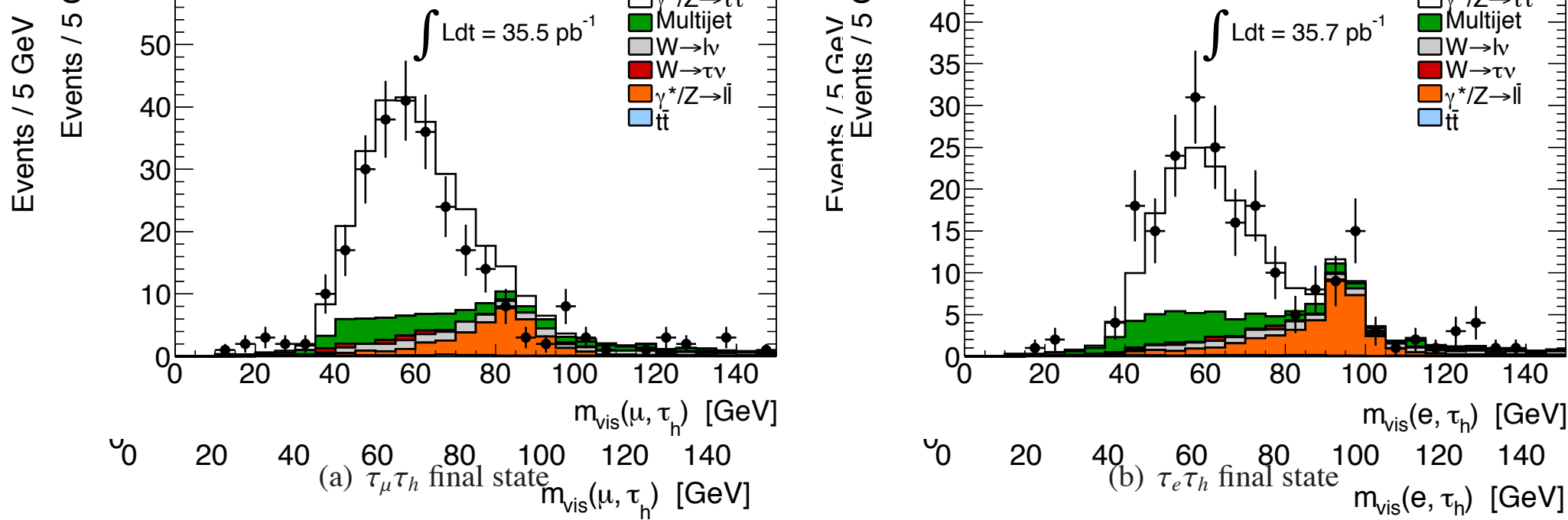

- Measured using four channels

- $Z \rightarrow T_{\mu} T_{h}$

- $Z \rightarrow T_{\mathrm{e}} T_{h}$

- $Z \rightarrow T_{\mathrm{e}} \mathrm{T}_{\mu}$

- $Z \rightarrow T_{\mu} T_{\mu}$

- Main backgrounds

- Z $\rightarrow \|$

- multi-jet events
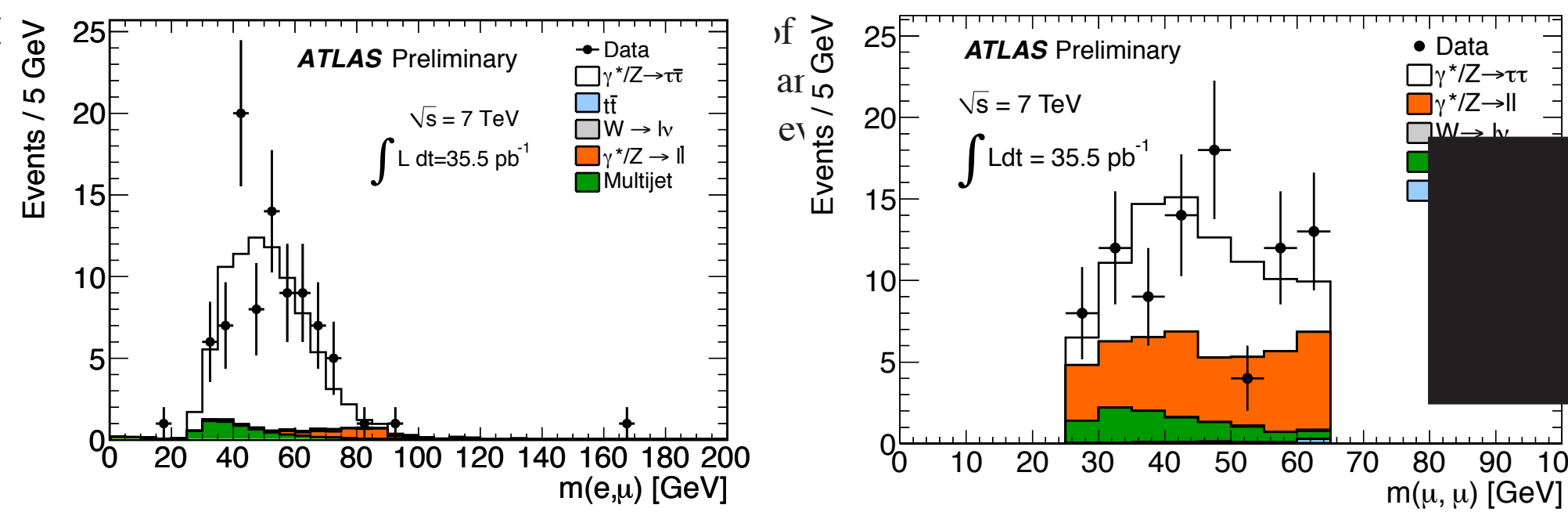

- Results

- T reconstruction is performing well

- important step towards $\mathrm{H} \rightarrow \mathrm{TT}$

- combined result

- $\sigma_{\mathrm{Z}} * \mathrm{Br}(\mathrm{Z} \rightarrow \mathrm{TT})$

$=0.97 \pm 0.07$ (stat.) \pm 0.07 (syst.) \pm 0.03 (lumi.) nb

- prediction: $0.96 \pm 0.05 \mathrm{nb}$

$\checkmark$ good agreement with SM expectations

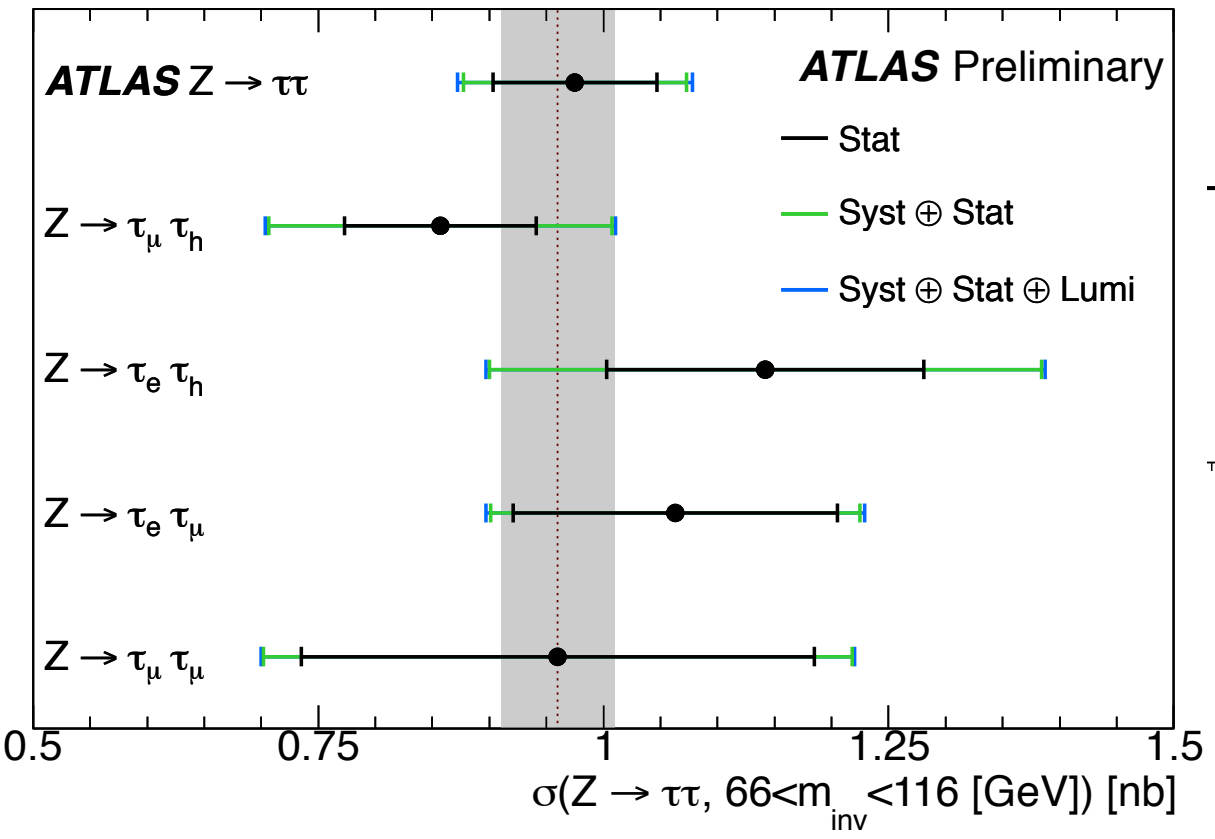

ATLAS-CONF-2011-045 + approved plots 


\section{W polarization in top decays}

- Probe V-A structure of Wtb vertex

- SM expectation for polarization fractions

- left-handed $\mathrm{F}_{\mathrm{L}} \approx 0.3$

- longitudinal $F_{0} \approx 0.7$

- right-handed $\mathrm{F}_{\mathrm{R}} \approx 0$

- observable " $\cos \theta *$ "

- decay angle of charged lepton w.r.t. top boost direction in $\mathrm{W}$ rest frame

- Two analysis techniques

- fit left-handed, longitudinal, right-handed templates to measured $\cos \theta^{*}$

- extract asymmetries (e.g. $\left.A^{F B}\right)$ from $\cos \theta^{*}$

- correct back to parton level

- Results

- $F_{0}=0.59 \pm 0.12$

- $F_{L}=0.4 I \pm 0.12$

- $F_{R}$ fixed to 0

- Interpretation

- limits on anomalous couplings $g L, g_{R}$
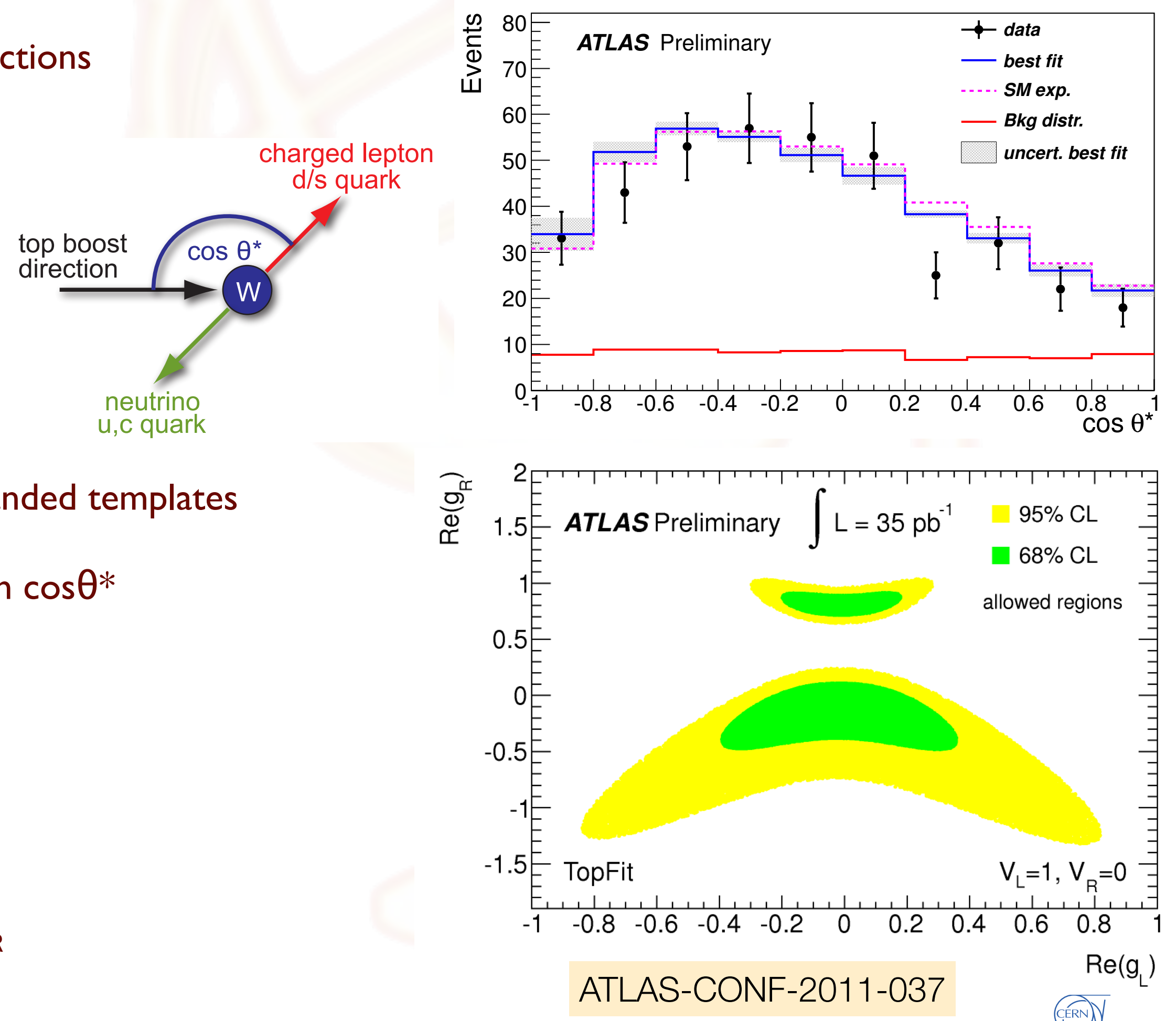


\section{Top pair production}

- Dilepton

- two cut-based analyses

- without and with b-tag

- 2 high-pt leptons (ee/ $\mu \mu / \mathrm{e} \mu), \geq 2$ jets

- ee/ $\mu \mu+$ jets: large missing $E_{T}$ and $Z \rightarrow \|$ veto

- e $\mu+$ jets: large $H_{T}$ (sum of jet and lepton PT)

- major backgrounds

- Z/Y* + jets, "fake" leptons

- (MC-assited) data-driven estimates

- Results

- combination of all dilepton channels

- profile likelihood

- no b-tagging

- $\sigma_{\overline{t t}}=173 \pm 22$ (stat.) ${ }^{+18}-16$ (syst.) ${ }^{+7}$-6 (lumi.) pb

- b-tagging

- $\sigma_{\overline{t t}}=|7| \pm 22$ (stat.) ${ }^{+2 \mid}{ }_{-16}$ (syst.) ${ }^{+7}{ }_{-6}$ (lumi.) pb

- Dominant uncertainties

- JES : $5 \%$

- parton shower model: $-5 \% /+5 \%$

- fake leptons : $-4 \% /+3 \%$

- Simultaneous measurement of b-tagging efficiency

- fit to number of b-tags

- consistent results

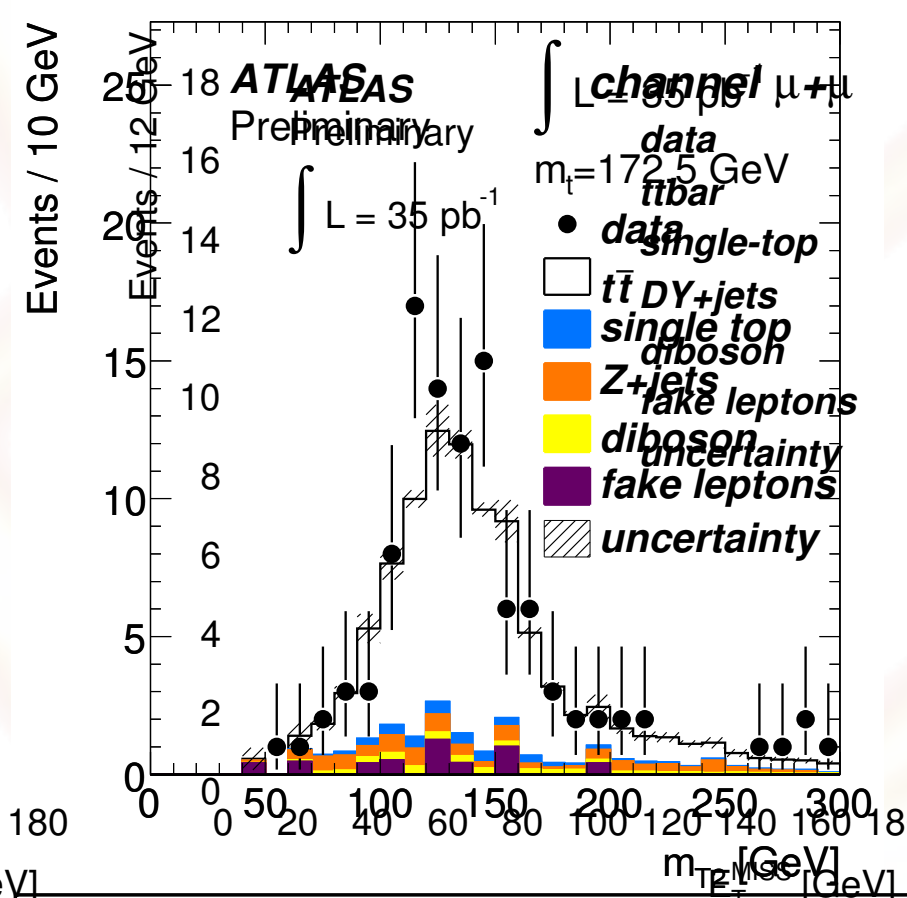

no b-tag: max. transverse mass
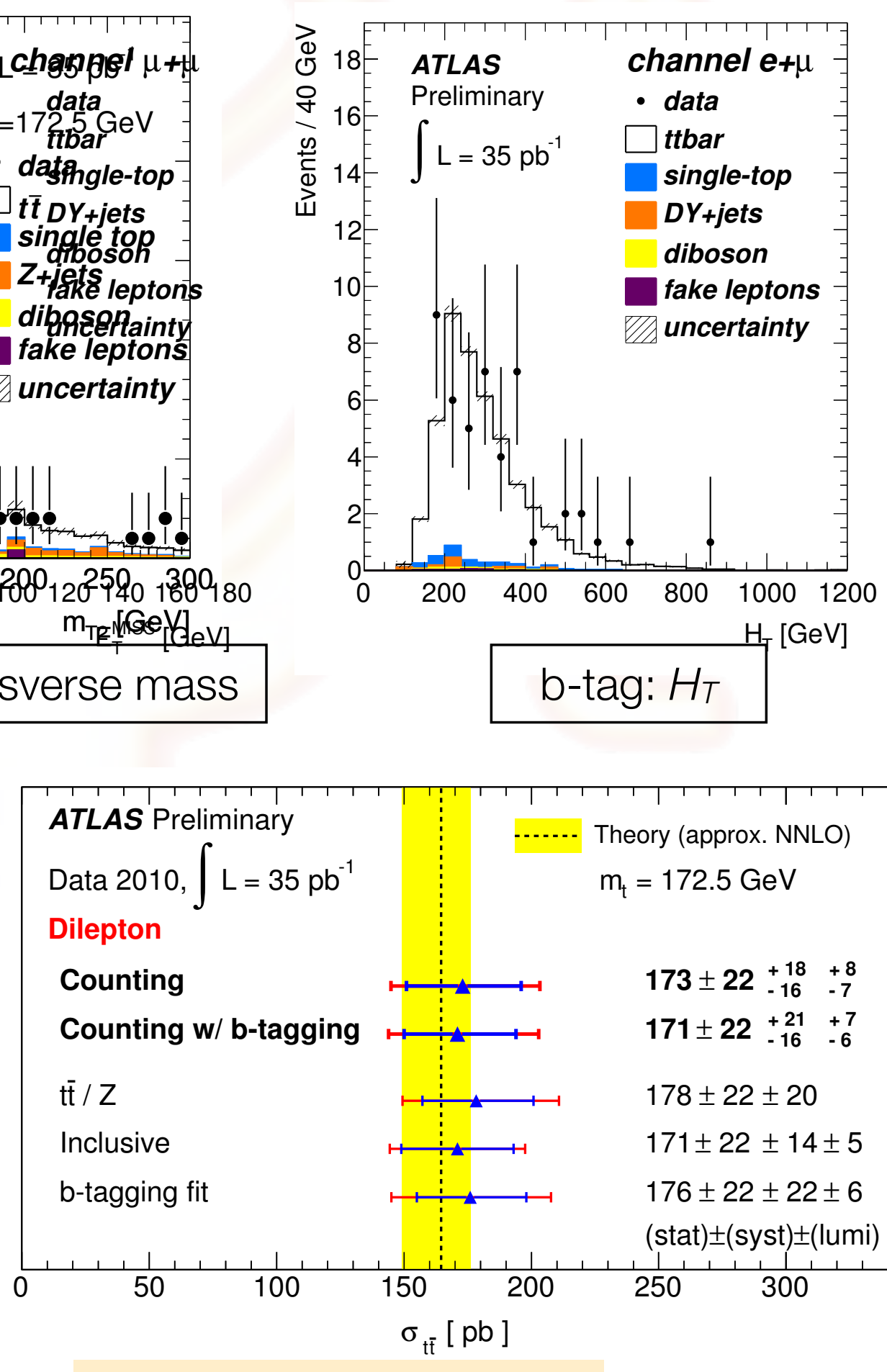

ATLAS-CONF-2011-034

Toshi SUMIDA

59

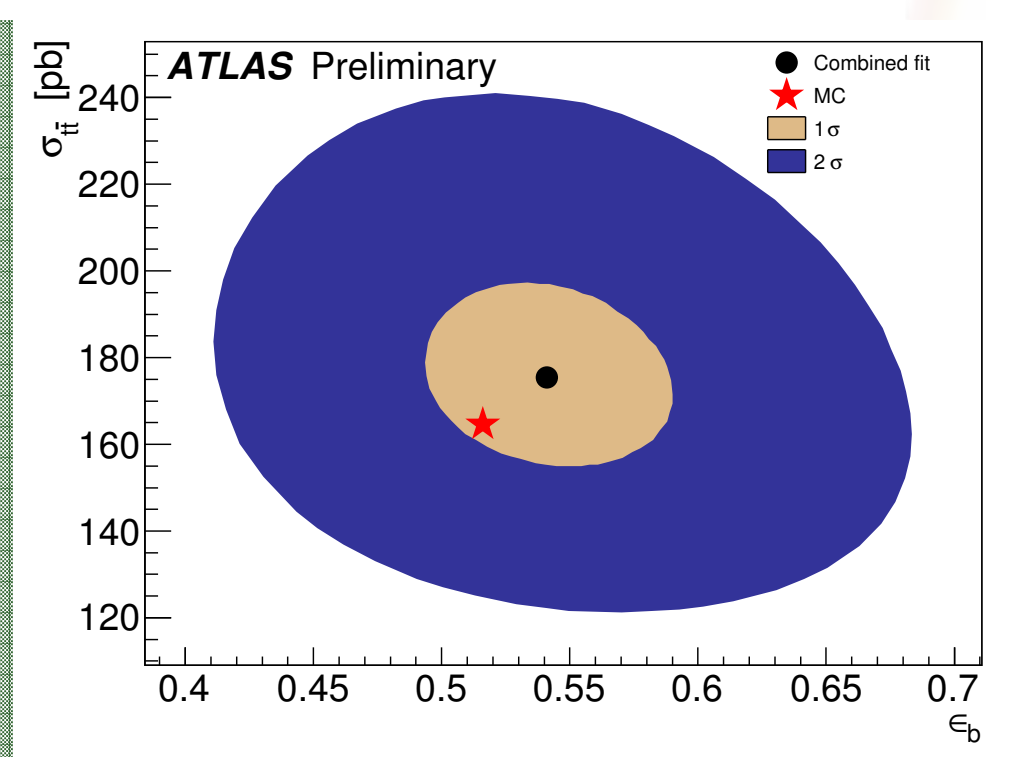

standard TModel results from AILAS 